\title{
Sequential Monte Carlo Particle Filtering for State Estimation
}

\author{
by \\ Laurence Smith \\ A thesis submitted to \\ The Faculty of Graduate Studies and Research \\ in partial fulfilment \\ of the requirements for the degree of \\ Master of Applied Science in Electrical Engineering
}

Ottawa-Carleton Institute for Electrical and Computer Engineering

Department of Systems and Computer Engineering

Carleton University

Ottawa, Ontario

August 29, 2006

(C) Copyright

2006, Laurence Smith 


$\begin{array}{ll}\begin{array}{l}\text { Library and } \\ \text { Archives Canada }\end{array} & \begin{array}{l}\text { Bibliothèque et } \\ \text { Archives Canada }\end{array} \\ \begin{array}{l}\text { Published Heritage } \\ \text { Branch }\end{array} & \begin{array}{l}\text { Direction du } \\ \text { Patrimoine de l'édition }\end{array} \\ \begin{array}{l}\text { 395 Wellington Street } \\ \text { Ottawa ON K1A ON4 }\end{array} & \begin{array}{l}\text { 395, rue Wellington } \\ \text { Ottawa ON K1A ON4 } \\ \text { Canada }\end{array}\end{array}$

Your file Votre référence ISBN: 978-0-494-18332-8 Our file Notre référence ISBN: 978-0-494-18332-8

NOTICE:

The author has granted a nonexclusive license allowing Library and Archives Canada to reproduce, publish, archive, preserve, conserve, communicate to the public by telecommunication or on the Internet, loan, distribute and sell theses worldwide, for commercial or noncommercial purposes, in microform, paper, electronic and/or any other formats.

The author retains copyright ownership and moral rights in this thesis. Neither the thesis nor substantial extracts from it may be printed or otherwise reproduced without the author's permission.
AVIS:

L'auteur a accordé une licence non exclusive permettant à la Bibliothèque et Archives Canada de reproduire, publier, archiver, sauvegarder, conserver, transmettre au public par télécommunication ou par l'Internet, prêter, distribuer et vendre des thèses partout dans le monde, à des fins commerciales ou autres, sur support microforme, papier, électronique et/ou autres formats.

L'auteur conserve la propriété du droit d'auteur et des droits moraux qui protège cette thèse. $\mathrm{Ni}$ la thèse ni des extraits substantiels de celle-ci ne doivent être imprimés ou autrement reproduits sans son autorisation.
In compliance with the Canadian

Privacy Act some supporting forms may have been removed from this thesis.

While these forms may be included in the document page count, their removal does not represent any loss of content from the thesis.
Conformément à la loi canadienne sur la protection de la vie privée, quelques formulaires secondaires ont été enlevés de cette thèse.

Bien que ces formulaires aient inclus dans la pagination, il n'y aura aucun contenu manquant.

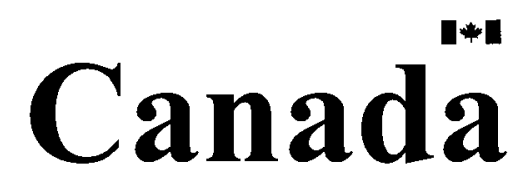




\section{Abstract}

State estimation is of paramount importance in many fields of Engineering including statistical signal processing, robot localization, and target tracking. Filtering is a way of estimating the state of a system by incorporating noisy observations as they become available online with prior knowledge of the system model. Particle filters are sequential Monte Carlo methods that use a point mass representation of probability densities in order to propagate the required statistical properties for state estimation.

This thesis quantitatively compares the generic and auxiliary particle filtering frameworks using various proposal densities and state characterizations. New particle filtering methods that use the extended and unscented Kalman filters in the auxiliary framework are introduced. All the methods are compared in terms accuracy and robustness.

Synthetic stochastic models that incorporate nonlinear, non-stationary, and nonGaussian elements are used for the experiments. The newly proposed auxiliary unscented Kalman particle filter is shown to outperform existing nonlinear filters in many of the experiments. 


\section{Acknowledgements}

Firstly, I would like to thank my thesis supervisor, Prof. Aitken, for all his help and support. His cheerful demeanour and optimistic outlook kept me motivated throughout the hard work. Furthermore, I would like to thank my parents for all their support both during my time here at Carleton and during my previous degree at UBC. Without their support none of this would have been possible. And finally, I would like to thank my girlfriend Roz. She too has helped me throughout most of my post-secondary life. I would like to point out that she has found more of my spelling mistakes and algebraic mishaps than anyone else. Simply, she makes my life better. 
The undersigned recommends to

The Faculty of Graduate Studies and Research acceptance of the thesis

\author{
Sequential Monte Carlo Particle Filtering \\ for State Estimation \\ submitted by \\ Laurence Smith \\ in partial fulfilment \\ of the requirements for the degree of \\ Master of Applied Science in Electrical Engineering
}

Thesis Supervisor

Chairperson, Department of Systems and Computer Engineering

Carleton University

August 28, 2006 


\section{Contents}

1 Introduction $\quad 1$

1.1 State Estimation . . . . . . . . . . . . . . . . 1

1.2 Motivation for Sequential Monte Carlo Methods . . . . . . . . . . . . 3

1.3 Thesis Statement . . . . . . . . . . . . . . 6

1.4 Thesis Overview . . . . . . . . . . . . . . . . 9

2 The Monte Carlo Method 11

2.1 From Lviv to Monte Carlo . . . . . . . . . . . . . . . . . . . . 11

2.2 Buffon's Needle . . . . . . . . . . . . . . . . . . . 13

2.3 Monte Carlo Integration . . . . . . . . . . . . . . . 15

2.4 Applications of the Monte Carlo Method . . . . . . . . . . . . . 17

2.5 Chapter Summary . . . . . . . . . . . . . . . 19

3 Filtering Theory $\quad 20$

3.1 Introduction . . . . . . . . . . . . . . . . 20

3.2 Classical Regression Theory . . . . . . . . . . . . . . 22

3.2.1 Recursive Least-Squares Estimation . . . . . . . . . . . . 27

3.3 The Kalman Filter . . . . . . . . . . . . . . . 30

3.3.1 Relating Recursive Bayesian Estimation and the Kalman Filter 32

3.3.2 General State-Space . . . . . . . . . . . . 34 
3.3.3 The Extended Kalman Filter . . . . . . . . . . . 36

3.3.4 Tuning the $\mathrm{R}$ and $\mathrm{Q}$ Parameters . . . . . . . . . . . 37

3.4 The Unscented Kalman Filter . . . . . . . . . . . . . . . . . . . 40

3.4.1 The Unscented Transform ... . . . . . . . . . . . 41

3.4.2 The Scaled Unscented Transform . . . . . . . . . . . . 43

3.4.3 Implementation of the Unscented Kalman Filter . . . . . . . . 45

3.5 Sequential Monte Carlo Methods (Particle Filters) . . . . . . . . . . . 48

3.5.1 Perfect Monte Carlo Simulation . . . . . . . . . . . . . . . . 49

3.5.2 Bayesian Importance Sampling . . . . . . . . . . . . 50

3.5.3 Sequential Importance Sampling . . . . . . . . . . . . . . 51

3.5.4 Resampling and the Generic Particle Filter . . . . . . . . . . 54

3.5.5 Sample Importance Resampling . . . . . . . . . . . . . . 57

3.5.6 Extended and Unscented Kalman Particle Filters . . . . . . 59

3.5.7 The Auxiliary Particle Filter . . . . . . . . . . . . 60

3.6 Chapter Summary . . . . . . . . . . . . . . . 62

4 Synthetic Nonlinear Estimation Problems $\quad 63$

4.1 Metrics for Comparison and Complexity . . . . . . . . . . 63

4.2 Gaussian Noise Model Results . . . . . . . . . . . . . . . . 64

4.2.1 Model Description ................ 64

4.2.2 Calculating the Likelihood and Prior Densities . . . . . . . . 65

4.2.3 Changing the Number of Particles ........... 65

4.2.4 Comparing the Results from the Three Observation Stages . . 67

4.2.5 Changing the Noise Variances ............. 72

4.3 Gamma Noise Model Results . . . . . . . . . . . . . . . . . 73

4.3.1 Model Description ................ 73 
4.3.2 Calculating the Likelihood and Prior Densities . . . . . . . . 74

4.3.3 Gamma Noise Results . . . . . . . . . . . . . . . 75

4.4 Cauchy Noise Model Results . . . . . . . . . . . . . . . . . 76

4.4.1 Model Description . . . . . . . . . . . . . 76

4.4.2 Calculating the Likelihood and Prior Densities . . . . . . . . 79

4.4.3 Cauchy Noise Results . . . . . . . . . . . . . . . . 80

4.4.4 Comparing Single Runs of the Cauchy Noise Simulations . . . 83

4.5 Chapter Summary . . . . . . . . . . . . . . . 85

5 Sample Applications: Simulation and Results $\quad 87$

5.1 Stochastic Volatility Model . . . . . . . . . . . . . . 87

5.1 .1 Introduction . . . . . . . . . . . . 87

5.1 .2 Model Description . . . . . . . . . . . . 88

5.1.3 Simulation and Results . . . . . . . . . . . . . . 88

5.2 2D Target Tracking With Range and Bearing Measurements . . . . 92

5.2 .1 Introduction . . . . . . . . . . . . . . 92

5.2 .2 Model Description ................. 92

5.2.3 Jacobian Calculations . . . . . . . . . . . . 95

5.2 .4 Initialization . . . . . . . . . . . . . . 96

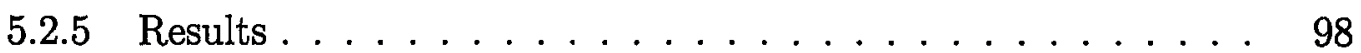

5.3 Chapter Summary . . . . . . . . . . . . . . . . 99

6 Conclusions and Recomendations 103

6.1 Conclusions ....................... 103

6.2 Contributions ...................... 107

6.3 Recommendations . . . . . . . . . . . . . . . 108 
A.1 Introduction to Random Variables . . . . . . . . . . . . . . . . . 119

A.1.1 Probability Space . . . . . . . . . . . . . . . . . . 119

A.1.2 Defining a Random Variable . . . . . . . . . . . . . . 120

A.1.3 Mathematical Expectation . . . . . . . . . . . . . . 123

A.1.4 Moments . . . . . . . . . . . . . . . . . 123

A.1.5 Joint Moments . . . . . . . . . . . . . . . . . . . 124

A.2 Conditional Probability . . . . . . . . . . . . . . . . . 125

A.2.1 Basic Relations of Conditional Probability . . . . . . . . 129

A.2.2 Conditional Functions . . . . . . . . . . . . 130

A.3 Stochastic Processes . . . . . . . . . . . . . . . . . . 131

A.3.1 Properties of Stochastic Processes . . . . . . . . . . . . . 134

A.3.2 Gaussian Processes . . . . . . . . . . . . . 136

A.3.3 Markov Processes . . . . . . . . . . . . . . 138

A.4 Basic Statistical Properties of Random Variables . . . . . . . . . . . 140

A.4.1 The Strong Law of Large Numbers . . . . . . . . . . . 140

A.4.2 The Central Limit Theorem . . . . . . . . . . . . . 141

B Generating Random Numbers 143

B.1 Introduction . . . . . . . . . . . . . . . . . . . . 143

B.2 Properties of Random Numbers $\ldots \ldots \ldots \ldots \ldots$

B.3 Key Issues in Generating Pseudo-Random Numbers . . . . . . . . . 144

B.4 Generating Uniformly Distributed Pseudo-Random Numbers . . . . 145

B.4.1 The Linear Congruential Method . . . . . . . . . . 145 
B.4.2 The Lagged Fibonacci Algorithm with the Shift Register Random Integer Scheme . . . . . . . . . . . . . . . 147

B.5 Random Variate Generation . . . . . . . . . . . . . . . . 148

B.5.1 The Ziggurat Method: Gaussian Distribution . . . . . . . . . 148

B.5.2 The Acceptance-Rejection Technique: Gamma Distribution . 149

B.5.3 Inverse Transform Technique: Cauchy Distribution . . . . . . 150 


\section{List of Figures}

1.1 State Estimation $\ldots \ldots \ldots \ldots \ldots \ldots \ldots \ldots \ldots$

1.2 Example of State Estimation in Practice . . . . . . . . . . . 3

2.1 Buffon's Needle experimental setup. . . . . . . . . . . . . 13

3.1 Hidden Markov Model . . . . . . . . . . . . . . . . . . . . 34

$3.2 \quad$ Kalman Filter Parameter Simulation, $R=0.1 \ldots \ldots \ldots \ldots$

3.3 Kalman Filter Parameter Simulation, $R=1 \ldots \ldots \ldots \ldots$

3.4 Kalman Filter Parameter Simulation, $R=0.01 \ldots \ldots \ldots \ldots$

3.5 Graphical Interpretation of Resampling . . . . . . . . . . 57

3.6 Generic Particle Filter Flow Diagram . . . . . . . . . . . 58

4.1 Sample Gaussian Distributions . . . . . . . . . . . . . 66

4.2 EKF and UKF sample runs with Gaussian noise. . . . . . . . 68

4.3 SIRPF and ASIRPF sample runs with Gaussian noise. . . . . . . 68

4.4 EKPF and UKPF sample runs with Gaussian noise. . . . . . . . 69

4.5 AEKPF and AUKPF sample runs with Gaussian noise. . . . . . . . 69

4.6 Sample Gamma Distributions . . . . . . . . . . . . . . 75

4.7 EKF and UKF sample runs with Gamma noise. . . . . . . . . 77

4.8 SIRPF and ASIRPF sample runs with Gamma noise. . . . . . . 77 
4.9 EKPF and UKPF sample runs with Gamma noise. . . . . . . . . . 78

4.10 AEKPF and AUKPF sample runs with Gamma noise. . . . . . . . 78

4.11 Sample Cauchy Distributions . . . . . . . . . . . . . . . 80

$4.12 \mathrm{AEKPF}$ and AUKPF sample runs with Cauchy noise. . . . . . . 83

4.13 AEKPF and AUKPF sample runs with Cauchy noise. . . . . . . . . 84

4.14 SIRPF and ASIRPF sample runs with Cauchy noise. . . . . . . . 85

5.1 EKF and UKF sample runs with the stochastic volatility model. . . . 90

5.2 SIR and ASIR sample runs with the stochastic volatility model. . . . 90

5.3 EKPF and UKPF sample runs with the stochastic volatility model. . 91

5.4 AEKPF and AUKPF sample runs with the stochastic volatility model. 91

5.5 Path of Destruction. . . . . . . . . . . . . . . 93

5.6 EKF based filter results for the 2D tracking problem. . . . . . . . . 101

5.7 UKF based filter results for the 2D tracking problem. . . . . . . . . 102

A.1 The Monte Hall Problem. . . . . . . . . . . . . . . . 126

A.2 Brownian Motion . . . . . . . . . . . . . . 133

A.3 A graphical interpretation of stationarity. . . . . . . . . . . 136

A.4 Almost Surely . . . . . . . . . . . . . . . . 141

B.1 An illustration of the Ziggurat method with $N=8 \ldots \ldots 149$ 


\section{List of Tables}

2.1 Results of Monte Carlo Integration $\ldots \ldots \ldots \ldots \ldots$

3.1 SIS Particle Filter Algorithm . . . . . . . . . . . . . . 53

3.2 Particle Filter Resampling Algorithm . . . . . . . . . . . . 56

3.3 Generic particle filter framework. . . . . . . . . . . . . . 56

3.4 SIR Particle Filter Algorithm _ . . . . . . . . . . . . . . 59

3.5 Auxiliary particle filter framework. . . . . . . . . . . . 61

4.1 RMSE and Variance results using 10, 100, and 1000 particles with the additive Gaussian noise model. . . . . . . . . . . . . . . 67

4.2 RMSE and Variance results using 10 and 100 particles with the additive Gaussian noise model. . . . . . . . . . . . . . . . . . . 71

4.3 RMSE results using 10 and 100 particles with various additive Gaussian

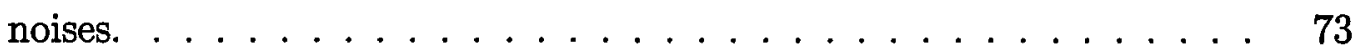

4.4 RMSE and Variance results using 10 and 100 particles with the additive Gamma noise model. . . . . . . . . . . . . . . . 76

4.5 RMSE and Variance results using 10 and 100 particles with the additive Cauchy noise model. . . . . . . . . . . . . . . . . . . . . . 81

4.6 RMSE and Variance results using 10 and 100 particles with the additive Cauchy noise model. . . . . . . . . . . . . . . . . . 82 
5.1 RMSE and Variance results using 10 and 100 particles with the stochastic volatility model. . . . . . . . . . . . . . . . . . . 89

5.2 RMSE and Variance results using 10 and 100 particles for the tracking

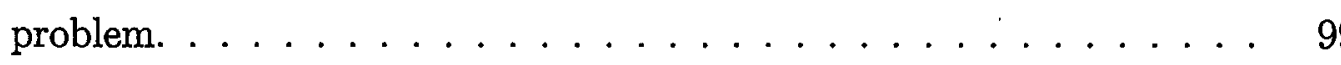




\section{Chapter 1}

\section{Introduction}

\section{$1.1 \quad$ State Estimation}

Control of complex systems such as aeronautical vehicles requires reliable real-time estimates of the system state. Similarly, much of the world's communication technology today involves the transmission of digital signals over noisy channels, and thus also requires reliable real-time estimates of the system state. For many complex systems, however, the complete state is not always directly measurable. Furthermore, in real world applications, often only noisy measurements of the system state are available. State estimation is the method of taking all the available information to determine the most likely state of a system at a point in time. State estimation techniques are useful tools that can track the gradual degradation of systems and their components, detect system faults, enable intelligent control, and predict future results.

State estimation can be divided into two main components: modeling and algorithms. To simulate the system, a model describing the underlying dynamics is needed. The system's behavior is often modelled mathematically as a set of stochastic differential equations. Analysis of the simulations conducted with system models 


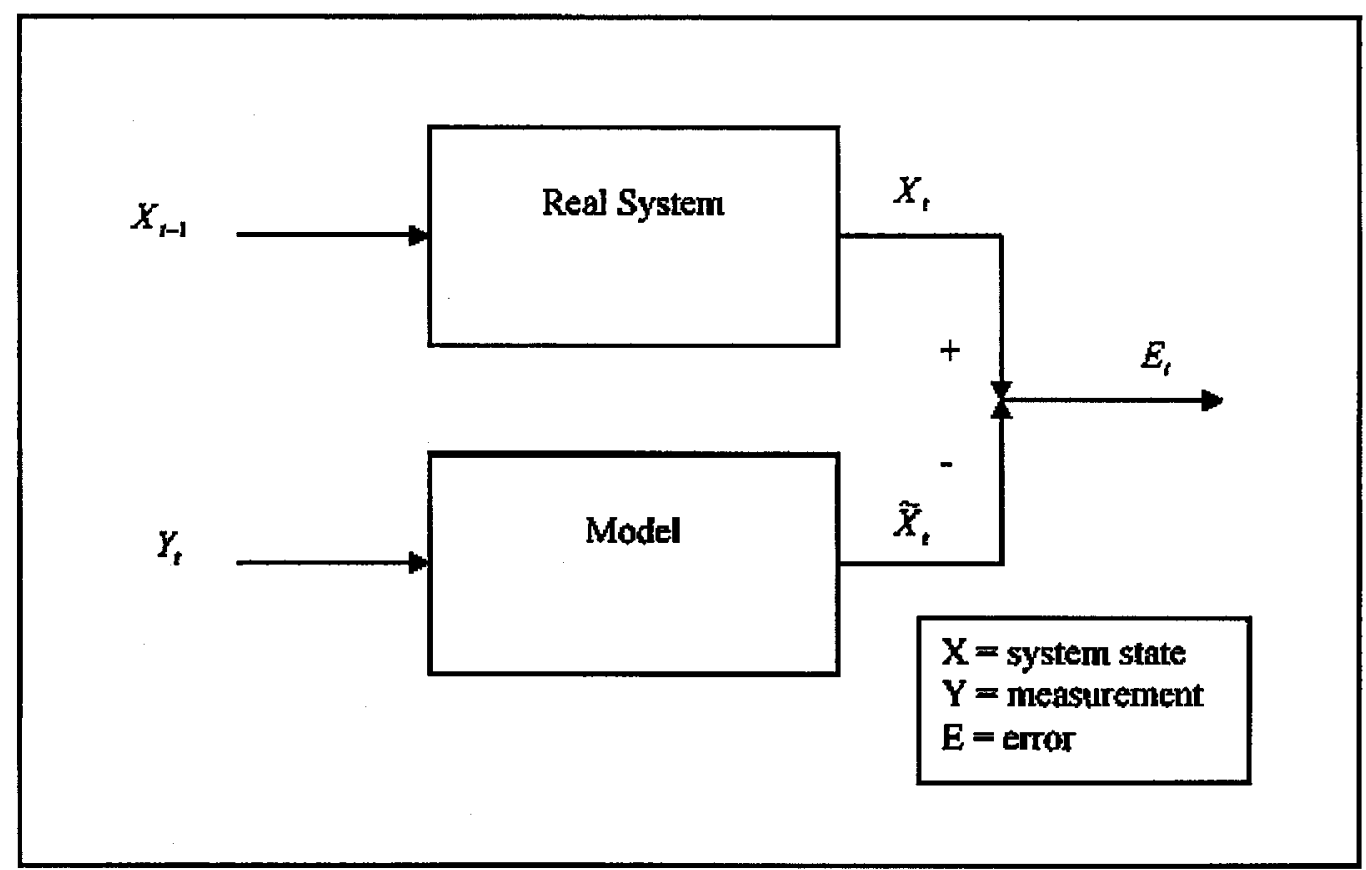

Figure 1.1: State Estimation - The goal of state estimation is to achieve an error equal to zero, i.e., $E=0$.

can help to determine which state or states lead to various system behavior. The results can be used to improve the design of the system. Once a system model is determined, a method to intelligently infer system states from the available data is required. Due to the complexity and nondeterministic nature of the systems, accurate models and efficient algorithms are needed for reliable state estimation. Refer to Figure 1.1 for an illustration of state estimation.

The goal of state estimation is to maintain an estimate of the system's states over some time period. For example, a system may be designed to monitor the altitude of an aircraft over the duration of a flight - see Figure 1.2. One approach to this problem is Bayesian belief updating. The state of the system is represented as a probability distribution that reflects the available state information. When new information is available through measurements the probability distribution is updated 


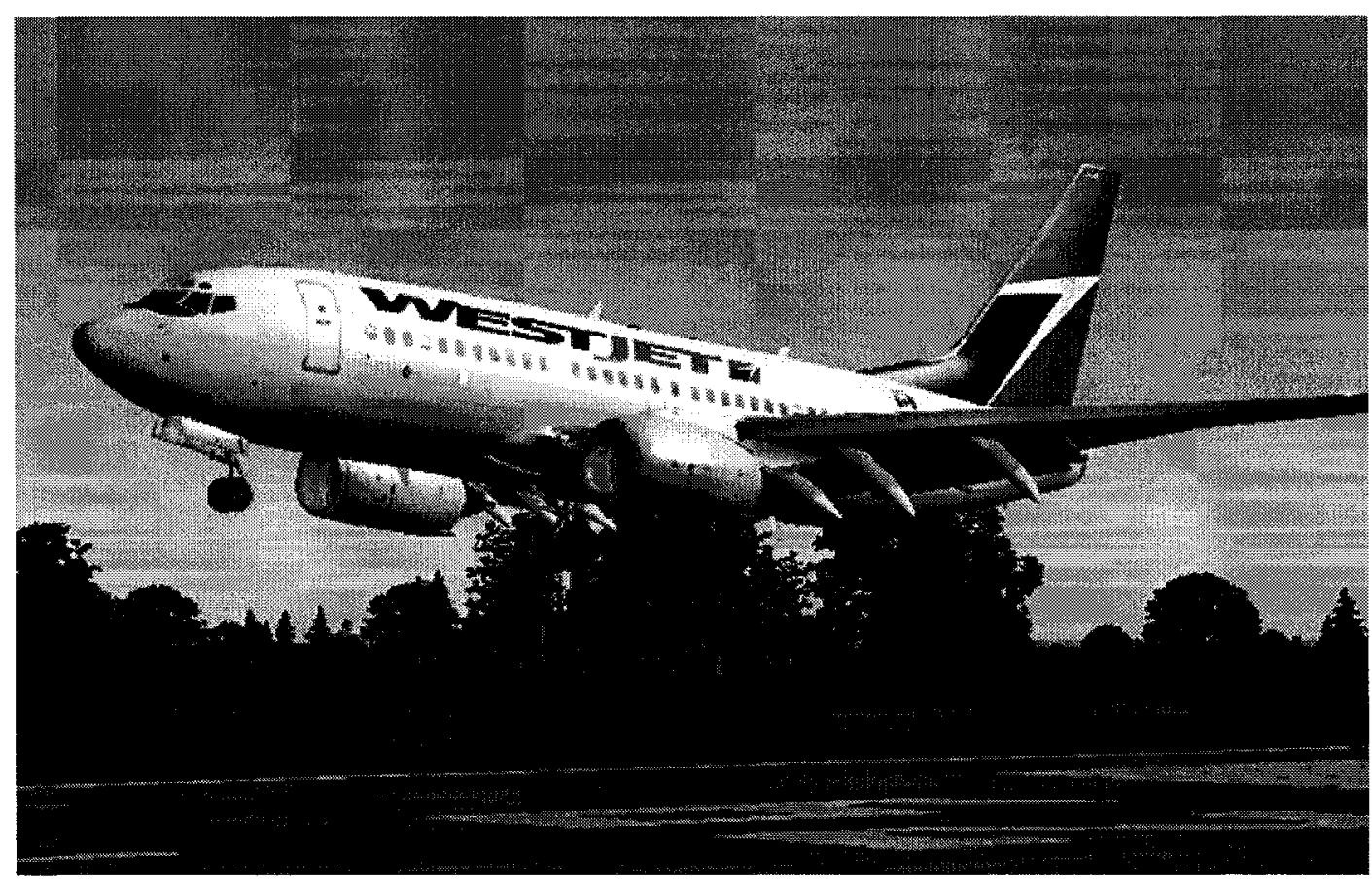

Figure 1.2: Example of State Estimation in Practice - Many sensors relay information to pilots (human pilots and computerized auto-pilots) during a flight about various flight variables including altitude. An intelligent scheme to determine an accurate estimation of the altitude based on the available measurement information is known as state estimation.

appropriately. Due to system resource constraints, exact computations are impossible and approximation schemes are used. Examples of approximations that are popular in scientific literature are the Extended Kalman Filter (EKF), the Unscented Kalman Filter (UKF), and Sequential Monte Carlo (SMC) methods.

\subsection{Motivation for Sequential Monte Carlo Meth- ods}

Many real-world applications of data analysis involve the estimation of unknown values, such as system states, from sets of observations. Prior knowledge of the 
system being modelled is often available allowing for the formulation of Bayesian models. Bayesian formulations include prior distributions for the unknown values and likelihood functions that relate the said values to observations. Using Bayes' theorem, a posterior density can be computed from the prior distributions and the likelihood functions

$$
\text { posterior }=\frac{\text { likelihood }}{\text { evidence }} \text { prior } \text {. }
$$

Inference of unknown quantities and their related statistics is then based on the resultant posterior density. Examples of such statistics are mean, mode, median, and confidence intervals. In reality, however, observations are usually collected sequentially in time and estimation of the unknown values is often required on-line. This motivates the idea of updating the posterior distribution as the observation data becomes available. Another consideration in real-world applications is computational simplicity required due to limited system resources. Storing all the observational data may not be necessary if the posterior distribution is updated sequentially in time.

There are two well known analytical solutions to sequentially update the posterior distributions. They are the Kalman filter and the hidden Markov model (HMM) filter. The Kalman filter can be used when the system is modelled as a linear Gaussian state-space model. Similarly, the HMM filter can be used when the system is modelled as a partially observed, finite state-space Markov chain. The simplifying assumptions mentioned above allow for analytically feasible solutions. In real-world applications, however, these assumptions are often not available as the system and observation models can be extremely complex. Real systems commonly include non-linear and non-Gaussian elements as well as high dimensionality. Numerical integration is another approach that could be used in non-linear, non-Gaussian cases. The problem with numerical integration is that it is computationally too expensive to be used in 
practical applications.

There are many practical applications with non-linear and non-Gaussian features, including, but not limited to: localization of robots, estimating noisy digital communications signals, chemical plant control, forecasting weather, estimating the volatility of financial instruments using stock market data, image processing, and aircraft tracking using radar measurements. These applications are all from a class of filtering problems which appear under of variety of names, including Bayesian filtering, optimal (non-linear) filtering, stochastic filtering, and on-line inference and learning [15].

Since Kalman's seminal work [33] on the optimal solution to linear/Gaussian state estimation problems in 1960, many approximation schemes have been derived to overcome the problems commonly encountered in filtering applications. Two of the more popular methods are the EKF and Gaussian sum approximations. In fact, the EKF is an extension on Kalman's work. The EKF is an approximation method for nonlinear state estimation problems that uses Taylor series expansion to estimate non-linearities in the system and observational models. Although they are used in practise quite often, EKF and Gaussian sum approximations fail to consider all the statistical information of the processes under consideration, and thus, can lead to poor results [15]. Increasingly, many applications require elements of both nonlinearity and non-Gaussianity for accurate models of the underlying physical dynamics. The UKF, an approximation based on the Unscented Transform, was recently developed in response to the new requirements and has proven to be superior to the EKF $[32,67]$. However, with recent advances in computational power, new methodologies for computing posterior distributions, namely, SMC methods, have become extremely popular $[3,4,9,11,15,20,22,26,36,37,39,49]$. SMC methods recursively approximate the integrals found in the Bayesian formulations for the posterior distributions 
via Monte-Carlo integration, hence, analytical solutions to the given problem are not needed.

Although the idea of Monte Carlo simulation originated in the late 1940's [53], its popularity in the field of filtering started in 1993 with the seminal publication, Novel approach to nonlinear/non-Gaussian Bayesian state estimation, by Gordon et al. [26]. The rejuvenation of the technique is due to the recent widespread availability of cheap computational power. A plethora of scientific papers from various research fields describing various SMC methods and applications has resulted. Variations of the SMC method are commonly found under several names, including: particle filters, bootstrap filters, Monte Carlo filters, condensation, and interacting particle approximations.

SMC methods are founded upon Monte Carlo simulations of the underlying systems and provide a convenient and attractive approach to computing the posterior distribution [15]. SMC methods are not limited by non-linearity and non-Guassianity constraints and can be implemented in a relatively simple fashion for a wide variety of problems. Due to the need for more complete physical models, the next generation of filtering methods will have to deal with nonlinear and non-Gaussian model components. SMC methods have the potential to use the increasing computational power available in todays technological market to push filtering theory beyond its next challenges.

\subsection{Thesis Statement}

The state-space approach to modelling dynamic systems has two main components. The first is a model describing the evolution of the state vector in time. Defining the 
state vector, $x_{k} \in \mathbb{R}^{n}$, the system model becomes,

$$
x_{k}=f_{k}\left(x_{k-1}, w_{k}\right)
$$

where $f_{k}: \mathbb{R}^{n} \times \mathbb{R}^{m} \rightarrow \mathbb{R}^{n}$ is the system transition function. The states represented by the state vector defined above are often referred to as hidden states since they are never actually observed directly. The system noise $w_{k} \in \mathbb{R}^{m}$ is usually modelled as a zero mean, white-noise sequence that is independant of past and current states.

The second is a model relating observations, $y_{k} \in \mathbb{R}^{p}$, to the state. This is accomplished with the measurement model,

$$
y_{k}=h_{k}\left(x_{k}, v_{v}\right)
$$

where $h_{k}: \mathbb{R}^{n} \times \mathbb{R}^{r} \rightarrow \mathbb{R}^{p}$ is the measurement function. Similar to the system noise, the measurement noise is usually a white-noise sequence independent of the previous and current states. The system and measurement noises are also usually independent of each other.

The probabilistic approach is then modelled as follows. The hidden states are modelled as a Markov process with initial distribution $p\left(x_{0}\right)$ and transition distributions $p\left(x_{k} \mid x_{k-1}\right)$ known as the priors. The observations $y_{k}$ are modelled with a marginal distribution $p\left(y_{k} \mid x_{k}\right)$ called the likelihood. Furthermore, we define the states and the observations up to time $k$ as $x_{0: k} \triangleq\left\{x_{0}, x_{1}, \ldots, x_{k}\right\}$ and $y_{1: k} \triangleq\left\{y_{1}, y_{2}, \ldots, y_{k}\right\}$ respectively.

The goal of SMC methods in relation to the aforementioned models is to recursively in time, $k$, estimate the posterior distribution $p\left(x_{0: k} \mid y_{1: k}\right)$ and ultimately the 
expectations

$$
\mathbb{E}_{p\left(x_{0: k} \mid y_{1: k}\right)}\left[\psi_{k}\left(x_{0: k}\right)\right] \triangleq \int \psi_{k}\left(x_{0: k}\right) p\left(x_{0: k} \mid y_{1: k}\right) d x_{0: k}
$$

for some function $\psi_{k}$. As an example, consider $\psi_{k}\left(x_{0: k}\right)=x_{0: k}$. The conditional mean, $\hat{x}_{k}$, of the system state is then estimated by

$$
\hat{x}_{k}=\mathbb{E}_{p\left(x_{0: k} \mid y_{1: k}\right)}\left[\psi_{k}\left(x_{0: k}\right)\right]=\int x_{0: k} p\left(x_{0: k} \mid y_{1: k}\right) d x_{0: k}
$$

By similar efforts, other statistics can also be estimated. For instance, Doucet et al. [15] suggest the conditional covariance of $\hat{x}_{k}$ where

$$
\psi_{k}\left(x_{0: k}\right)=x_{k} x_{k}^{T}-\mathbb{E}_{p\left(x_{k} \mid y_{1: k}\right)}\left[x_{k}\right] \mathbb{E}_{p\left(x_{k} \mid y_{1: k}\right)}^{T}\left[x_{k}\right]
$$

The objective of this thesis is to compare and analyze several algorithms recently proposed for consideration in scientific publications to estimate the expectation equation defined above. Contemporary SMC methods are compared with each other, the classical EKF method, and the recently realized UKF method. The effects of varying the SMC method's parameters as well as results of applying the methods to different system models is analysed. Furthermore, two new particle filters based on the EKF and UKF are proposed and tested. They are called the auxiliary extended Kalman particle filter (AEKPF) and the auxiliary unscented Kalman particle filter (AUKPF). Their results are also compared to those of the other filters. It is shown that in certain cases it can be advantageous to use the newly proposed filters. 


\subsection{Thesis Overview}

This chapter introduced the motivation behind using SMC methods for applications involving complex filtering problems. A brief history of filtering techniques was given. A description of the fundamental problem and contemporary solution methods were discussed.

The remainder of this thesis is organised as follows:

- Chapter 2 serves as an introduction to the Monte Carlo method and Monte Carlo integration. Monte Carlo integration is explained with Buffon's needle example. Recent applications of the Monte Carlo method are also discussed.

- Chapter 3 starts out explaining mean square estimation (MSE) and works towards more complicated estimation algorithms. The Kalman, extended Kalman, and unscented Kalman filters are explained, and finally an introduction to SMC estimation methods in terms of particles is given. This chapter lists the details of sequential importance sampling (SIS), sample importance Resampling (SIR), extended Kalman particle filters, and unscented Kalman particle filters. It also discusses the major issues involved in designing a successful SMC method. The two newly proposed partical filters, ie, the AEKPF and the AUKPF, are described near the end of this chapter. The tools from probability and stochastic processes necessary for the derivation of the SMC methods can be found in Appendix A.

- Chapter 4 describes synthetic experiments that the SMC methods were initially tested on. Both nonlinear and non-stationary model simulations are conducted. Models with Gaussian, Cauchy, and Gamma noise distributions are explored. The results of the SMC simulations are compared with each other 
and the classical methods. Analysis of the results when parameters of the models and the filters are varied is also presented.

- Chapter 5 illustates the results of two practical applications for the filtering methods. The first is an estimation problem involving the discrete time stochastic volatility model. This type of model is used in financial research to estimate asset volatility. The second simulation is of the $2 \mathrm{D}$ tracking problem. It is based on two ships moving at sea. A target ship is moving with a constant speed and bearing that is corrupted by noise. Meanwhile, a maneuvering observer ship with access to range and bearing data that is corrupted by noise tries to estimate the location of the target ship. This problem tests the various filters' abilities to estimate state vectors with multiple dimensions as opposed to the scalar cases presented in Chapter 4.

- Chapter 6 provides a concise listing of the conclusions and contributions of this thesis. It also recommends future work to further the particular research presented in this document. 


\section{Chapter 2}

\section{The Monte Carlo Method}

\subsection{From Lviv to Monte Carlo}

The Polish mathematician, Stanislaw Ulam, is routinely acknowledged as the inventor of the Monte Carlo method. Born in Lviv ${ }^{1}$ Galicja, Austria-Hungary ${ }^{2}$ Ulam was mentored by the great mathematician Stefan Banach at the Lwów School of Mathematics. In 1938 he moved to the United States as a Harvard Junior Fellow. Eventually he made his way to New Mexico to work at Los Alamos Laboratory. During World War II, Ulam worked on the United States' infamous Manhattan Project for John von Neumann. In particular, Ulam is most known for his work along side Edward Teller in 1951 on the hydrogen bomb. It was between these two events, in 1946, that Ulam thought up the Monte Carlo method while pondering the probabilities governing the game of solitaire. As quoted by Eckhardt [18]:

The first thoughts and attempts I made to practice [the Monte Carlo Method] were suggested by a question which occurred to me in 1946 as I was convalescing from an illness and playing solitaires. The question

\footnotetext{
${ }^{1}$ In Polish, Lwów.

${ }^{2}$ Now Ukraine.
} 
was what are the chances that a Canfield solitaire laid out with 52 cards will come out successfully? After spending a lot of time trying to estimate them by pure combinatorial calculations, I wondered whether a more practical method than abstract thinking might not be to lay it out say one hundred times and simply observe and count the number of successful plays. This was already possible to envisage with the beginning of the new era of fast computers, and I immediately thought of problems of neutron diffusion and other questions of mathematical physics, and more generally how to change processes described by certain differential equations into an equivalent form interpretable as a succession of random operations. Later [in 1946, I] described the idea to John von Neumann, and we began to plan actual calculations.

Although statistical sampling was well known in the mathematical arena, Von Neumann was intrigued by Ulam's idea of using the newly available electronic computing equipment to do the said sampling [18]. In particular, Von Neumann envisioned how valuable the approach could be if it were used to estimate and predict the explosive behavior the neutron chain reactions exploited in the fission weapons he was currently designing at Los Alamos Laboratory.

With the help of John von Neuman and Nicholas Metropolis, Ulam quickly developed algorithms for computer implementations along with ways to transform nonrandom problems into their equivalent random forms. By facilitating statistical sampling as a means to their respective solutions they effectively defined a formal methodology of solving problems that is applicable to many problems. Metropolis named the method after the casinos of Monte Carlo ${ }^{3}$. In 1948, Fermi, Metropolis and Ulam

\footnotetext{
${ }^{3} \mathrm{~A}$ town in Monaco located on the Mediterranean Sea and the French Riviera. It is world famous for its gambling-casinos and luxurious hotels.
} 


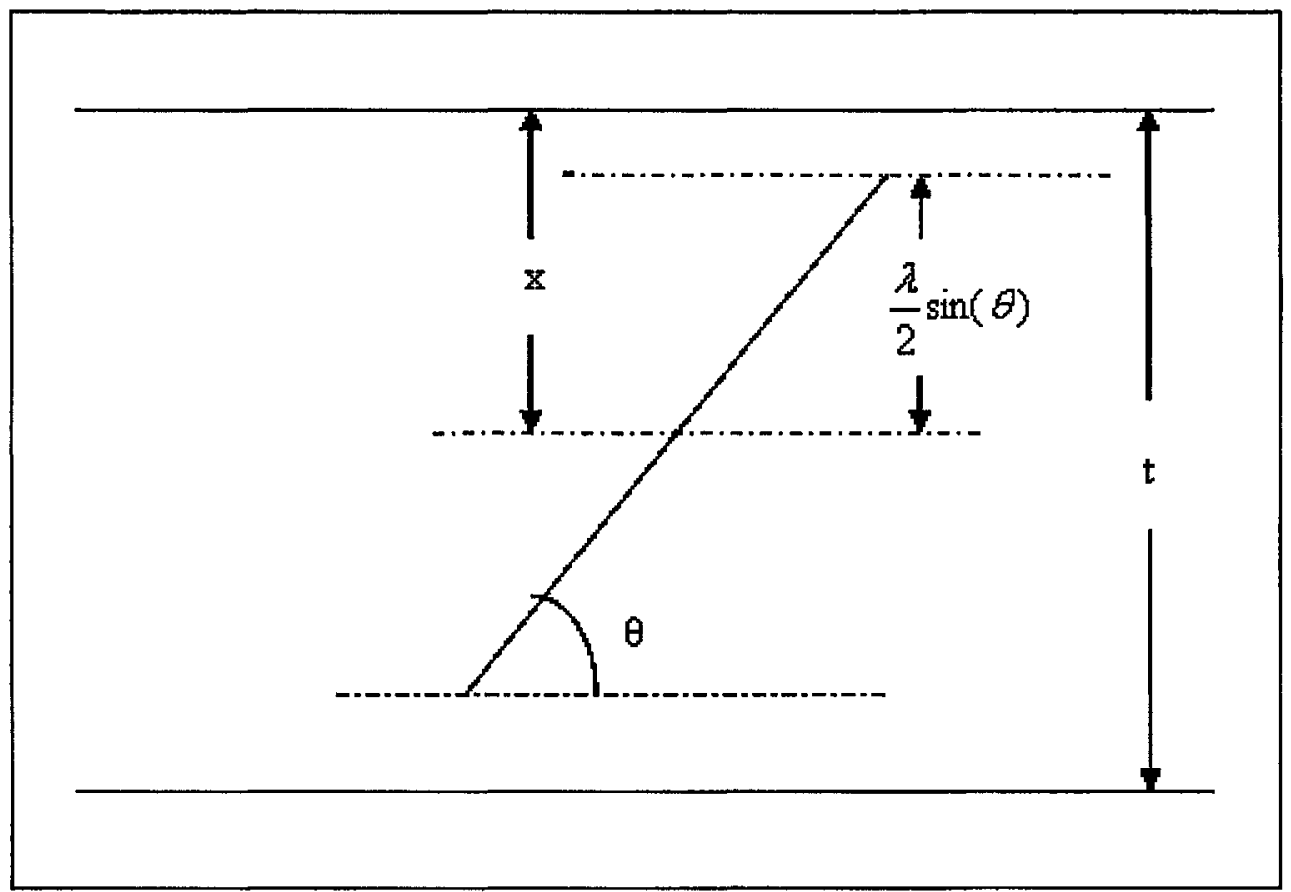

Figure 2.1: Buffon's Needle experimental setup.

calculated estimates for the eigenvalues of the Schrodinger equation with Monte Carlo techniques. Soon after, in 1949, Metropolis and Ulam published the first paper on the Monte Carlo method [53].

\subsection{Buffon's Needle}

As an introductory example to the workings of the Monte Carlo method, consider a problem proposed in the 18th century by Georges Leclerc, Comte de Buffon. Suppose a needle of length $\lambda$ is dropped on a floor made of parallel strips of wood, each with width $t$. What is the probability the needle ends up lying across a line between two strips of wood? Refer to Fig 2.1 for the experimental setup. By solving this problem with a Monte Carlo method, one can approximate the value for $\pi$.

From Fig 2.1 it is known that the distance from the middle of the needle to the 
closest line is $x$. The probability density of the random variable $x$ laying between 0 and $t / 2$ is

$$
p d f\left(0 \leq x \leq \frac{t}{2}\right)=\frac{2}{t} d x
$$

Similarly, the random variable that represents the acute angle between the needle and the lines, $\theta$, has a probability density function between 0 and $\frac{\pi}{2}$ that is

$$
\left.p d f\left(0 \leq \theta \leq \frac{\pi}{2}\right)\right)=\frac{2}{\pi} d \theta .
$$

Since $x$ and $\theta$ are independent random variables their joint probability density function is the product of their respective probability density functions. Also note that a needle crosses a line if

$$
x \leq \frac{\lambda}{2} \sin \theta
$$

Then the probability that a needle crosses a line can be found by integrating the joint probability density function:

$$
\begin{aligned}
P\left(x \leq \frac{\lambda}{2} \sin \theta\right) & =\int_{0}^{\frac{\pi}{2}} \int_{0}^{\frac{\lambda}{2} \sin \theta} \frac{4}{t \pi} d x d \theta \\
& =\int_{0}^{\frac{\pi}{2}} \frac{4}{t \pi} \frac{\lambda}{2} \sin \theta d \theta \\
& =\frac{2 \lambda}{t \pi}
\end{aligned}
$$

To conduct the experiment drop the needle on the floor $n$ times. Count how many times the dropped needle ends up lying across a line on the floor, $b$. The experimentally calculated probability is $\frac{b}{n}$. Thus a Monte Carlo approximation for $\pi$ can be 
found as:

$$
\pi=\frac{2 \lambda n}{t b}
$$

It is interesting to note that Leclerc came up with this idea more than 200 years before Metropolis contrived the term Monte Carlo method.

The expression Monte Carlo method is, in fact, very general. Any stochastic technique, that is, any technique that employs the use of random numbers, probability, and statistics to investigate problems, is usually referred to as a Monte Carlo method. As Hammersley and Handscomb [27] put it

Monte Carlo methods comprise that branch of experimental mathematics which is concerned with experiments on random numbers.

\subsection{Monte Carlo Integration}

Fundamentally, the Monte Carlo method is a general technique of numerical integration. For instance, the Monte Carlo method is used in Sec 2.2 to approximate the

definite integral in Eqn 2.4. The convenience at which Monte Carlo methods can be used to evaluate high dimensional integrals is one of the method's main advantages [28]. A more general multi-dimensional definite integral suitable for the Monte Carlo method is as follows:

$$
\begin{aligned}
\psi & =\int_{0}^{1} \int_{0}^{1} \cdots \int_{0}^{1} f\left(x_{1}, x_{2}, \ldots, x_{n}\right) d x_{1} d x_{2} \cdots d x_{n} \\
& =\int_{(0,1)^{n}} f(\vec{x}) d \vec{x}
\end{aligned}
$$


To approximate a solution of the above integral with the Monte Carlo method, a vector of random numbers uniformly distributed over the region of integration, $\vec{U}$, is introduced. A random variable is procured by applying the function $f$ to the vector $\vec{U}$. The uniform random variable has a probability density function, $\phi(\vec{x})$, and its expectation is:

$$
\begin{aligned}
E[f(\vec{U})] & =\int_{(0,1)^{n}} f(\vec{x}) \phi(\vec{x}) d \vec{x} \\
& =\int_{(0,1)^{n}} f(\vec{x}) d \vec{x},
\end{aligned}
$$

since $\phi(\vec{x})$ is equal to one over the interval of integration. Equating equations 2.6 and 2.7 the solution to the multi-dimensional definite integral can be realized:

$$
\psi=E[f(\vec{U})]
$$

Similarly, by employing the Strong Law of Large Numbers from A.4.1 an estimate of the solution can be calculated by taking $N$ samples from the random vector, $\left\{U^{[1]}, U^{[2]}, \ldots, U^{[N]}\right\}:$

$$
\hat{\psi}=\frac{1}{N} \sum_{j=1}^{N} f\left(U^{[j]}\right)
$$

According to the Strong Law of Large Numbers, $\hat{\psi}$ will almost surely converge to $\psi$ (see Appendix A for more details). Furthermore, as a consequence of the Central Limit Theorem, the sampling distribution of $\hat{\psi}$ tends to the normal distribution as $N \rightarrow \infty[28]$. As a result, approximate confidence limits can be given for the estimate $\hat{\psi}$. Eqn 2.9 is known as the crude Monte Carlo predictor [28]. The standard error of 
the crude Monte Carlo predictor is:

$$
\frac{\sigma}{\sqrt{N}}
$$

Analysis of the standard error formula leads to two important points. First, increasing the sample size of the Monte Carlo method decreases the standard error. For instance, to halve the standard error, the number of samples must be quadrupled. The second point is that the standard error of the Monte Carlo method does not depend on the dimension of the integral. This is a distinguishing advantage of the Monte Carlo method.

To test the performance of the Monte Carlo method, let us consider the following integral

$$
\psi_{x}=\int_{0}^{\pi} \sin \left(\cos \left(x^{\frac{3}{2}} \arctan \left(\frac{1}{4+\sqrt{x}}\right)\right)\right) d x .
$$

This integral is extremely hard to solve analytically. Using the Monte Carlo method, however, this integral can be solved very simply. Before completing the simulations we first need a solution to compare too. For this, the numerical integrator, NIntegrate[], in Mathematica was used. It is an adaptive algorithm that can solve integration problems numerically to a specified precision. For the integral in Eqn 2.3, NIntegrate[] returned 2.4090. The Monte Carlo method was implemented in Matlab. The results are shown in Table 2.1. Notice the effect of increasing the sample size of the Monte Carlo method. The error decreases as predicted.

\subsection{Applications of the Monte Carlo Method}

Monte Carlo methods have been applied to many fields ranging from nuclear physics to economics. The way in which they are applied varies tremendously from one 


\begin{tabular}{ccc}
$\mathrm{n}$ & $\hat{\psi}_{x}$ & $\mid$ error $\mid$ \\
\hline 10 & 2.3274 & 0.0816 \\
100 & 2.3914 & 0.0176 \\
1000 & 2.4020 & 0.0070 \\
10000 & 2.4072 & 0.0018 \\
100000 & 2.4084 & 0.0006 \\
1000000 & 2.4089 & 0.0001 \\
\hline
\end{tabular}

Table 2.1: Results of Monte Carlo Integration - The first column represents how many random numbers were used in the simulation. The second column lists the Monte Carlo approximation to the integral. The third column presents the absolute values of the method error.

field to the next. To classify an experiment to be of type Monte Carlo, however, usually it just needs to include the use of random numbers to examine a problem. That being said, there are two main types of problems that can be handled by Monte Carlo methods: probabilistic and deterministic [27]. In the probabilistic case, random numbers are chosen such that they simulate a physical process. Solutions are then inferred from the behaviour of the random numbers to answers questions about the original problem.

Hammersley and Handscomb [27] suggest the insect growth and nuclear reactor problems as examples of the probabilistic case. For instance, one wishes to analyze the growth of an insect population based on specific statistics such as survival and reproduction rate. By assigning random numbers to represent various vital statistics of the individuals in the population such as age at death and number of reproductions in the life time, one gets a random sample similar to that which could be collected from field work. Other examples include studies of nuclear reactors, where the fundamental particles of nuclear physics obey probabilistic rather than deterministic laws. The random motions of neutrons or any other particles can be simulated with random numbers, and thus nuclear reactors can be analyzed without the cost of physical construction or safety risk. 
The deterministic type of Monte Carlo experiment arises when the theoretical formulation of a deterministic problem can be derived, however, the solution can not easily be solved for by analytical means. This type of problem is deterministic by nature, yet, the solution can be found numerically via a Monte Carlo simulation involving random numbers. In this case, Hammersley and Handscomb point to problems in electromagnetic theory which require the solution to Laplace's equation subject to various boundary conditions that baffle standard analytical techniques.

In this thesis the Monte Carlo method is used in conjunction with Bayesian theory for the purpose of nonlinear filtering. Applications for this kind of algorithm cover a broad range of studies. For instance, in engineering SMC methods have been used for gesture recognization [7], fault detection in autonomous robots [12], mobile robot localization [22], highway traffic monitoring [40], ellipsometry measurements [49], and target tracking $[36,52,61,65]$. There have also been many other applications of the SMC methods in other fields such as mathematical finance $[9,20,29,45,58]$.

\subsection{Chapter Summary}

This chapter brought forward the Monte Carlo method, the main idea used in sequential Monte Carlo methods. This concept was illustrated with Buffon's needle example. A numerical example was also used to show the Monte Carlo Integration method. The main purpose of this introductory chapter (in conjunction with Appendix A) is to lay the theoretical foundations required in the subsequent chapters. 


\section{Chapter 3}

\section{Filtering Theory}

\subsection{Introduction}

The theory of estimation was initiated in the field of astronomical studies in which planet and comet orbits were analyzed using telescopic measurement data. There are six parameters that characterize the elliptical motion of planetary bodies. Using the telescopic measurement data, the goal was to estimate those parameters.

Today, it is accepted that least-squares regression originated with two people $[64,59]$. It was Legendre who coined the term Moindes Carrés or least squares in his 1805 publication Nouvelles Méthodes pour la Determination des Orbites des Comètes [46]. Gauss, on the other hand, used the least-squares method in 1795; however, he

did not publish his results until 1809 in his book Theoria Motus Corporum Celestium [24]. It is interesting to note that both of these distinguished scientists discovered the least-squares regression method independently while solving similar problems. It was in 1823, in a series of papers with the collective title Theoria Combinationis Observationum Erroribus Minimis Obnoxiae that Gauss published what is known today as the Gauss-Markov theorem [70]: 
...the best linear unbiased estimator of any linear combination of the coefficients is its least-squares estimator.

Until the early 20th century there were few developments in estimation theory after the least-squares method developed by Legendre and Gauss. In 1912, Fisher published his first work on the Maximum Likelihood Estimation method (MLE) [21]; however, it was not until 1921 that he coined the term Maximum Likelihood [19]. MLE is a parameter estimation routine used to determine the parameters that maximize the probability (or likelihood) of the sample data. In 1941 and 1942, Kolmorgorov [41] and Wiener [69] independently published papers on linear minimum mean-square error estimation. Their work - now referred to as Kolmogorov-Wiener filter theory laid the foundation on which Kalman built his famous discrete-time Kalman filter in 1960 [33]. A year later, in collaboration with Bucy, Kalman released another paper [34] describing the continuous-time Kalman filter.

With advances in computing technology, many practical control theory applications involving more elaborate algorithms were studied. The next hurdle became nonlinear filtering. This led Anderson and Moore to the extended Kalman filter in 1979 [2]. Just recently, in 1997, Juilier and Uhlmann [32] introduced the unscented Kalman filter. Both the extended and unscented Kalman filters are suboptimal versions of the original Kalman filter.

The huge advancement in computer power in the late 20th century has stimulated research in alternative approaches to the filtering problem. In 1993, Gordon, Salmond, and Smith introduced what is known as the bootstrap filter in their paper, Novel approach to nonlinear/non-Gaussian Bayesian state estimation [26]. This publication is commonly referred to as the seminal paper of the field of particle filtering. Particle filtering is a sequential Monte Carlo method of Bayesian analysis used for state estimation. Since then, various alterations and extensions to the bootstrap filter 
have been proposed and experimented with. The following is a list of some of the most significant particle filter advancements since 1993: quasi-Monte-Carlo particle filters [11, 20], the extended and unscented Kalman particle filters [66], the marginal particle filter [39], the auxiliary particle filter [58], the regularized particle filter [3], Gaussian sum particle filtering [43], and particle filters with Rao-Blackwellization [48].

The rest of this chapter is dedicated to introducing the mathematics behind some of the classical and contemporary filtering methods mentioned above.

\subsection{Classical Regression Theory}

This section uses the calculus of conditional expectations to derive a simple regression model. It is classical in the sense that a regression relationship is postulated and that the unknown parameters are regarded as fixed quantities. Sample moments will be used to obtain empirical estimators. The derivation methods discussed in this section and next are mostly from the work of Pollock [59].

Let us start by defining a pair of random vectors $\mathrm{x}$ and $\mathrm{y}$. We will say that this pair of random vectors has a well-defined joint probability density function $p(x, y)$. The best predictor of $\mathrm{y}$ given that $\mathrm{x}$ is unknown is the unconditional expectation

$$
\begin{aligned}
E[y] & =\int_{y} \int_{x} y p(x, y) d x d y \\
& =\int_{y} y p(y) d y .
\end{aligned}
$$

If $\mathrm{x}$ is known the information contained in $\mathrm{x}$ can be used to improve the predictor. 
The best predictor is then the conditional expectation of $\mathrm{y}$ given $\mathrm{x}$ denoted

$$
\begin{aligned}
E[y \mid x] & =\int_{y} y \frac{p(x, y)}{p(y)} d y \\
& =\int_{y} y p(y \mid x) d y .
\end{aligned}
$$

The main idea involved in regression is then to find a relationship between the statistics of $\mathrm{x}$ and $\mathrm{y}$ and the conditional expectation of $\mathrm{y}$ given $\mathrm{x}$.

Let us postulate that the conditional expectation of $\mathrm{y}$ given $\mathrm{x}$ is a simple linear function of $x$

$$
E[y \mid x]=A x+\beta
$$

This is known as a linear regression equation. We denote the error of predicting y from the conditional expectation $\epsilon=y-E[y \mid x]$. Therefore, for $\mathrm{y}$ we have

$$
\begin{aligned}
y & =E[y \mid x]+\epsilon \\
& =A x+\beta+\epsilon
\end{aligned}
$$

The objective is to relate the vector $\beta$ and the matrix $A$ to the moment terms $\operatorname{var}(\mathrm{x})$, $\operatorname{var}(\mathrm{y})$, and $\operatorname{cov}(\mathrm{y}, \mathrm{x})$. Estimations of the moments can be found in a straightforward manner from a set of observations. By doing this, we are finding a relationship between the statistics of $\mathrm{x}$ and $\mathrm{y}$ and the conditional expectation of $\mathrm{y}$ given $\mathrm{x}$.

We start off working with $E[y \mid x]$. Notice that the conditional expectation $E[y \mid x]$ can be converted to an unconditioned expectation by multiplying by the marginal 
density function of $\mathrm{x}$ and by integrating with respect to $\mathrm{x}$

$$
\begin{aligned}
E[E[y \mid x]] & =E\left[\int_{y} y \frac{p(x, y)}{p(x)} d y\right] \\
& =\int_{y} \int_{x} y p(y, x) d x d y \\
& =E[y] .
\end{aligned}
$$

Using this result it is easy to solve for $\beta$ in terms of the expectations of $\mathrm{x}$ and $\mathrm{y}$

$$
\beta=E[y]-A E[x] .
$$

Similarly, the joint moment $E\left[y x^{T}\right]$ can be obtained by multiplying $E[y \mid x]$ by $x^{T}$ and by the marginal density function of $\mathrm{x}$, and then by integrating with respect to $\mathrm{x}$. Recalling Eqn 3.3 we get

$$
\begin{aligned}
E\left[y x^{T}\right] & =\int_{x} E[y \mid x] x^{T} p(x) d x \\
& =\int_{x}(A x+\beta) x^{T} p(x) d x \\
& =\int_{x} A x x^{T} p(x) d x+\int_{x} \beta x^{T} p(x) d x \\
& =A E\left[x x^{T}\right]+\beta E\left[x^{T}\right] .
\end{aligned}
$$

Using the definition of $\operatorname{cov}(y, x)$ the matrix $A$ can be solved for in terms of the second- 
order moments

$$
\begin{aligned}
\operatorname{cov}(y, x) & =E\left[y x^{T}\right]-E[y] E\left[x^{T}\right] \\
& =\beta E\left[x^{T}\right]+A E\left[x x^{T}\right]-\beta E\left[x^{T}\right]-A E[x] E\left[x^{T}\right] \\
& =A\left(E\left[x x^{T}\right]-E[x] E\left[x^{T}\right]\right) \\
& =A \operatorname{var}(x) .
\end{aligned}
$$

Rearranging Eqn 3.8 we see the result

$$
A=\operatorname{cov}(y, x) \operatorname{var}(x)^{-1}
$$

Finally, by substituting the above result into Eqn 3.3 and rearranging we get

$$
\begin{aligned}
E[y \mid x] & =A x+\beta \\
& =A x+E[y]-A E[x] \\
& =E[y]+\operatorname{cov}(y, x) \operatorname{var}(x)^{-1}(x-E(x)) .
\end{aligned}
$$

Thus the result is a linear equation with coefficients relying solely on the statistics of $\mathrm{y}$ and $\mathrm{x}$.

The same result can be obtained using derivatives. This second method makes it very clear where the term minimum mean square error (MMSE) comes from. First, the mean square error is defined as

$$
E\left[\epsilon^{T} \epsilon\right]=E\left[(y-A x-\beta)^{T}(y-A x-\beta)\right] .
$$

The idea behind this method is then to minimize the mean square error by choosing the optimal values for $A$ and $\beta$ using derivatives. Starting with $\beta$, we minimize the 
error by setting zero equal to the following derivative

$$
\begin{aligned}
0 & =\frac{\partial}{\partial \beta}\left(E\left[(y-A x-\beta)^{T}(y-A x-\beta)\right]\right) \\
& =E[-2(y-A x-\beta)] \\
& =-2(E[y]-A E[x]-\beta) .
\end{aligned}
$$

By dividing both sides by -2 and solving for $\beta$,

$$
\beta=E[y]-A E[x]
$$

The matrix $A$ can be solved for in a similar effort. First, substitute for $\beta$ using Eqn 3.6

$$
E\left[\epsilon^{T} \epsilon\right]=E\left[(y-A x-E[y]+A E[x])^{T}(y-A x-E[y]+A E[x])\right] .
$$

Then, as before, set the derivative with respect to $A$ to zero

$$
\begin{aligned}
0 & =\frac{\partial}{\partial A}\left(E\left[(y-A x-E[y]+A E[x])^{T}(y-A x-E[y]+A E[x])\right]\right) \\
& =E\left[\frac{\partial}{\partial A}(y-A x-E[y]+A E[x])^{T}(y-A x-E[y]+A E[x])\right] \\
& =E\left[2(y-A x-E[y]+A E[x])(E[x]-x)^{T}\right] \\
& =-E\left[y x^{T}\right]+E[y] E\left[x^{T}\right]+A\left(E\left[x x^{T}\right]-E[x] E\left[x^{T}\right]\right) \\
& =-\operatorname{cov}(y, x)+\operatorname{Avar}(x)
\end{aligned}
$$

Solving for $A$,

$$
A=\operatorname{cov}(y, x) \operatorname{var}(x)^{-1} .
$$


By substituting $A$ and $\beta$ back into Eqn 3.3 the same aforementioned linear regression equation is realized

$$
E[y \mid x]=E[y]+\operatorname{cov}(y, x) \operatorname{var}(x)^{-1}(x-E(x)) .
$$

\subsubsection{Recursive Least-Squares Estimation}

The recursive least-squares estimation algorithm can be derived from the work presented in the last section. Let us start by considering the $t^{\text {th }}$ instance of the regression model

$$
y_{t}=\beta_{t}^{T} x_{t}+\epsilon_{t}
$$

In the above equation $y_{t}$ is a scalar, $\beta_{t}$ and $x_{t}$ are vectors, and the error terms $\epsilon_{t}$ are independent scalars with $E\left[\epsilon_{t}\right]=0$ and $\operatorname{var}\left(\epsilon_{t}\right)=\sigma^{2}$ for all $t$. The $t^{\text {th }}$ estimate of $\beta$ is denoted $\beta_{t}$ and for the recursion an initial estimate $\beta_{0}=E[\beta]$ is required. At this time we also introduce what is known as the dispersion matrix, $P_{t}$, which is the covariance matrix of the estimator. Its initial value is $P_{0}=D(\beta)$. Finally, the empirical information available at time $t$ is the set of all observations $Y_{t}=\left\{y_{1}, y_{2}, \ldots, y_{t}\right\}$.

To generate the recursive least-squares algorithm, we start with what is known as the information equation

$$
R_{t+1}=\frac{1}{\sigma^{2}} \sum_{i=1}^{t+1} x_{i} x_{i}^{T}
$$

and also with an auxiliary equation

$$
g_{t+1}=\frac{1}{\sigma^{2}} \sum_{i=1}^{t+1} x_{i} y_{i}
$$

The estimation of the vector $\beta_{t}$ at time $t$ can now be written in terms of $R$ and $g$ as 


$$
\beta_{t}=R_{t}^{-1} g_{t}
$$

The idea of recursion is to update the estimation of the $\beta$ vector whenever new measurements are available. Thus, the information and auxiliary equations can be written appropriately as

$$
R_{t}=R_{t-1}+\frac{1}{\sigma^{2}} x_{t} x_{t}^{T}
$$

and

$$
g_{t}=g_{t-1}+\frac{1}{\sigma^{2}} x_{t} y_{t}
$$

respectively. These representations show how updating can occur as measurements become available. Using Eqn 3.21, the $\beta$ vector can be written in terms of $R_{t}$ and $R_{t-1}$

$$
\begin{aligned}
\beta_{t} & =R_{t}^{-1} g_{t} \\
& =R_{t}^{-1}\left(g_{t-1}+\frac{1}{\sigma^{2}} x_{t} y_{t}\right) \\
& =R_{t}^{-1}\left(R_{t-1} \beta_{t-1}+\frac{1}{\sigma^{2}} x_{t} y_{t}\right) .
\end{aligned}
$$

Then, using Eqn 3.22, $\beta$ can be written in terms of only the most recent $R_{t}$

$$
\begin{aligned}
\beta_{t} & =R_{t}^{-1}\left(\left(R_{t}-\frac{1}{\sigma^{2}} x_{t} x_{t}^{T}\right) \beta_{t-1}+\frac{1}{\sigma^{2}} x_{t} y_{t}\right) \\
& =R_{t}^{-1}\left(R_{t} \beta_{t-1}-\frac{1}{\sigma^{2}} x_{t} x_{t}^{T} \beta_{t-1}+\frac{1}{\sigma^{2}} x_{t} y_{t}\right) \\
& =\beta_{t-1}+\frac{1}{\sigma^{2}} R_{t}^{-1} x_{t}\left(y_{t}-x_{t}^{T} \beta_{t-1}\right) \\
& =\beta_{t-1}+\frac{1}{\sigma^{2}} R_{t}^{-1} x_{t} e_{t}
\end{aligned}
$$


where $e_{t}$ is the prediction error.

The next step is to replace the computationally inconvenient $R^{-1}$ with another recursion. The dispersion matrix, $P_{t}$ is defined in terms of the information matrix $R_{t}$ as

$$
\begin{aligned}
P_{t} & =R_{t}^{-1} \\
& =\left(R_{t-1}+\frac{1}{\sigma^{2}} x_{t} x_{t}^{T}\right)^{-1}
\end{aligned}
$$

By applying the matrix inversion lemma and rearranging the terms, $P_{t}$ can be simplified

$$
\begin{aligned}
P_{t} & =R_{t-1}^{-1}-R_{t-1}^{-1} x_{t}\left(x_{t}^{T} R_{t-1}^{-1} x_{t}+\sigma^{2}\right)^{-1} x_{t}^{T} R_{t-1}^{-1} \\
& =P_{t-1}-P_{t-1} x_{t}\left(x_{t}^{T} P_{t-1} x_{t}+\sigma^{2}\right)^{-1} x_{t}^{T} P_{t-1} \\
& =P_{t-1}-P_{t-1} x_{t} s_{t}^{-1} x_{t}^{T} P_{t-1} \\
& =\left(I-P_{t-1} x_{t} s_{t}^{-1} x_{t}^{T}\right) P_{t-1} \\
& =\left(I-k_{t} x_{t}^{T}\right) P_{t-1}
\end{aligned}
$$

where $s_{t}$ is the error dispersion and $k_{t}$ is the filter gain. Using the aforementioned relation we get

$$
\begin{aligned}
R_{t}^{-1} x_{t} & =P_{t} x_{t} \\
& =\left(P_{t-1}-P_{t-1} x_{t} s_{t}^{-1} x_{t}^{T} P_{t-1}\right) x_{t} \\
& =P_{t-1} x_{t} s_{t} s_{t}^{-1}-P_{t-1} x_{t} s_{t}^{-1} x_{t}^{T} P_{t-1} x_{t} \\
& =P_{t-1} x_{t}\left(x_{t}^{T} P_{t-1} x_{t}+\sigma^{2}\right) s^{-1}-P_{t-1} x_{t} s^{-1} x_{t}^{T} P_{t-1} x_{t} \\
& =\sigma^{2} P_{t-1} x_{t} s_{t}^{-1}
\end{aligned}
$$


Hence, the recursion equation for the $\beta$ vector is then

$$
\begin{aligned}
\beta_{t} & =\beta_{t-1}+\frac{1}{\sigma^{2}} R_{t}^{-1} x_{t} e_{t} \\
& =\beta_{t-1}+P_{t} x_{t} s_{t}^{-1} e_{t} \\
& =\beta_{t-1}+k_{t} e_{t}
\end{aligned}
$$

It is useful to list out the equations involved in the recursive least-squares algorithm for future reference. The list is as follows:

$$
\begin{array}{ll}
e_{t}=y_{t}-x_{t}^{T} \beta_{t-1} & \text { Prediction Error } \\
s_{t}=x_{t}^{T} P_{t-1} x_{t}+\sigma^{2} & \text { Error Dispersion } \\
k_{t}=P_{t-1} x_{t} s_{t}^{-1} & \text { Filter Gain } \\
\beta_{t}=\beta_{t-1}+k_{t} e_{t} & \text { Parameter Estimate } \\
P_{t}=\left(I-k_{t} x_{t}^{T}\right) P_{t-1} & \text { Estimate Dispersion. }
\end{array}
$$

For more information on recursive least-squares see [5].

\subsection{The Kalman Filter}

The Kalman filter was first developed in the 1960 s by R. E. Kalman. The algorithm is well known and there is much literature describing a plethora of experiments involving the filter. Most of the material found in this section and the next section is a summary from the sources $[1,25,30,33,34,59]$, however, there are many more sources available.

The Kalman filter is based upon a state-space model. The state-space model represents the system of interest. The Kalman filter uses observations available from the system along with the system model in order make estimations of the current 
system state. The state-space model is as follows

$$
\begin{array}{cc}
x_{t}=f_{t} x_{t-1}+\nu_{t}, & \text { TransitionEquation } \\
y_{t}=h_{t} x_{t}+\eta_{t}, & \text { ObservationEquation }
\end{array}
$$

where $x_{t}$ is the state vector and $y_{t}$ is the system observation. The error terms, $\nu_{t}$ and $\eta_{t}$, are the state disturbance and observation error. They are uncorrelated random vectors with zero mean such that

$$
\nu_{t} \sim N\left(0, Q_{t}\right) \quad \text { and } \quad \eta_{t} \sim N\left(0, R_{t}\right)
$$

where the notation $N(\alpha, \beta)$ respresents a Gaussian distribution with a mean of $\alpha$ and a variance of $\beta$. The observation equation is analogous to the regression equation, Eqn 3.18, in the prior section discussing the recursive least-squares algorithm.

When implementing the Kalman filter it is assumed that the matrices $f_{t}, h_{t}, Q_{t}$, and $R_{t}$ are known for all $t=1, \ldots, n$. Also, that there are initial estimates $x_{0}$ and $D\left(x_{0}\right)$ available for the state vector $x_{0}$ and the dispersion matrix $P_{0}$ respectively at time $t=0$. Furthermore, as in the recursive least-squares case, the empirical information available at time $t$ when using the Kalman filter is the set of observations $Y_{t}=\left\{y_{1}, y_{2}, \ldots, y_{t}\right\}$.

The Kalman filter is a set of equations very similar to that of the recursive leastsquares algorithm. The main difference is the addition of the state-vector estimates $x_{t \mid t-1}$ and $x_{t}$ and their corresponding dispersion matrices $P_{t \mid t-1}$ and $P_{t}$. They are present due to the transition equation, Eqn 3.31. Note that if $f=I$ and $Q_{t}=0$ the transition equation does not affect the filter. The Kalman filter equations are summarized below: 


$$
\begin{array}{ll}
x_{t \mid t-1}=f_{t} x_{t-1} & \text { State Prediction } \\
P_{t \mid t-1}=f_{t} P_{t-1} f_{t}^{T}+Q_{t} & \text { Prediction Dispersion } \\
e_{t}=y_{t}-h_{t} x_{t \mid t-1} & \text { Prediction Error } \\
S_{t}=h_{t} P_{t \mid t-1} h_{t}^{T}+R_{t} & \text { Error Dispersion } \\
K_{t}=P_{t \mid t-1} h_{t}^{T} S_{t}^{-1} & \text { Filter Gain } \\
x_{t}=x_{t \mid t-1}+K_{t} e_{t} & \text { State Estimate } \\
P_{t}=\left(I-K_{t} h_{t}\right) P_{t \mid t-1} & \text { Estimate Dispersion. }
\end{array}
$$

\subsubsection{Relating Recursive Bayesian Estimation and the Kalman Filter}

Looking at the Kalman filter equations from a probabilistic point of view we get what is known as recursive Bayesian estimation. We assume that the true state is an unobserved Markov process and that the measurements are observed states of a hidden Markov model. This is illustrated in Fig 3.1. Given the immediately previous state, the true state can be written independent of all earlier states since it is assumed to be Markovian

$$
p\left(x_{i} \mid x_{0}, \ldots, x_{i-1}\right)=p\left(x_{i} \mid x_{i-1}\right)
$$

Because of the Markov assumption the measurement is only dependent on the current true state

$$
p\left(y_{i} \mid x_{0}, \ldots, x_{i}\right)=p\left(y_{i} \mid x_{i}\right)
$$


Using the formulas above, the probability distribution over all the states of the hidden Markov model can be be simplified to

$$
\begin{aligned}
p\left(x_{0}, \ldots, x_{i}, y_{1}, \ldots, y_{i}\right)= & p\left(y_{i} \mid x_{i}, \ldots, x_{0}, y_{1}, \ldots, y_{i-1}\right) \\
& p\left(x_{i} \mid x_{i-1}, \ldots, x_{0}, y_{1}, \ldots, y_{i-1}\right) p\left(x_{i-1}, \ldots, x_{0}, y_{1}, \ldots, y_{i-1}\right) \\
= & p\left(y_{i} \mid x_{i}\right) p\left(x_{i} \mid x_{i-1}\right) p\left(x_{i-1}, \ldots, x_{0}, y_{1}, \ldots, y_{i-1}\right) \\
= & p\left(x_{0}\right) \prod_{i=1}^{i} p\left(y_{i} \mid x_{i}\right) p\left(x_{i} \mid x_{i-1}\right)
\end{aligned}
$$

The Kalman filter, however, is used to estimate the state $x_{i}$ and thus the probability distribution of interest is that of the current state conditioned on the most current measurement. There are two stages to the Kalman filter. The first stage predicts the true state based on the previous measurement. The second stage updates the prediction when the most current measurement is available. The prediction stage is realized using the Chapman-Kolmogorov relation

$$
p\left(x_{i} \mid Y_{i-1}\right)=\int p\left(x_{i} \mid x_{i-1}\right) p\left(x_{i-1} \mid Y_{i-1}\right) d x_{i-1}
$$

where $Y_{i}=\left\{y_{i}, \ldots, y_{0}\right\}$. The update stage can then be derived using Bayes' Rule

$$
p\left(x_{i} \mid Y_{i}\right)=\frac{p\left(y_{i} \mid x_{i}\right) p\left(x_{i} \mid Y_{i-1}\right)}{p\left(y_{i} \mid Y_{i-1}\right)}
$$

In the update stage, $p\left(y_{i} \mid x_{i}\right)$ is known as the measurement likelihood while $p\left(y_{i} \mid Y_{i-1}\right)$ is the normalizing factor. Using the Chapman-Kolmogorov relation again, the normalizing factor can be written

$$
p\left(y_{i} \mid Y_{i-1}\right)=\int p\left(y_{i} \mid x_{i}\right) p\left(x_{i} \mid Y_{i-1}\right) d x_{i}
$$




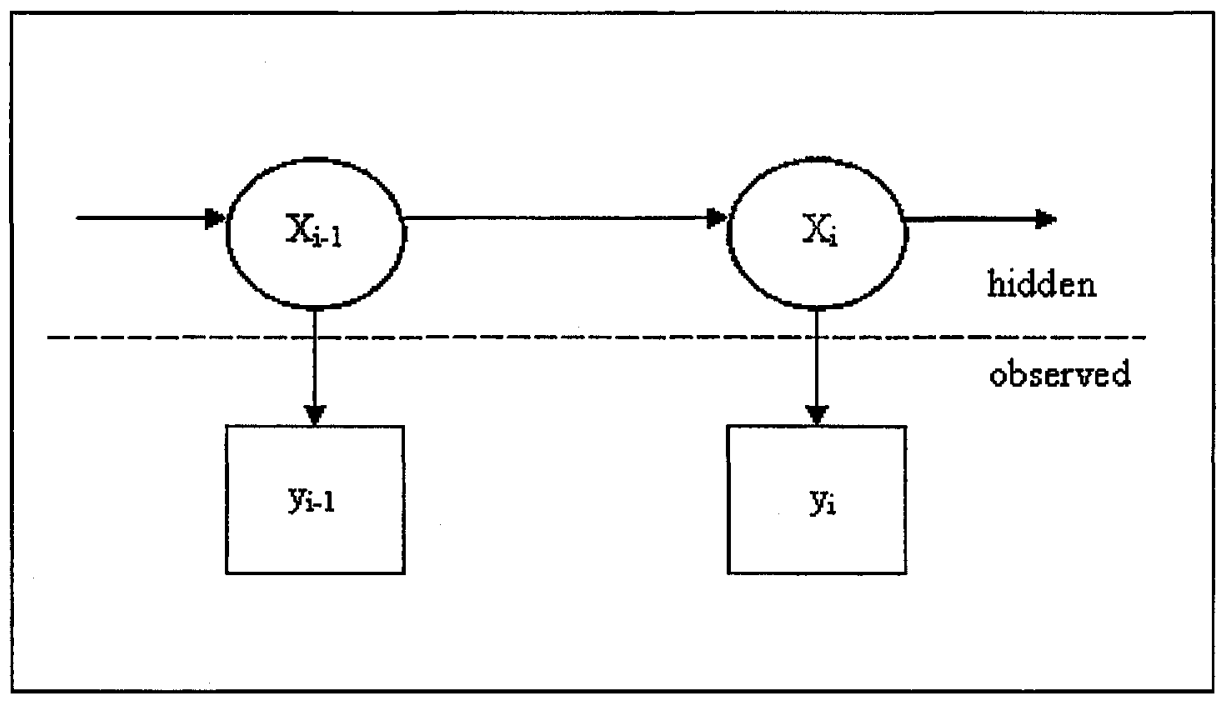

Figure 3.1: Hidden Markov Model - The true states are represented by $x$ while the observed states are represented by $y$.

Since the noise of the process and observation models is Gaussian, the remaining probability densities are

$$
\begin{gathered}
p\left(x_{i} \mid x_{i-1}\right)=N\left(f x_{i-1}, Q_{i-1}\right) \\
p\left(y_{i} \mid x_{i}\right)=N\left(h x_{i}, R_{i-1}\right) \\
p\left(x_{i-1} \mid Y_{i-1}\right)=N\left(\hat{x}, P_{i-1}\right) .
\end{gathered}
$$

The pdf for $x_{i}$ given $Y_{i}$ is the Kalman filter estimate.

\subsubsection{General State-Space}

In the previous section we derived the Kalman filtering routine for the state estimation of a linear system model. In practise, we would like to be able to remove the restriction that the transition and observation models be linear. Thus, in this section we introduce a general state-space model that can act as the framework for a more 
general discussion. The new form for the transition and observation models is

$$
\begin{gathered}
p\left(x_{t} \mid x_{t-1}\right) \\
p\left(y_{t} \mid x_{t}\right)
\end{gathered}
$$

where $x_{t} \in \mathbb{R}^{n_{x}}$ is the state vector at time $t$ and $y_{t} \in \mathbb{R}^{n_{y}}$ is the observation vector at time $t$. The nature of the states is a first order Markov process. Furthermore, the observations are assumed to be independent given the states.

Eqns 3.44 and 3.45 can be written in the notation similar to that of the previous section

$$
\begin{array}{r}
x_{t}=f\left(x_{t-1}, \nu_{t-1}\right) \\
y_{t}=h\left(x_{t}, \eta_{t-1}\right)
\end{array}
$$

where, again, $x_{t} \in \mathbb{R}^{n_{x}}$ is the state vector at time $t$ and $y_{t} \in \mathbb{R}^{n_{y}}$ is the observation vector at time $t$. In this case the process noise $\nu_{t} \in \mathbb{R}^{n_{\nu}}$ and the observation noise $\eta_{t} \in \mathbb{R}^{n_{\eta}}$ are independent zero mean processes with variances $Q$ and $R$ respectively. The system consists of two mappings. The first, $f: \mathbb{R}^{n_{x}} \times \mathbb{R}^{n_{\nu}} \mapsto \mathbb{R}^{n_{x}}$, represents the transition process. The second, $h: \mathbb{R}^{n_{x}} \times \mathbb{R}^{n_{\eta}} \mapsto \mathbb{R}^{n_{y}}$, represents the observation model.

The complete solution to the sequential estimation problem is the posterior density denoted $p\left(x_{0: t} \mid y_{1: t}\right)$ where $x_{0: t}=\left\{x_{0}, x_{1}, \ldots, x_{t}\right\}$ and $y_{1: t}=\left\{y_{1}, y_{2}, \ldots, y_{t}\right\}$. Keeping track of the posterior density may require too many system resources in many applications so alternatively it is often of interest to only store the filter density $p\left(x_{t} \mid y_{1: t}\right)$. Clearly the filtering density requires less memory, but furthermore, it can also be used to calculate various estimations of the system's states. 
The next sections introduce the extended Kalman filter and the unscented Kalman filter. They are two such approximation methods used to recursively solve the aforementioned filtering problem.

\subsubsection{The Extended Kalman Filter}

The Kalman filter has been shown to work well for linear systems, however, now we turn our attention to the nonlinear case. The EKF [2] is a minimum mean-square error (MMSE) estimator that is perhaps the most well known algorithm for solving the nonlinear filtering problem. It is similar to the Kalman filter except that it uses Taylor series expansions of the nonlinear system and observation functions, $f$ and $h$, around the estimates $\hat{x}_{t \mid t-1}$ of the states $x_{t}$ in order to facilitate the estimation of nonlinear systems.

The Jacobian of the system model is defined as

$$
\left.F_{t} \triangleq \frac{\partial f\left(x_{t}\right)}{\partial x_{t}}\right|_{\left(x_{t}=\hat{x}_{t-1}\right)}
$$

and similarly, the Jacobian of the observation model is

$$
\left.H_{t} \triangleq \frac{\partial h\left(x_{t}\right)}{\partial x_{t}}\right|_{\left(x_{t}=\hat{x}_{t / t-1}\right)}
$$

These are the linear expansion terms from the respective Taylor series. The mean $x$ and covariance $P$ for the Gaussian approximation to the posterior distribution of the states can then be determined with a method similar to the Kalman filtering technique. The extended Kalman filter is the following set of equations: 


$$
\begin{array}{ll}
\hat{x}_{t / t-1}=f\left(x_{t-1}, 0\right) & \text { State Prediction } \\
P_{t / t-1}=F_{t} P_{t-1} F_{t}^{T}+Q_{t} & \text { Prediction Dispersion } \\
e_{t}=y_{t}-h\left(\hat{x}_{t / t-1}, 0\right) & \text { Prediction Error } \\
S_{t}=H_{t} P_{t / t-1} H_{t}^{T}+R_{t} & \text { Error Dispersion } \\
K_{t}=P_{t / t-1} H_{t}^{T} S_{t}^{-1} & \text { Filter Gain } \\
\hat{x}_{t}=\hat{x}_{t / t-1}+K_{t} e_{t} & \text { State Estimate } \\
P_{t}=\left(I-K_{t} H_{t}\right) P_{t / t-1} & \text { Estimate Dispersion. }
\end{array}
$$

The EKF uses only the first term of the Taylor series expansion of the nonlinear functions. In cases where the system models are highly nonlinear the local linearity assumptions break down and the effects of the higher order Taylor expansion terms becomes evident. EKFs using higher order terms exist, however, due to their additional complexity they are not used as frequently. The unscented Kalman filter (UKF) discussed in the next section addressees some of the approximation issues of the EKF.

Another issue that comes up when using the EKF is the derivation of the Jacobian matrices. In many cases, the derivation can be nontrivial, adding difficulty to the EKF's implementation [31]. The UKF achieves the performance of a second order truncated filter [66] without the use of Jacobian or Hessian matrices.

\subsubsection{Tuning the $\mathrm{R}$ and $\mathrm{Q}$ Parameters}

The measurement noise covariance, $R$, and the process noise covariance, $Q$, must be selected appropriately in order for the Kalman filter and the EKF to produce desired results. For instance, consider the following experiment in which the Kalman filter is used to estimate a scalar constant. To make the experiment interesting there is a 
white noise component added to the observations. The process model is

$$
x_{k}=x_{k-1}+v_{k}
$$

where $v_{k} \sim N\left(0,0.001^{2}\right)$. In a similar fashion, the observation model is

$$
y_{k}=x_{k}+w_{k}
$$

where $w_{k} \sim N\left(0,0.1^{2}\right)$. This is analogous to the real life situation of estimating a voltage when the observations are corrupted by a 0.1 volt RMS white measurement noise [68]. The process noise error, $v_{k}$, is relatively small, so for the simulations we set the process noise variance to $Q=1 e-5$. The measurement noise variance, $R$, however, can not be set negligibly small since the measurement noise is substantial. For the first simulation we set $R=(0.1)^{2}$, that is, we set $R$ such that it is equal to the actual measurement noise variance. Since $R$ is set to its true value the filter's performance with this parameter setup should outperform other parameter configurations in terms of responsiveness and estimation variance. This becomes apparent when the results are compared to those of the filter's simulations when $R=1$ and when $R=0.01$.

The simulation results are illustrated in Figures 3.2-3.4. In each of the figures, the dotted line represents the constant value, $x=0.1234$, that is being estimated. Similarly, the crosses in each figure represent the noisy observations used to estimate the constant. The remaining solid line in the figures is the Kalman filter estimate of the constant. Notice that in the simulation where $R$ is increased by a factor of 10 times the true noise variance, the filter responds slower to the noisy measurements. Alternatively, in the simulation where $\mathrm{R}$ is decreased by a factor of 10 , the filter responds much quicker to the noisy measurements.

In practise, the measurement noise covariance can usually be measured prior to 


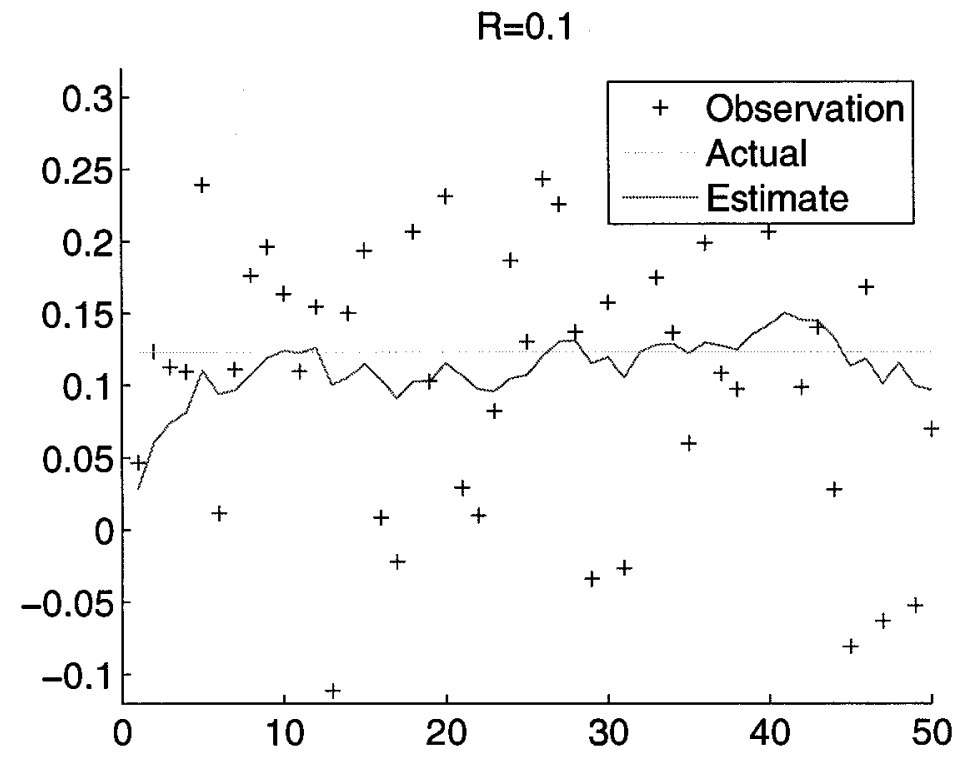

Figure 3.2: First Simulation, $R=0.1$. $\mathrm{R}$ is set to its true value.

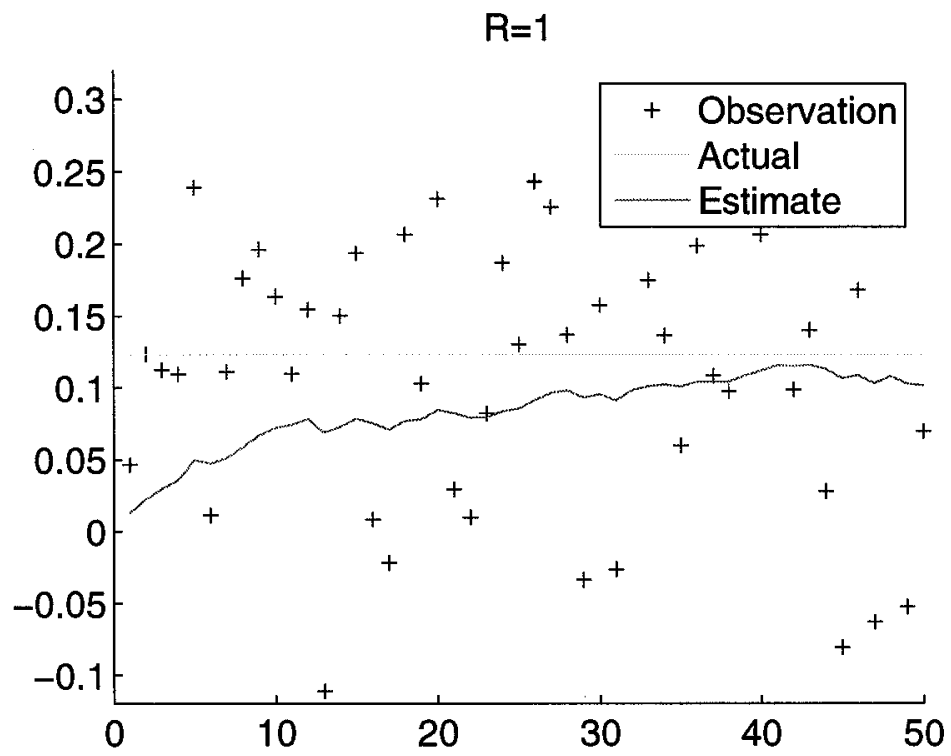

Figure 3.3: Second Simulation, $R=1$. Due to the increase in $\mathrm{R}$ the filter responds slower than in the first case, thus reducing the estimate variance. 
$R=0.01$

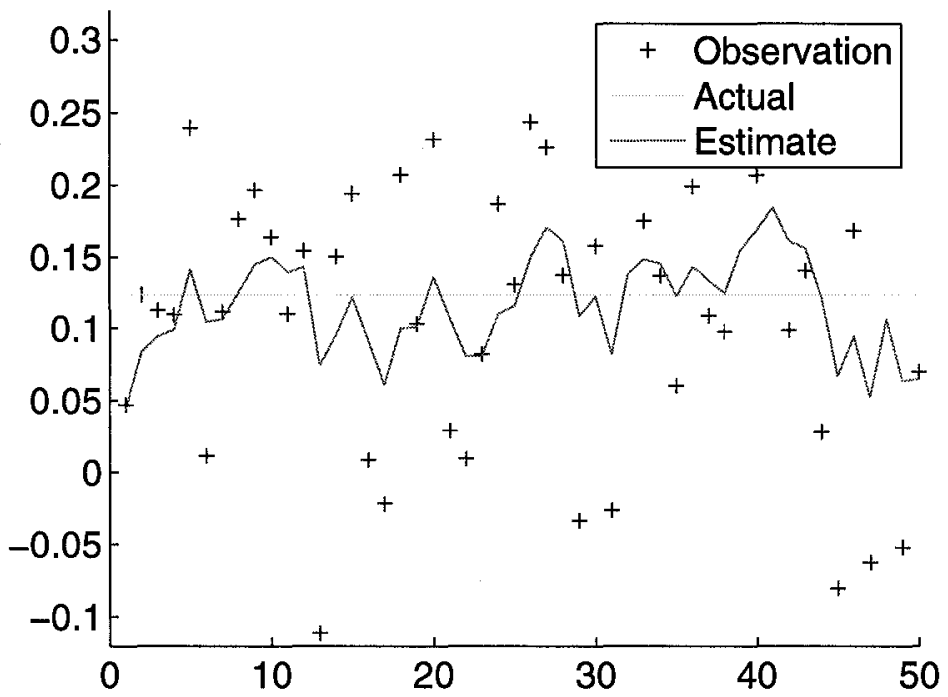

Figure 3.4: Third Simulation, $R=0.01$. Due to the decrease in $\mathrm{R}$ the filter responds quicker than in the first case, thus increasing the estimate variance.

operation of the filter [68]. This measurement can usually be completed off-line with sample data. Commonly, the process being estimated is not directly observable. Consequently, it is generally more difficult to determine the process noise covariance. In cases where the process or measurement noise covariance terms are unknown tuning can be used to achieve preferable results.

\subsection{The Unscented Kalman Filter}

Like the EKF, the UKF is also a sub-optimal recursive MMSE estimator. The main difference between the two filtering techniques is that the UKF does not approximate the nonlinear system and observation models. Alternatively, the UKF method uses the true system and observation models and instead makes an approximation about the distribution of the state variable. The UKF uses what is known as an unscented transform (UT) to estimate the distribution of the state variable. Basically, a set of 
deterministically selected sample points are propagated through the true nonlinear system. These points are used to represent the posterior mean and covariance. The following sections describe the UT, scaled unscented transform (SUT) which is a generalization of the UT, and then finally the UKF, an estimation algorithm that is based on the SUT. The information found in the following sections concerning the UKF is from the sources $[32,66,67,31]$.

\subsubsection{The Unscented Transform}

The unscented transformation is an approximate method for propagating means and covariances through nonlinear transformations. To do this, a set of weighted samples or sigma points $S_{i}=\left\{W_{i}, \chi_{i}\right\}$ are deterministically chosen such that their properties (mean and covariance) represent those of the prior distribution. The set of weighted samples is transformed through the nonlinear system and the transformed set of samples is used to calculate the new mean and covariance statistics. On the surface this seems to be a Monte Carlo method, however, note that no random sampling is implemented, and in fact, only a relatively small number of sample points are required $\left(2 n_{x}+1\right.$ for an $n_{x}$-dimensional state space).

To clarify, let us start by examining the problem at hand. Let $x$ be an $n_{x^{-}}$ dimensional random variable with mean $\bar{x}$ and covariance $P_{x x}$. Let us also introduce another random variable $y$. The random variables are related through the nonlinear transformation

$$
y=f(x)
$$

where $f$ is some nonlinear function. The objective of the unscented transformation is to estimate the mean $\bar{y}$ and covariance $P_{y y}$ of $y$ using the aforementioned sigma points. 
The first issue is how to deterministically select the sigma points such that they represent the proper statistical properties of the prior as discussed above. A scheme to select the points is offered by van der Merwe et al. [66]. It is as follows

$$
\begin{array}{lll}
\chi_{0}=\bar{x} & W_{0}=\frac{\kappa}{n_{x}+\kappa} & i=0 \\
\chi_{i}=\bar{x}+\left(\sqrt{\left(n_{x}+\kappa\right) P_{x}}\right)_{i} & W_{i}=\frac{1}{2\left(n_{x}+\kappa\right)} & i=1, \ldots, n_{x} \\
\chi_{i}=\bar{x}-\left(\sqrt{\left(n_{x}+\kappa\right) P_{x}}\right)_{i} & W_{i}=\frac{1}{2\left(n_{x}+\kappa\right)} & i=n_{x}+1, \ldots, 2 n_{x}
\end{array}
$$

where $\kappa$ is a scaling parameter and $\left(\sqrt{\left(n_{x}+\kappa\right) P_{x}}\right)_{i}$ is the ith column of the matrix square root of $\left(n_{x}+\kappa\right) P_{x}$. The weight of the $i$ th point is denoted $W_{i}$. The sum of the weights is normalized in the sense that $\sum_{i=0}^{2 n_{x}} W_{i}=1$.

The second issue is how to estimate the mean and covariance of $y$. To do this, we start by transforming each of the sigma points with the nonlinear function

$$
\Upsilon_{i}=f\left(\chi_{i}\right) \quad i=0, \ldots, 2 n_{x}
$$

The mean and covariance of $y$ are then calculated as follows

$$
\begin{gathered}
\bar{y}=\sum_{i=0}^{2 n_{x}} W_{i} \Upsilon_{i} \\
P_{y}=\sum_{i=0}^{2 n_{x}} W_{i}\left(\Upsilon_{i}-\bar{y}\right)\left(\Upsilon_{i}-\bar{y}\right)^{T} .
\end{gathered}
$$

These mean and covariance estimates are accurate to the second order of the Taylor series expansion of $f(x)$ for any nonlinear function [66]. Furthermore, the parameter $\kappa$ can be used to scale the errors arising from the higher order terms. This is an improvement over the EKF where the posterior mean and covariance are estimated accurately to the first order term with all higher order terms simply truncated. 
The proper choice of $\kappa$ allows the sigma points to be scaled towards or away from the mean of the prior distribution in order to avoid dimensional effects. Dimensional effects refer to the fact that as the dimension of the state space increases, the radius of the sphere that bounds the sigma points also increases, thus non-local effects get sampled unnecessarily. The problem with choosing $\kappa$, however, is that particular values may cause the calculated covariance to become non-positive semidefinite. The solution to this apparent problem is the scaled unscented transform.

\subsubsection{The Scaled Unscented Transform}

The scaled unscented transform introduces a positive scaling parameter $\alpha: 0 \leq \alpha \leq 1$ in order to minimize higher order dimensional effects. The sigma points are scaled

$$
\chi_{i}^{\prime}=\chi_{0}+\alpha\left(\chi_{i}-\chi_{0}\right) \quad i=0,1, \ldots, 2 n_{x}
$$

before they are transformed. The objective is to insure that the predicted covariance is positive semi-definite and the accuracy to the second order of the mean and covariance is preserved. This is achieved using an auxiliary random variable, $z$. The new formulation of the transform is

$$
z=f^{\prime}(x)=\frac{f[\bar{x}+\alpha(x-\bar{x})]-f(\bar{x})}{\mu}+f(\bar{x})
$$

where $\alpha$ is the positive scaling constant and $\mu$ is a normalization term. The problem is then to estimate the mean $\bar{z}$ and covariance $P_{z z}$ of the auxiliary random variable, $z$. Calculating the expectation of the Taylor series expansion of $\bar{z}$ about $\bar{x}$ and setting $\mu=\alpha^{2}$ we see that it is the same as the Taylor series expansion of $\bar{y}$ up to the second order. The same results hold for the covariances. Please refer to Julier's paper, The 
Scaled Unscented Transform [31], for more details.

The SUT results in the scaled set of sigma points $S^{\prime}=\left(W^{\prime}, \chi^{\prime}\right)$ where

$$
\begin{aligned}
\chi_{i}^{\prime} & =\chi_{0}+\alpha\left(\chi_{i}-\chi_{0}\right) \\
W_{i}^{\prime} & = \begin{cases}W_{0} / \alpha^{2}+\left(1-\frac{1}{\alpha^{2}}\right) & i=0 \\
W_{i} / \alpha^{2} & i \neq 0\end{cases}
\end{aligned}
$$

Furthermore, the sigma point selection and scaling can be combined to save resources [66] by introducing a new variable

$$
\lambda=\alpha^{2}\left(n_{x}+\kappa\right)-n_{x}
$$

and by selecting the sigma point set by:

$$
\begin{array}{ll}
\chi_{0}=\bar{x} & i=0 \\
\chi_{i}=\bar{x}+\left(\sqrt{\left(n_{x}+\lambda\right) P_{x}}\right)_{i} & i=1, \ldots, n_{x} \\
\chi_{i}=\bar{x}-\left(\sqrt{\left.\left(n_{x}+\lambda\right) P_{x}\right)_{i}}\right. & i=n_{x}+1, \ldots, 2 n_{x} \\
W_{0}^{m}=\frac{\lambda}{n_{x}+\lambda} & i=0 \\
W_{i}^{c}=\frac{\lambda}{\left(n_{x}+\lambda\right)}+\left(1-\alpha^{2}+\beta\right) & i=0 \\
W_{i}^{m}=W_{i}^{c}=\frac{1}{2\left(n_{x}+\lambda\right)} & i=1, \ldots, 2 n_{x} .
\end{array}
$$

The $m$ and $c$ superscripts on the weights refer to the mean and covariance terms respectively. The third parameter, $\beta$, is introduced to weight the zeroth sigma point for the covariance calculation. The $\beta$ parameter can be used to minimize higher order errors when the appropriate information about prior distributions of $x$ is known.

The complete SUT can be summarized as follows:

1. Choose the $0 \leq \alpha \leq 1, \beta \geq 0$, and $\kappa \geq 0$ parameters. 
2. Calculate the scaled sigma points $S^{\prime}=\left\{W^{\prime}, \chi^{\prime}\right\}$ using Eqn 3.62 from above.

3. Use the nonlinear transformation to propagate each of the sigma points

$$
\Upsilon_{i}=f\left(\chi_{i}\right) \quad i=0, \ldots, 2 n_{x}
$$

4. Compute the mean and covariances

$$
\begin{gathered}
\bar{y}=\sum_{i=0}^{2 n_{x}} W_{i}^{m} \Upsilon_{i} \\
P_{y}=\sum_{i=0}^{2 n_{x}} W_{i}^{c}\left\{\Upsilon_{i}-\bar{y}\right\}\left\{\Upsilon_{i}-\bar{y}\right\}^{T}
\end{gathered}
$$

\subsubsection{Implementation of the Unscented Kalman Filter}

This section puts forward the UKF algorithm. The UKF is basically an application of the SUT to RMSE estimation. The state variable is redefined as

$$
x_{t}^{a}=\left[x_{t}^{T} v_{t}^{T} n_{t}^{T}\right]^{T}
$$

where the superscript $a$ denotes that this is an augmented random variable. The complete UKF algorithm to estimate $\bar{x}$ and $P$ (as is it presented in [66]) is then:

1. Initialization:

$$
\begin{gathered}
\hat{x}_{0}=E\left[x_{0}\right] \\
P_{0}=E\left[\left(x_{0}-\hat{x}_{0}\right)\left(x_{0}-\hat{x}_{0}\right)^{T}\right] \\
\hat{x}_{0}^{a}=E\left[x^{a}\right]=\left[\hat{x}_{0}^{T} 00\right]^{T}
\end{gathered}
$$




$$
P_{0}^{a}=E\left[\left(x_{0}^{a}-\hat{x}_{0}^{a}\right)\left(x_{0}^{a}-\hat{x}_{0}^{a}\right)^{T}\right]=\left[\begin{array}{ccc}
P_{0} & 0 & 0 \\
0 & \nu & 0 \\
0 & 0 & \eta
\end{array}\right]
$$

2. For $t \in\{1, \ldots, \infty\}$,

(a) Calculate the sigma points as in Eqn 3.62:

$$
\chi_{t-1}^{a}=\left[\hat{x}_{t-1}^{a}, \hat{x}_{t-1}^{a} \pm \sqrt{\left(n_{a}+\lambda\right) P_{t-1}^{a}}\right]
$$

(b) Propagate sigma points through the transition function:

$$
\chi_{t / t-1}^{x}=f\left(\chi_{t-1}^{x}, \chi_{t-1}^{v}\right)
$$

(c) Preditct state and covariance using the weighted sigma points:

$$
\begin{gathered}
\hat{x}_{t / t-1}=\sum_{i=1}^{2 n_{a}} W_{i}^{(m)} \chi_{i, t / t-1}^{x} \\
P_{t / t-1}=\sum_{i=1}^{2 n_{a}} W_{i}^{(c)}\left[\chi_{i, t / t-1}^{x}-\hat{x}_{t / t-1}\right]\left[\chi_{i, t / t-1}^{x}-\hat{x}_{t / t-1}\right]^{T}
\end{gathered}
$$

(d) Project sigma points through the observation function:

$$
\Upsilon_{t / t-1}=H\left(\chi_{t / t-1}^{x}, \chi_{t / t-1}^{n}\right)
$$

(e) Compute the predicted measurement and predicted measurement covariance:

$$
\hat{y}_{t / t-1}=\sum_{i=0}^{2 n_{a}} W_{i}^{(m)} \Upsilon_{i, t / t-1}
$$




$$
P_{\hat{y}_{t} \hat{y}_{t}}=\sum_{i=1}^{2 n_{a}} W_{i}^{(c)}\left[\Upsilon_{i, t / t-1}^{x}-\hat{y}_{t / t-1}\right]\left[\Upsilon_{i, t / t-1}-\bar{y}_{t / t-1}\right]^{T}
$$

(f) Calculate the state-measurement matrix:

$$
P_{\hat{x}_{t} \hat{y}_{t}}=\sum_{i=1}^{2 n_{a}} W_{i}^{(c)}\left[\chi_{i, t / t-1}-\hat{x}_{t / t-1}\right]\left[\Upsilon_{i, t / t-1}-\hat{x}_{t / t-1}\right]^{T}
$$

(g) Use the state-measurement matrix to calculate the UKF gain:

$$
K_{t}=P_{\hat{x}_{t} \hat{y}_{t}} P_{\hat{y}_{t} \hat{y}_{t}}^{-1}
$$

(h) Update the state and covariance:

$$
\begin{gathered}
\hat{x}_{t}=\hat{x}_{t / t-1}+K_{t}\left(y_{t}-\hat{y}_{t / t-1}\right) \\
P_{t}=P_{t / t-1}-K_{t} P_{\hat{y}_{t} \hat{y}_{t}} K_{T}^{t}
\end{gathered}
$$

where $x^{a}=\left[x^{T} v^{T} n^{T}\right]^{T}, \chi^{a}=\left[\left(\chi^{x}\right)^{T}\left(\chi^{v}\right)^{T}\left(\chi^{n}\right)^{T}\right]^{T}$, and $n_{a}=n_{x}+n_{v}+n_{n}$. The main advantage of the UKF over the EKF is that no Jacobians or Hessians are calculated at any point in the algorithm. The computational cost of the UKF is of the same order as the computational cost of the EKF. Superior performance of the UKF over the EKF in areas of accuracy and robustness have been reported recently in various publications including $[67,66,32,44,10]$. 


\subsection{Sequential Monte Carlo Methods (Particle Fil- ters)}

From Chapter 1, the goal of SMC methods is to recursively in time, $t$, estimate the posterior distribution $p\left(x_{0: t} \mid y_{1: t}\right)$ and ultimately the expectations

$$
E_{p\left(x_{0: t} \mid y_{1: t}\right)}\left[\psi_{t}\left(x_{0: t}\right)\right] \triangleq \int \psi_{t}\left(x_{0: t}\right) p\left(x_{0: t} \mid y_{1: t}\right) d x_{0: t}
$$

for some function $\psi_{t}$.

To estimate the posterior distribution we can use a two stage recursive Bayesian estimation method. The first stage is known as the update stage. In this stage, the prior distribution over the system state at the current time is known and an observation is made. The posterior is then updated to reflect the newly available information.

$$
\begin{aligned}
p\left(x_{t} \mid y_{1: t}\right) & =\frac{p\left(x_{t}, y_{1: t}\right)}{p\left(y_{1: t}\right)} \\
& =\frac{p\left(y_{t} \mid x_{t}, y_{1: t-1}\right) p\left(x_{t}, y_{1: t-1}\right)}{p\left(y_{t} \mid y_{1: t-1}\right) p\left(y_{1: t-1}\right)} \\
& =\frac{p\left(y_{t} \mid x_{t}\right) p\left(x_{t} \mid y_{1: t-1}\right)}{p\left(y_{t} \mid y_{1: t-1}\right)} \\
& =\frac{p\left(y_{t} \mid x_{t}\right) p\left(x_{t} \mid y_{1: t-1}\right)}{p\left(y_{t} \mid y_{1: t-1}\right)}
\end{aligned}
$$

where

$$
\begin{aligned}
p\left(y_{t} \mid y_{1: t-1}\right) & =\int p\left(y_{t}, x_{t} \mid y_{1: t-1}\right) d x_{t} \\
& =\int p\left(y_{t} \mid x_{t}\right) p\left(x_{t} \mid y_{1: t}\right) d x_{t}
\end{aligned}
$$

The second stage is the prediction stage. Using the posterior distribution and the 
system model, the next prior distribution is predicted.

$$
\begin{aligned}
p\left(x_{t} \mid y_{1: t-1}\right) & =\int p\left(x_{t}, x_{t-1} \mid y_{1: t-1}\right) d x_{t-1} \\
& =\int p\left(x_{t} \mid x_{t-1}\right) p\left(x_{t-1} \mid y_{1: t-1}\right) d x_{t-1}
\end{aligned}
$$

\subsubsection{Perfect Monte Carlo Simulation}

Monte Carlo simulation uses a set of weighted particles (samples), drawn from the posterior distribution of the model, in order to approximate integrals as discrete sums. Given the set of $N_{p}$ random samples, $\left\{x_{0: t}^{(i)}: i=1, \ldots, n\right\}$, the posterior distribution can be approximated as

$$
\hat{p}\left(x_{0: t} \mid y_{1: t}\right)=\frac{1}{N_{p}} \sum_{i=1}^{N_{p}} w_{0: t}^{(i)} \delta\left(x_{0: t}-x_{0: t}^{(i)}\right)
$$

where $\delta(\cdot)$ is the Dirac delta function ${ }^{1}$.

The expectation of some function $\psi\left(x_{0: t}\right)$ can be approximated by the following Monte Carlo estimate

$$
\bar{E}\left[\psi\left(x_{0: t}\right)\right]=\frac{1}{N_{p}} \sum_{i=1}^{N_{p}} \psi\left(x_{0: t}^{(i)}\right)
$$

The samples $x_{0: t}^{(i)}$ are referred to as particles. The particles must be independent and identically distributed for the approximation to be valid. Referring to Appendix A, we know from the strong law of large numbers

$$
\bar{E}\left[\psi\left(x_{0: t}\right)\right] \underset{N_{p} \rightarrow \infty}{\stackrel{a . s .}{\longrightarrow}} E\left[\psi\left(x_{0: t}\right)\right] .
$$

\footnotetext{
${ }^{1}$ First introduced by physicist Paul Dirac, the Dirac delta function, $\delta(x)$ has the value of infinity when $x=0$ and the value zero elsewhere. The integral of the function from negative infinity to positive infinity is one.
} 
Furthermore, if the posterior variance exists and is bounded, we know from the central limit theorem that $\bar{E}\left[\psi\left(x_{0: t}\right)\right]$ tends to a normal distribution as $n \longrightarrow \infty$.

\subsubsection{Bayesian Importance Sampling}

It is usually not possible to sample from the posterior distribution directly. By drawing samples from a proposal distribution, $q\left(x_{0: t} \mid y_{1: t}\right)$, this problem can be circumvented. First, define the unnormalized importance weights

$$
w_{t} \equiv \frac{p\left(y_{1: t} \mid x_{0: t}\right) p\left(x_{0: t}\right)}{q\left(x_{0: t} \mid y_{1: t}\right)} .
$$

Next, note that

$$
\begin{aligned}
p\left(y_{1: t}\right) & =\int p\left(y_{1: t}, x_{0: t}\right) d x_{0: t} \\
& =\int \frac{p\left(y_{1: t} \mid x_{0: t}\right) p\left(x_{0: t}\right) q\left(x_{0: t} \mid y_{1: t}\right)}{q\left(x_{0: t} \mid y_{1: t}\right)} d x_{0: t} \\
& =\int w_{t}\left(x_{0: t}\right) q\left(x_{0: t} \mid y_{1: t}\right) d x_{0: t} .
\end{aligned}
$$

Now the expectation of some function $\psi\left(x_{0: t}\right)$ can be computed as follows:

$$
\begin{aligned}
E\left[\psi\left(x_{0: t}\right)\right] & =\int \psi\left(x_{0: t}\right) p\left(x_{0: t} \mid y_{1: t}\right) d x_{0: t} \\
& =\int \frac{\psi\left(x_{0: t}\right) p\left(x_{0: t} \mid y_{1: t}\right) q\left(x_{0: t} \mid y_{1: t}\right)}{q\left(x_{0: t} \mid y_{1: t}\right)} d x_{0: t} \\
& =\int \frac{\psi\left(x_{0: t}\right) p\left(y_{1: t} \mid x_{0: t}\right) p\left(x_{0: t}\right) q\left(x_{0: t} \mid y_{1: t}\right)}{p\left(y_{1: t}\right) q\left(x_{0: t} \mid y_{1: t}\right)} d x_{0: t} \\
& =\int \frac{\psi\left(x_{0: t}\right) w_{t}\left(x_{0: t}\right) q\left(x_{0: t} \mid y_{1: t}\right)}{p\left(y_{1: t}\right)} d x_{0: t} \\
& =\frac{1}{p\left(y_{1: t}\right)} \int \psi\left(x_{0: t}\right) w_{t}\left(x_{0: t}\right) q\left(x_{0: t} \mid y_{1: t}\right) d x_{0: t} \\
& =\frac{\int \psi\left(x_{0: t}\right) w_{t}\left(x_{0: t}\right) q\left(x_{0: t} \mid y_{1: t}\right) d x_{0: t}}{\int w_{t}\left(x_{0: t}\right) q\left(x_{0: t} \mid y_{1: t}\right) d x_{0: t}}
\end{aligned}
$$


By drawing independent random samples from $q\left(x_{0: t} \mid y_{1: t}\right)$ and using Monte Carlo integration, an approximate expectation can be obtained:

$$
\begin{aligned}
\bar{E}\left[\psi\left(x_{0: t}\right)\right] & =\frac{\frac{1}{N_{p}} \sum_{i=1}^{N_{p}} \psi\left(x_{0: t}^{i}\right) w_{t}\left(x_{0: t}^{i}\right)}{\frac{1}{N_{p}} \sum_{i=1}^{N_{p}} w_{t}\left(x_{0: t}^{i}\right)} \\
& =\sum_{i=1}^{N_{p}} \psi\left(x_{0: t}^{i}\right) \tilde{w}_{t}\left(x_{0: t}^{i}\right) .
\end{aligned}
$$

where $\tilde{w}_{t}^{(i)}$ represents the normalized importance weights. The point mass estimate of the posterior distribution is

$$
\hat{p}\left(x_{0: t} \mid y_{1: t}\right)=\frac{1}{N_{p}} \sum_{i=1}^{N_{p}} w_{0: t}^{(i)} \delta\left(x_{0: t}-x_{0: t}^{(i)}\right)
$$

as the set of points and respective weights $\left\{x_{0: t}^{i}, w_{t}^{i}\right\}_{i=1}^{N_{p}}$ is a random measure characterizing the posterior pdf $p\left(x_{0: t} \mid y_{1: t}\right)$.

\subsubsection{Sequential Importance Sampling}

In order to make Bayesian importance sampling more practical it is convenient to be able to calculate the particle weights recursively. We start by recalling that the states are assumed to correspond to a Markov process. Additionally, the observations are assumed to be conditionally independent given the states. From these assumptions:

$$
p\left(x_{0: t}\right)=p\left(x_{0}\right) \sum_{j=1}^{t} p\left(x_{j} \mid x_{j-1}\right)
$$


and

$$
p\left(y_{1: t} \mid x_{0: t}\right)=\sum_{j=1}^{t} p\left(y_{j} \mid x_{j}\right)
$$

We also note that the proposal distribution can be chosen [3] to factorize as follows:

$$
\begin{aligned}
q\left(x_{0: t} \mid y_{1: t}\right) & =q\left(x_{0}\right) \sum_{j=1}^{t} q\left(x_{j} \mid x_{0: j-1}, y_{1: j}\right) \\
& =q\left(x_{0: t-1} \mid y_{1: t-1}\right) q\left(x_{t} \mid x_{0: t-1}, y_{1: t}\right)
\end{aligned}
$$

By substituting these results into Eqn 3.89 a sequential method for calculating the weights is achieved

$$
\begin{aligned}
w_{t} & =\frac{p\left(y_{1: t} \mid x_{0: t}\right) p\left(x_{0: t}\right)}{q\left(x_{0: t} \mid y_{1: t}\right)} \\
& =\frac{p\left(y_{1: t} \mid x_{0: t}\right) p\left(x_{0: t}\right)}{q\left(x_{0: t-1} \mid y_{1: t-1}\right) q\left(x_{t} \mid x_{0: t-1}, y_{1: t}\right)} \\
& =w_{t-1} \frac{p\left(y_{1: t} \mid x_{0: t}\right) p\left(x_{0: t}\right)}{p\left(y_{1: t-1} \mid x_{0: t-1}\right) p\left(x_{0: t-1}\right) q\left(x_{t} \mid x_{0: t-1}, y_{1: t}\right)} \\
& =w_{t-1} \frac{p\left(x_{t} \mid x_{t-1}\right) p\left(y_{t} \mid x_{t}\right)}{q\left(x_{t} \mid x_{0: t-1}, y_{1: t}\right)} .
\end{aligned}
$$

Eqn 3.97 requires that the path $x_{0: t-1}^{i}$ and history of observations $y_{1: t-1}$ be stored. Commonly, only the filtered estimate $p\left(x_{t} \mid y_{t}\right)$ is required. In this case, we can set $q\left(x_{t} \mid x_{0: t-1}, y_{1: t}\right)=q\left(x_{t} \mid x_{t-1}, y_{t}\right)$ such that the weights are only dependent upon $x_{t-1}$ and $y_{t}$. The estimate is then

$$
\hat{p}\left(x_{t} \mid y_{t}\right)=\sum_{i=1}^{N_{p}} \tilde{w}_{t}^{(i)} \delta\left(x_{t}-x_{t}^{(i)}\right)
$$

To summarize, the sequential importance sampling (SIS) algorithm starts by sampling the proposal function. The relevant likelihood and transition probabilities are evaluated using the samples. The importance weights are then recursively computed 
input: $\left\{x_{t-1}^{i}, w_{t-1}^{i}\right\}_{i=1}^{N_{p}}, y_{t}$

For $i=1: N_{p}$

-Draw $x_{t}^{i} \sim q\left(x_{t} \mid x_{t-1}^{i}, y_{t}\right)$

-Assign each particle a respective weight $w_{t}^{i}$ using Eqn 3.97

End

output: $\left\{x_{t}^{i}, w_{t}^{i}\right\}_{i=1}^{N_{p}}$

using Eqn 3.97. The SIS method acts as a mechanism to generate the estimates of Eqn 3.98. Pseudo-code for the algorithm is given in Table 3.1.

Choosing a proper proposal function is a critical design issue when using importance sampling algorithms. The optimal function in terms of minimizing the variance of the importance weights has been shown to be [3]

$$
q\left(x_{t} \mid x_{t-1}^{i}, y_{t}\right)_{\text {opt }}=p\left(x_{t} \mid x_{t-1}^{i}, y_{t}\right)
$$

The problem with selecting this particular proposal density is that it may not be possible to sample from it [3]. An example of where this choice has been used is Bayesian missing-data problems $[42,48]$. Another example is jump Markov linear systems for tracking maneuvering targets [16].

The most popular choice of distribution for the proposal is the prior distribution, i.e.,

$$
q\left(x_{t} \mid x_{t-1}^{i}, y_{t}\right)=p\left(x_{t} \mid x_{t-1}\right)
$$

Using the prior as the proposal density the importance weight calculation simplifies 
to

$$
\begin{aligned}
w_{t} & =w_{t-1} \frac{p\left(x_{t} \mid x_{t-1}\right) p\left(y_{t} \mid x_{t}\right)}{q\left(x_{t} \mid x_{0: t-1}, y_{1: t}\right)} \\
& =w_{t-1} \frac{p\left(x_{t} \mid x_{t-1}\right) p\left(y_{t} \mid x_{t}\right)}{p\left(x_{t} \mid x_{t-1}\right)} \\
& =w_{t-1} p\left(y_{t} \mid x_{t}\right)
\end{aligned}
$$

Despite having higher Monte Carlo variation than the optimal proposal, the prior is very popular due to its convenience. The prior distribution has been used in many applications [7, 26, 38, 48].

\subsubsection{Resampling and the Generic Particle Filter}

Degeneracy is a problem that commonly hinders the SIS particle filter's performance. Degeneracy, in the realm of particle filters, is when after a few iterations of the algorithm, all but one of the particles have a negligible weight. It is impossible to avoid degeneracy as the variance of the importance weights can only increase stochastically with time [3]. To counteract degeneracy, particles with relatively little contribution to the approximation of $p\left(x_{t} \mid y_{1: t}\right)$ must be updated. This process is known as resampling. Liu and Chen [48] introduced two methods of detecting when to resample. The first is a deterministic schedule that conducts resampling at predefined times $t_{0}, 2 t_{0}, \ldots$ where $t_{0}$ depends on the problem. In this case, setting the value of $t_{0}$ would require experimentation. The second method they suggest is a dynamic schedule. The algorithm would have to monitor the level of degeneracy and then respond by resampling when certain thresholds are crossed.

The effective sample size, $n_{\text {eff }}$, is used as a measure of degeneracy of particle 
filtering algorithms [3]. It is defined as

$$
n_{e f f}=\frac{N_{p}}{1+\operatorname{var}\left(\frac{p\left(x_{t}^{i} \mid y_{1: t}\right)}{q\left(x_{t}^{i} \mid x_{t-1}^{i}, y_{t}\right)}\right)}
$$

Usually this expression cannot be evaluated, thus, an approximation is used, specifically,

$$
\hat{n}_{e f f}=\frac{1}{\sum_{i=1}^{N_{p}}\left(w_{t}^{i}\right)^{2}}
$$

where $w_{t}^{i}$ are the normalized weights. Hence, small $\hat{n}_{e f f}$ is an indication of harsh degeneracy.

Resampling is used to focus on the heavily weighted particles and eliminate particles with small weights. A new set of particles $\left\{x_{t}^{i *}\right\}^{N_{p_{i=1}}}$ is generated by sampling with replacement from the approximation

$$
\hat{p}\left(x_{0: t} \mid y_{1: t}\right)=\frac{1}{N_{p}} \sum_{i=1}^{N_{p}} w_{0: t}^{(i)} \delta\left(x_{0: t}-x_{0: t}^{(i)}\right)
$$

$N_{p}$ times. In this scenario, the weights act as the probabilities such that $\mathcal{P}\left(x_{t}^{i *}=\right.$ $\left.x_{t}^{j}\right)=w_{t}^{j}$. After this selection is complete, all the weights of the new particles are set so that $w_{t}^{i *}=1 \mid N_{p}$. An algorithm for resampling proposed by Kitawaga [38] is depicted in Table 3.2. A graphical interpretation of the process is illustrated in Figure 3.5. For an introduction and comparison of a variety of resampling schemes for particle filters see [14].

Using the resampling method in conjunction with the SIS particle filter we can compose what is known as the generic particle filter. It can be used as the basic skeleton of many particle filtering algorithms. The flow of the algorithm is illustrated in Figure 3.6. 
Table 3.2: Particle Filter Resampling Algorithm [3]

input: $\left\{x_{t-1}^{i}, w_{t-1}^{i}\right\}_{i=1}^{N_{p}}$

Initialize the cdf: $c_{1}=0$

For $i=2: N_{p}$

End

-Construct the cdf: $c_{1}=c_{i-1}+w_{t}^{i}$

Set: $i=1$

Draw a starting point: $u_{1} \sim \mathcal{U}\left[0, N_{p}^{-1}\right]$

For $j=1: N_{p}$

-Move through the cdf: $u_{j}=u_{1}+N_{p}^{-1}(j-1)$

-While $u_{j}>c_{i}$

$$
i=i+1
$$

End

-Set sample: $x_{t}^{j *}=x_{t}^{i}$

-Set weight: $w_{t}^{j}=1 \mid N_{p}$

End

output: $\left\{x_{t}^{j *}, w_{t}^{j}\right\}_{j=1}^{N_{p}}$

Table 3.3: Generic particle filter framework.

Input: $\left[\left\{x_{k-1}^{(i)}, w_{k-1}^{(i)}\right\}_{i=1}^{N_{p}}, y_{k}\right]$

For $i=1: N_{p}$

-Draw samples $x_{k}^{(i)} \sim q\left(x_{k} \mid x_{0: k-1}^{(i)}, y_{1: k}\right)$

-Update importance weights of particles $w_{k}^{(i)}=w_{k-1}^{(i)} \frac{p\left(y_{k} \mid x_{k}^{(i)}\right) p\left(x_{k}^{(i)} \mid x_{k-1}^{(i)}\right)}{q\left(x_{k} \mid x_{0: k-1}^{(i)}, y_{1: k}\right)}$

End

For $i=1: N_{p}$

-Normalize weights $w_{k}^{(i)}=w_{k}^{(i)}\left[\sum_{j=1}^{N_{p}} w_{k}^{(j)}\right]^{-1}$

End

Resample

Output: $\left[\left\{x_{k}^{(i)}, w_{k}^{(i)}\right\}_{i=1}^{N_{p}}\right]$ 


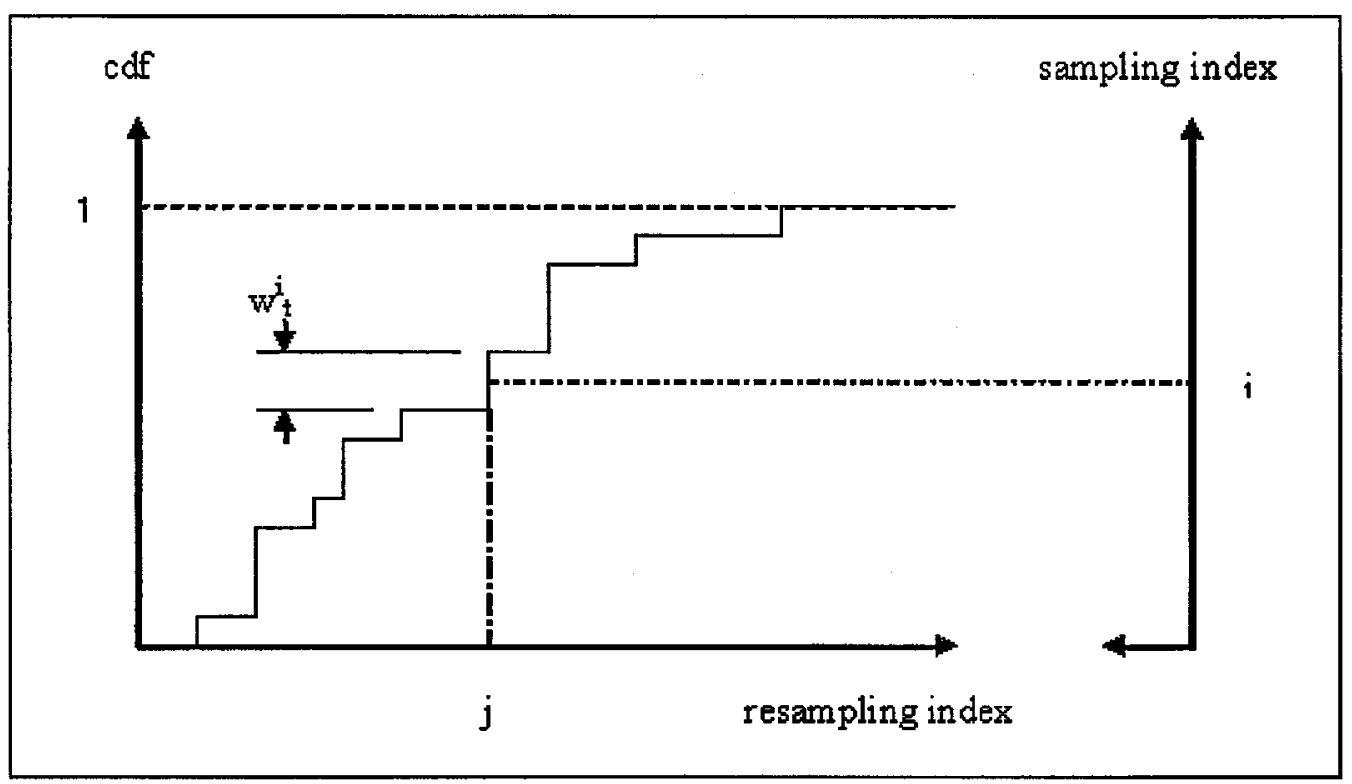

Figure 3.5: Graphical Interpretation of Resampling: the random measure $\left\{x_{t}^{i}, w_{t}^{i}\right\}_{i=1}^{N_{p}}$ is mapped into an equally weighted random measure $\left\{x_{t}^{j *}, w_{t}^{j}\right\}_{j=1}^{N_{p}}$. Figure motivated by [13].

\subsubsection{Sample Importance Resampling}

A SMC method of particular interest is the sample importance resampling (SIR) filter. It was first proposed in 1993 by Gordon et al. [26]. The SIR filter is important because its simplicity makes it easy to implement and very practical for many applications. It will be one of the SMC methods used for comparative purposes later in this thesis.

The SIR algorithm can be derived from the generic particle filter. First is the choice of proposal density. For the SIR filter we use the prior distribution as the proposal, $q\left(x_{t} \mid x_{t-1}^{i}, y_{t}\right)=p\left(x_{t} \mid x_{t-1}\right)$. Thus, the weight calculation simplifies as shown in Eqn 3.101. The second choice that simplifies the SIR particle filter is that of resampling at every time step. This does two very nice things. First, there is no need to figure out a threshold value for resampling. This saves both calculations at run time and preparation time when setting up the filter. Secondly, since all the weights 


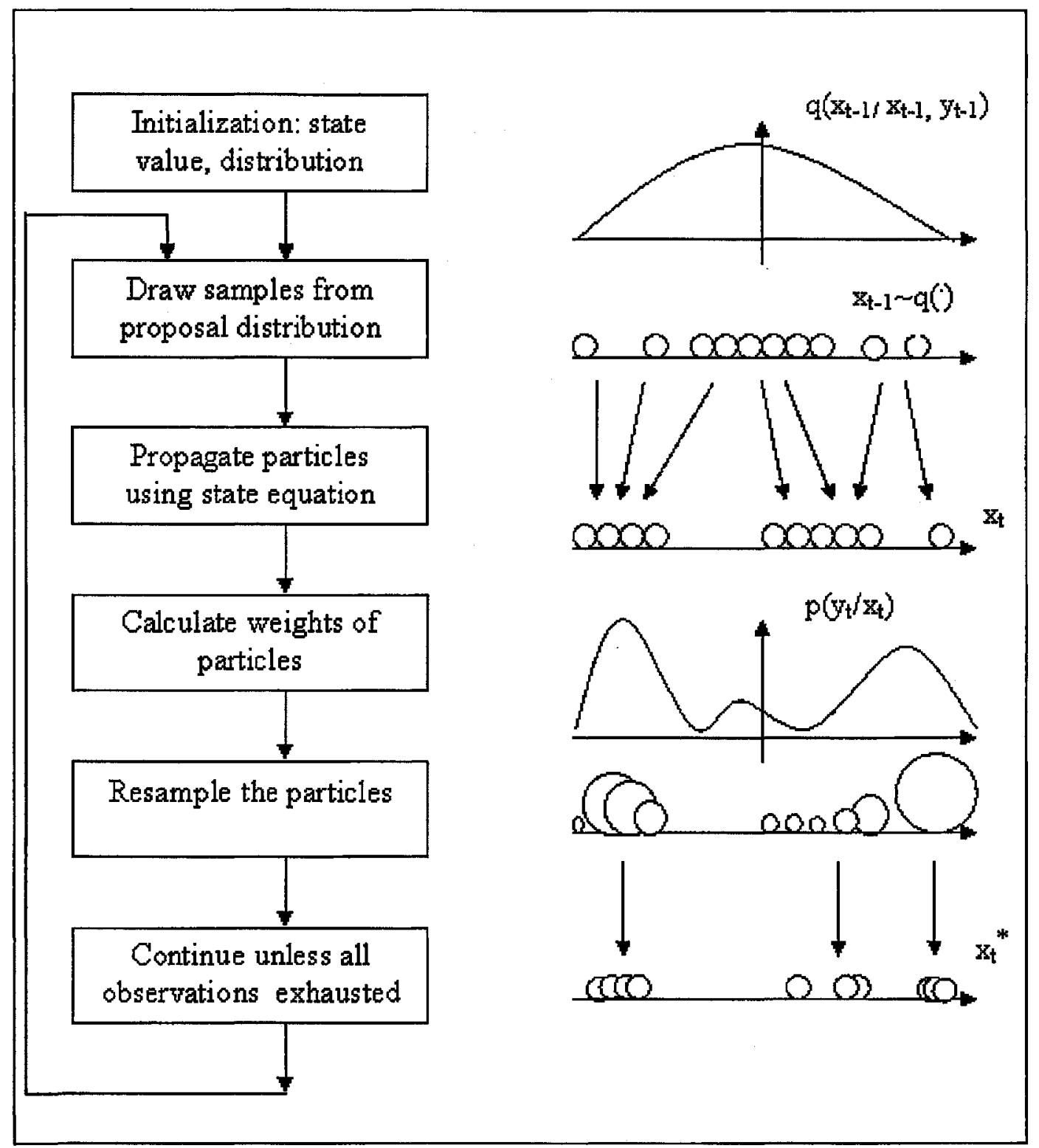

Figure 3.6: Generic Particle Filter Flow Diagram: particles are sampled, propagated, weighted, and then resampled before the process is repeated with a new observation. The particles are represented by circles. The radius of the circle corresponds to the particle's weight. 
Table 3.4: SIR Particle Filter Algorithm [3]

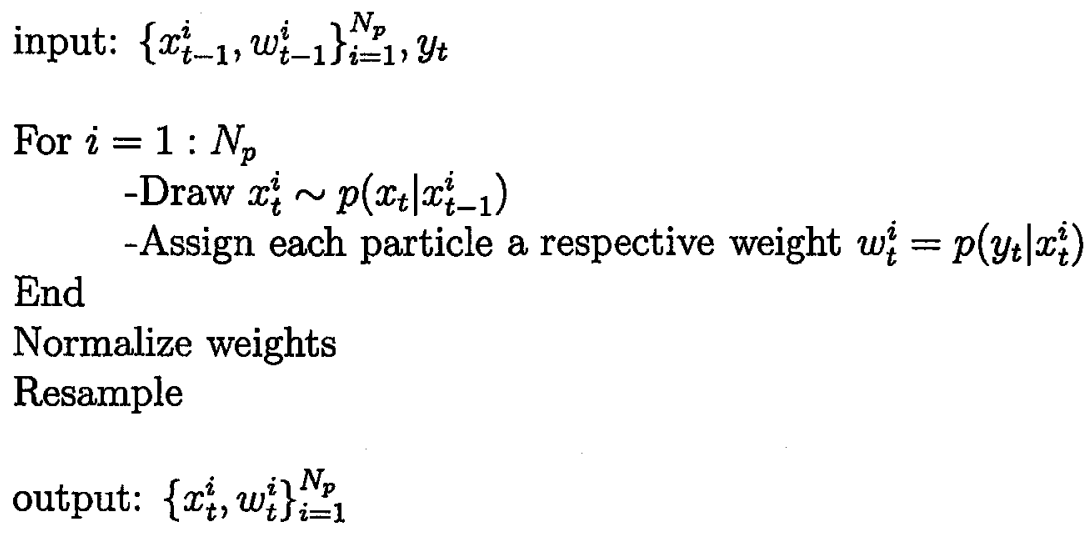

are set to the same value at every time step, the weight calculation can be simplified further, i.e.,

$$
\begin{aligned}
w_{t} & =w_{t-1} p\left(y_{t} \mid x_{t}\right) \\
& =p\left(y_{t} \mid x_{t}\right)
\end{aligned}
$$

There are disadvantages to using the SIR particle filter. Since the proposal density is chosen to be independent of the current observations, the state space is explored without all the available knowledge. As a result, the filter may be sensitive to outliers. Additionally, the choice to resample at every time step may lead to a loss of diversity among particles.

Pseudo-code for the SIR particle filtering algorithm is given in Table 3.4.

\subsubsection{Extended and Unscented Kalman Particle Filters}

Similarly to the SIR particle filter, the extended Kalman particle filter (EKPF) and the unscented Kalman Particle filter (UKPF) are variations on the GPF framework. 
They use the EKF and the UKF respectively, in order to propagate the particles to the current state. This is achieved by using the EKF and the UKF as the proposal densities. This idea was first proposed in 2000 by van der Merwe et al. [66]. Although these methods do not simplify the filter algorithm as the prior proposal does, they do offer improved accuracy in estimation over the SIR method. This is due mostly to the fact that they incorporate the newest data into the particle propagation and state exploration step. These filters will be used as a basis for comparison in the next chapter.

\subsubsection{The Auxiliary Particle Filter}

The Auxiliary Particle Filter (APF) was introduced by Pitt and Shephard and is more robust to outliers than the GPF [58]. The method introduces the joint importance density $q\left(x_{k}, i \mid y_{1: k}\right)$ where $i$, the index of the particle, is an auxiliary variable. Similarly, a characterization of the state transition, $u_{t}$ is also introduced to aid in the simulation. Pitt and Shephard suggest using either the mean, the mode, or a sample from $p\left(x_{k} \mid x_{k-1}\right)$ for $u_{k}$. The framework for the auxiliary particle filter is found in Table 3.5.

Arulampalam et al. [3] point out that when the process noise is small, the point estimates $u_{k}^{(i)}$ may characterize the density $p\left(x_{k} \mid x_{k-1}\right)$ well. In these cases, the APF will often be less sensitive to outliers than the GPF. However, in the alternate scenario, where the process noise is large, the point estimates $u_{k}^{(i)}$ may characterize the density $p\left(x_{k} \mid x_{k-1}\right)$ poorly. In this case, the APF may degrade the filter's performance.

As is the case with the GPF, selection of a proposal density is a key design issue when building an APF. The choice of Pitt and Shephard [58] was the prior distribution. Again, it offers nice simplifications in the APF mathematics making it easy to work with. This thesis proposes the use of the EKF and the UKF as 
Table 3.5: Auxiliary particle filter framework.

Input: $\left[\left\{x_{k-1}^{(i)}, w_{k-1}^{(i)}\right\}_{i=1}^{N_{p}}, y_{k}\right]$

For $i=1: N_{p}$

-Calculate some characterization of $x_{k}$ given $x_{k-1}$ denoted $u_{k}^{(i)}$

-Update importance weights of particles $w_{k}^{(i)}=w_{k-1}^{(i)} \frac{p\left(y_{k} \mid u_{k}^{(i)}\right) p\left(x_{k}^{(i)} \mid x_{k-1}^{(i)}\right)}{q\left(x_{k} \mid i, y_{1: k}\right)}$

End

For $i=1: N_{p}$

-Normalize weights $w_{k}^{(i)}=w_{k}^{(i)}\left[\sum_{j=1}^{N_{p}} w_{k}^{(j)}\right]^{-1}$

End

Resample:

Initialize the cdf: $c_{1}=0$

For $i=2: N_{p}$

-Construct the cdf: $c_{1}=c_{i-1}+w_{t}^{i}$

End

Set: $i=1$

Draw a starting point: $u_{1} \sim \mathcal{U}\left[0, N_{p}^{-1}\right]$

For $j=1: N_{p}$

-Move through the cdf: $u_{j}=u_{1}+N_{p}^{-1}(j-1)$

-While $u_{j}>c_{i}$

$$
i=i+1
$$

-End

-Draw samples $x_{k}^{(j)} \sim q\left(x_{k} \mid i, y_{k}\right)$

-Update importance weights of particles $w_{k}^{(j)}=\frac{p\left(y_{k} \mid x_{k}^{(j)}\right) p\left(x_{k}^{(j)} \mid x_{k-1}^{(i)}\right)}{p\left(y_{k} \mid u_{k}^{(i)}\right) q\left(x_{k}^{(j)} \mid i, y_{k}\right)}$

End

For $i=1: N_{p}$

-Normalize weights $w_{k}^{(i)}=w_{k}^{(i)}\left[\sum_{j=1}^{N_{p}} w_{k}^{(j)}\right]^{-1}$

End

Output: $\left[\left\{x_{k}^{(i)}, w_{k}^{(i)}\right\}_{i=1}^{N_{p}}\right]$ 
the proposal density in the APF framework. These filters will be referred to as the Auxiliary Extended Kalman Particle Filter (AEKPF) and the Auxiliary Unscented Kalman Particle Filter (AUKPF), respectively. In many cases, the EKF and the UKF should offer better results as they do in the GPF framework.

\subsection{Chapter Summary}

This chapter starts by introducing Gauss's work on linear regression and then moves forward through time describing relevant ideas in filtering theory and state estimation up to the state of the art. Although Kalman's method is still widely used, the newer sequential Monte Carlo method is gaining popularity. Similar to the Kalman filter that took advantage of the computational power available during its day, the particle filter is too making the most of the currently available machines. The end of this chapter discusses particle filtering in detail including the generic and the auxiliary particle filter frameworks, choice of proposal, and degeneracy. The novel ideas of this thesis, namely the AEKPF and AUKPF algorithms are discussed to conclude the chapter. 


\section{Chapter 4}

\section{Synthetic Nonlinear Estimation Problems}

\subsection{Metrics for Comparison and Complexity}

The fourth and fifth chapters of this thesis contain comparisons and analysis of the filters presented in Chapter 3. The main metric used for the comparisons and analysis is a RMSE calculation of the estimated state with respect to the actual state. Each experiment is composed of a number of trial runs and each trial run contains a specific number of observations. For every filter a RMSE value is calculated for each trial run. For a specific filter, the RMSE value of a trial run is calculated using the errors over all the observations of that particular trial run. The RMSE value reported in the comparison tables is then the mean RMSE value over all the trial runs for the experiment of interest. The variance value that is reported is the variance calculated from all the RMSE values of a specific filter for the experiment.

Each trial run of an experiment uses a different random seed, hence, produces different results for the actual target trajectory and for the filters' performances. Ap- 
pendix $\mathrm{B}$ contains a listing of the various types of random numbers that are generated along with the algorithms used to generate them.

Some of the tables list results for the filters using various numbers of particles. The results of the EKF and the UKF do not change with respect to the number of particles because the EKF and UKF are not particle based algorithms.

All of the particle filter algorithms that are presented have the same computational complexity. The cost of the algorithms varies linearly with the number of particles used for the calculations. That being said, the algorithms with more advanced particle transitions and weight calculations such as the AEKPF require more calculations at run time compared to an algorithm that has simpler calculations such as the SIRPF.

\subsection{Gaussian Noise Model Results}

\subsubsection{Model Description}

The synthetic experiment discussed in this section is similar to the one used by van der Merwe et al. [66]. For this experiment, the nonlinear process model is as follows

$$
x_{k}=1+\sin (\omega \pi k)+\phi_{1} x_{k-1}+v_{k}
$$

where $\omega=0.04$ and $\phi_{1}=0.5$ are scalar parameters and $v_{k}$ is a zero mean Gaussian random variable $v_{k} \sim N\left(0, \sigma_{v}^{2}\right)$ with $\sigma_{v}^{2}=1$ modeling the process noise. There is a non-stationary element of the experiment present in the observation model

$$
y_{k}= \begin{cases}\phi_{2} x_{k}-2+w_{k} & (k \leq 20) \\ \phi_{3} x_{k}^{2}+w_{k} & (20<k \leq 40) \\ \phi_{4} x_{k}^{3}+w_{k} & (k>40)\end{cases}
$$


where $\phi_{2}=0.2, \phi_{3}=0.5$, and $\phi_{4}=0.1$ are scalar parameters and $w_{k}$, the observation noise, is modelled as a Gaussian random variable, $w_{k} \sim N\left(0, \sigma_{w}^{2}\right)$, where $\sigma_{w}^{2}=10^{-5}$. Note that the first stage of the observation model is linear, the second stage is nonlinear to the second order, and the third stage is nonlinear to the third order. This will play an important role in the analysis of the algorithms as the EKF is accurate to a first order Taylor series expansion while the UKF is accurate to the second order.

\subsubsection{Calculating the Likelihood and Prior Densities}

The model described in the aforementioned section uses Gaussian noise models. Hence, the likelihood and prior densities are calculated using the Gaussian density function

$$
p(x)=\frac{1}{\sqrt{2 \pi \sigma^{2}}} e^{-\frac{1}{2 \sigma^{2}}\left(x_{s}-x\right)^{2}}
$$

where $\sigma^{2}$ is the variance, $x$ is the current state, and $x_{s}$ is the sample. Shown in Fig 4.1 are Gaussian density functions for zero mean random variables with $\sigma^{2}=$ $\{1,2,3\}$. Notice that as $\sigma^{2}$ increases the width of the bell shape increases and hence the variability of the density also increases. In the context of filtering, the problem of correctly estimating a signal gets harder if the $\sigma^{2}$ value of the Gaussian noise term is larger. In terms of area under the curve, $99.99 \%$ of the area is within 4 standard deviations of the mean.

\subsubsection{Changing the Number of Particles}

In this section the number of particles used in the simulations is varied to determine the effects the number of particles has on the various filters described in chapter 3. Each simulation is run 100 times. A different random seed is used for each simulation. The results are then averaged over the 100 runs in order to determine the RMSE values 


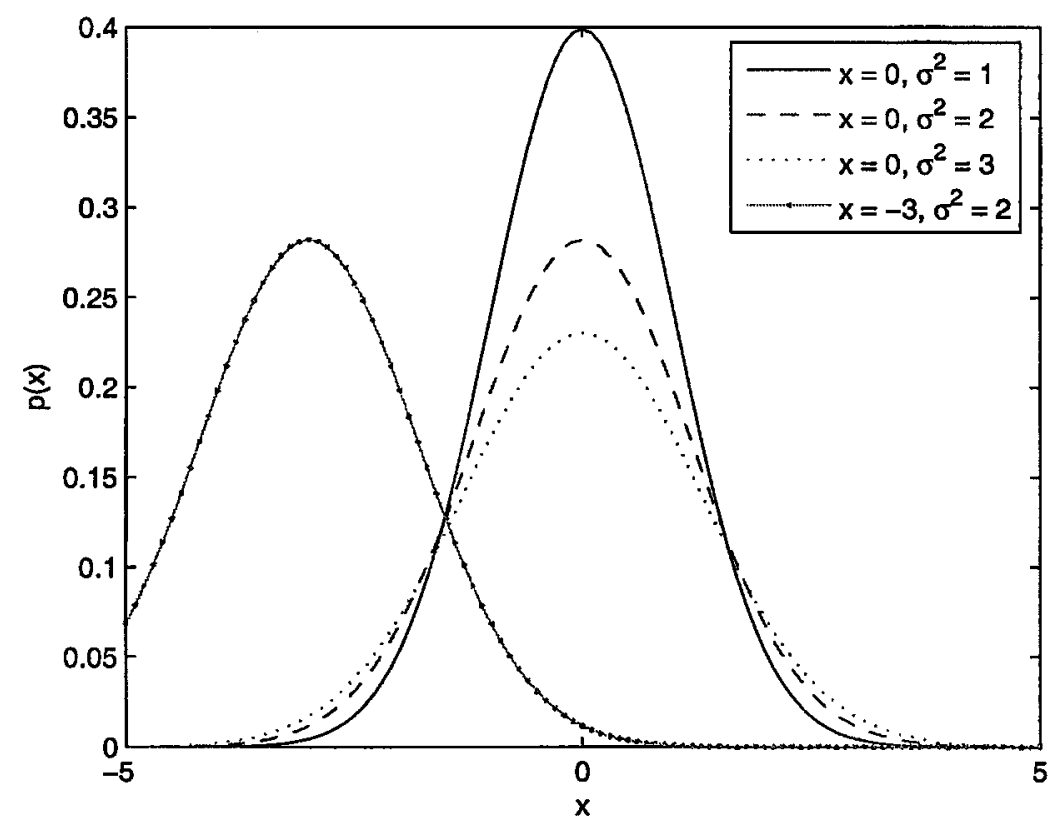

Figure 4.1: Sample distributions for zero mean Gaussian random variables.

of each filter. The variance of the RMSE results over the 100 runs is also calculated for each filter. Each run consists of 60 observations. The results are shown in Table 4.1.

The UKF clearly outperforms the EKF in terms of both RMSE and variance according to Table 4.1. This can be attributed to the fact that the UKF is accurate to the second order Taylor series expansion while the EKF is only accurate to the first order. Of all the filters, the UKF has the lowest variance of RMSE results in this case.

All the results listed in Table 4.1 indicate that an increased number of particles improves the performance of the various particle filters. This is an expected result as more particles allow for a more extensive exploration of the state space. For this particular system model the AEKPF and AUKPF outperform the other particle filters. Besides the case of only 10 particles, the AEKPF and the AUKPF also outperform the UKF in terms of RMSE. 


\begin{tabular}{|l|ccc|ccc|}
\hline & \multicolumn{3}{|c|}{ RMSE } & \multicolumn{3}{c|}{ Variance } \\
\hline Particles & 10 & 100 & 1000 & 10 & 100 & 1000 \\
\hline EKF & 0.69 & 0.69 & 0.69 & 0.057 & 0.057 & 0.057 \\
UKF & 0.55 & 0.55 & 0.55 & 0.020 & 0.020 & 0.020 \\
SIRPF & 1.12 & 0.76 & 0.55 & 0.036 & 0.049 & 0.054 \\
\hline ASIRPF & 1.14 & 0.82 & 0.60 & 0.024 & 0.036 & 0.049 \\
EKPF & 0.73 & 0.60 & 0.55 & 0.057 & 0.086 & 0.066 \\
AEKPF & 0.68 & 0.56 & 0.49 & 0.048 & 0.055 & 0.058 \\
UKPF & 0.69 & 0.57 & 0.51 & 0.036 & 0.079 & 0.067 \\
AUKPF & 0.62 & 0.53 & 0.50 & 0.024 & 0.060 & 0.058 \\
\hline
\end{tabular}

Table 4.1: RMSE and Variance results using 10, 100, and 1000 particles with the additive Gaussian noise model. The model noise is $v_{k} \sim N(0,1)$ while the observation noise is $w_{k} \sim N\left(0,10^{-5}\right)$. The experiment is run 100 times with each trial having 60 observations.

In comparison to the contemporary filters the EKF gets outperformed in almost every simulation. Using only 10 particles, the RMSE results of the EKF are better on average than the SIRPF, ASIRPF, and the EKPF. When using 100 particles the RMSE results of the EKF are better on average than only the SIRPF and the ASIRPF. The AEKPF, UKPF, and the AUKPF perform better than the EKF in all cases.

Plots for one simulation with all the filters running with 100 particles are shown in Fig 4.2-4.5.

\subsubsection{Comparing the Results from the Three Observation Stages}

For this section the simulation was broken into three pieces such that the results of the filters for the various observation stages can be illustrated. The model noise is still Gaussian with $v_{k} \sim N(0,1)$ while the observation noise is $w_{k} \sim N\left(0,10^{-5}\right)$. The experiment is run 100 times with each trial having 20 observations. The results are 


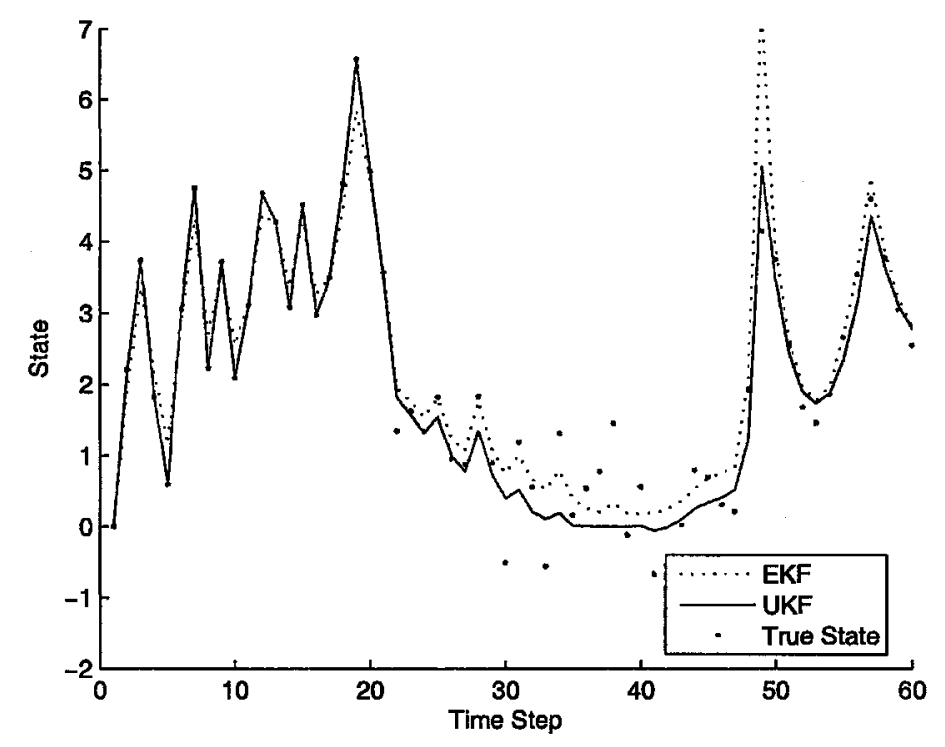

Figure 4.2: EKF and UKF sample runs with Gaussian noise. (RMSE: EKF=0.592, $\mathrm{UKF}=0.411$, Worst Error: $\mathrm{EKF}=3.229, \mathrm{UKF}=1.451$.)

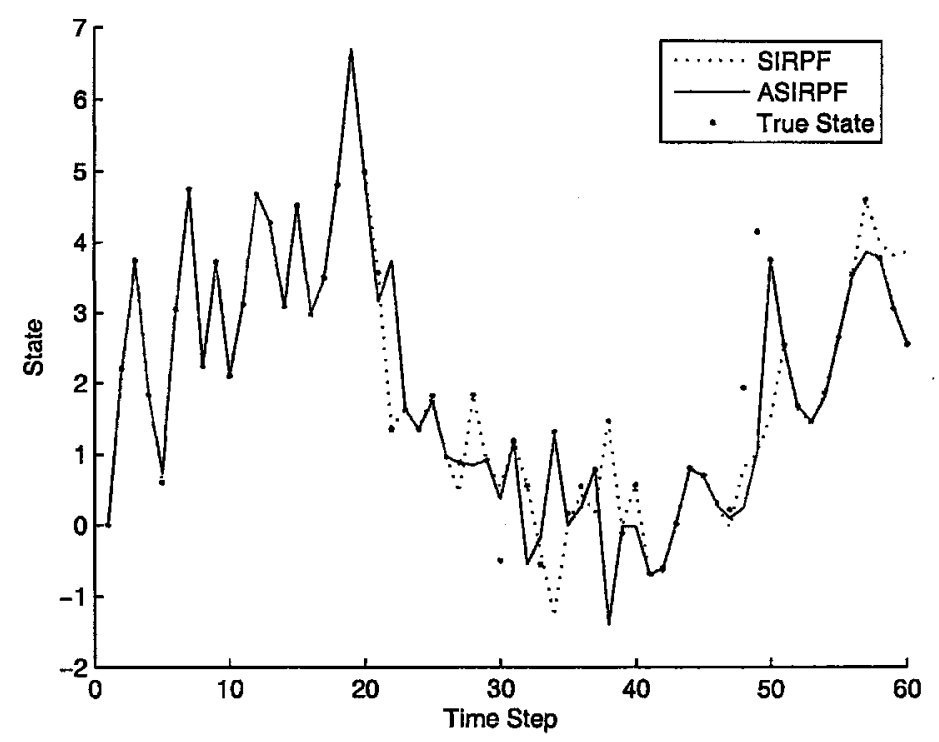

Figure 4.3: SIRPF and ASIRPF sample runs with Gaussian noise. (RMSE: $\mathrm{SIRPF}=0.671, \mathrm{ASIRPF}=0.717$, Worst Error: $\mathrm{SIRPF}=3.125, \mathrm{ASIRPF}=3.122$.) 


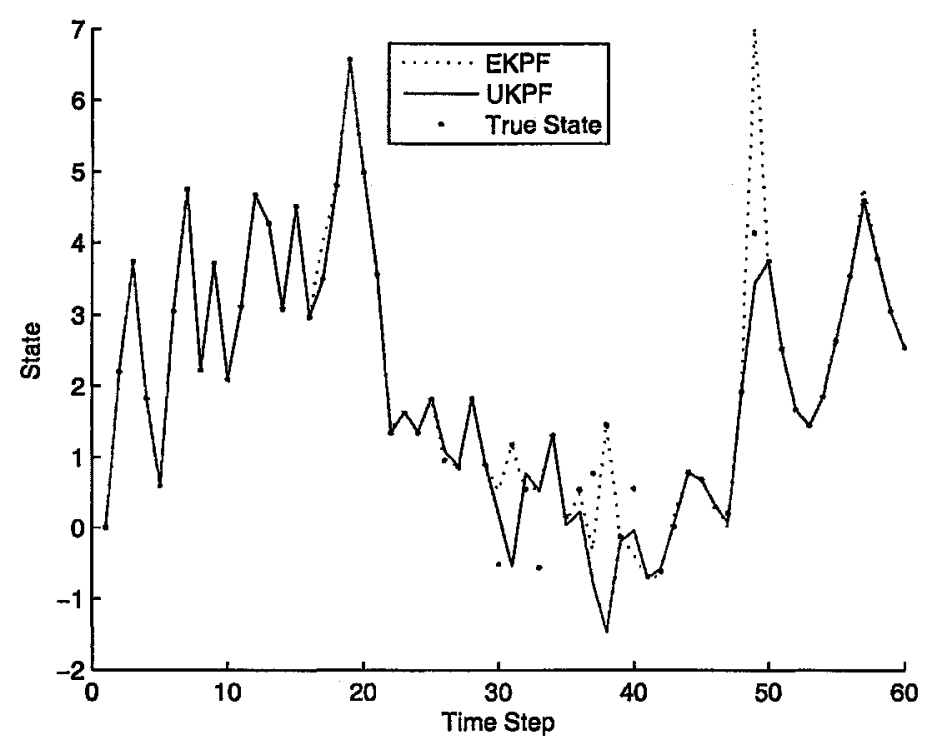

Figure 4.4: EKPF and UKPF sample runs with Gaussian noise. (RMSE: $\mathrm{EKPF}=0.471, \mathrm{UKPF}=0.52678$, Worst Error: $\mathrm{EKPF}=2.909, \mathrm{UKPF}=2.91153$.)

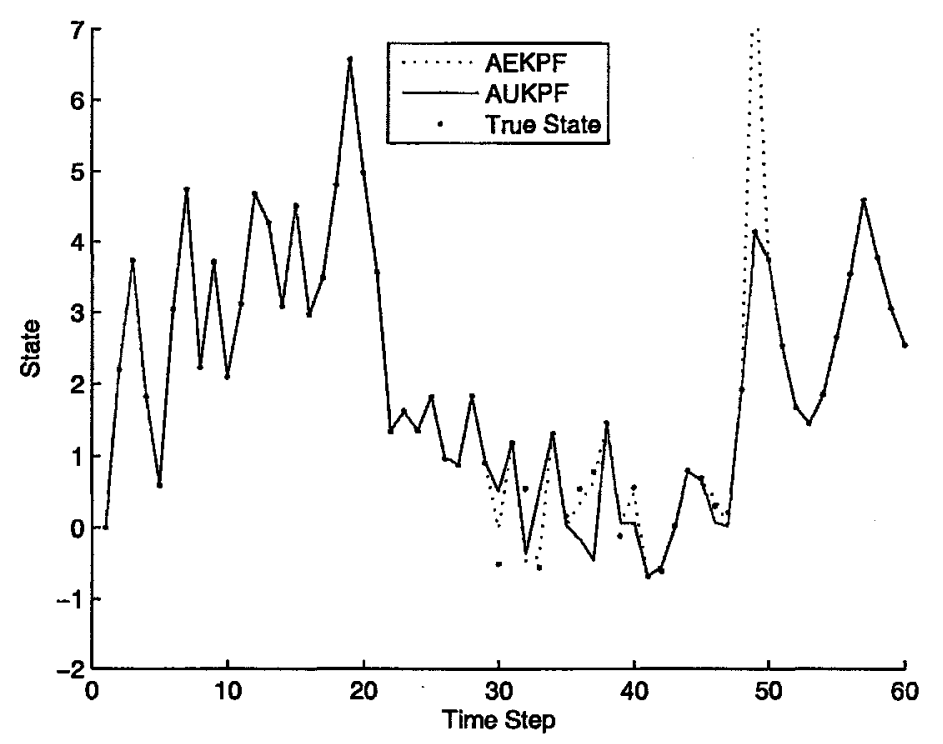

Figure 4.5: AEKPF and AUKPF sample runs with Gaussian noise. (RMSE: $\mathrm{AEKPF}=0.543, \mathrm{AUKPF}=0.30052$, Worst Error: $\mathrm{AEKPF}=4.023, \mathrm{AUKPF}=1.23514$.) 
shown in Table 4.2.

The first point to note is that all the filters perform best in the first stage and worst in the third stage. This is because the degree of nonlinearity is increasing in each stage. As indicated in Eqn 4.2 the first stage of observation is linear, the second stage is nonlinear to the second order, and the third stage is nonlinear to the third order. All the filters except the SIRPF and the ASIRPF achieve similar results in the linear stage. The SIRPF and the ASIRPF perform relatively poorly in the first stage, however, their performance improves when the number of particles increases from 10 to 100 .

In the second stage the advantages of some of the particle filters start to become clear. With only 10 particles the AUKPF outperforms all other filters. With 100 particles, the UKPF and the AUKPF distinguish themselves further from the other filters. Their RMSEs are approximately a fifth of the other filters. The AEKPF also shows promising improvement in the transition from 10 to 100 particles. As in the first stage the SIRPF and ASIRPF are the worst performers again in the second stage. Both their RMSEs and their variances are much higher than all the other filters for both the 10 and 100 particle cases.

The AUKPF performs the best in the third stage. The AUKPF's RMSE is less than half the next best performing filter, the UKPF. In terms of variance, the AUKPF is also clearly the best performing. Excluding the AUKPF, the AEKPF and the UKPF perform much better than the other filters. The RMSEs of the AEKPF and UKPF are approximately half that of the EKF. Again, at only 10 and 100 particles, the SIRPF and the ASIRPF perform the worst, however, they do show much improvement from the 10 particle experiment to the 100 particle experiment.

It is interesting to note that the RMSEs from the three stages analyzed in this section do not average out to the RMSEs found in the experiments of the previous 


\begin{tabular}{|l|cc|cc|cc|}
\hline \multicolumn{7}{|c|}{ RMSE } \\
\hline Stage & \multicolumn{2}{|c|}{ First } & \multicolumn{2}{|c|}{ Second } & \multicolumn{2}{c|}{ Third } \\
\hline Particles & 10 & 100 & 10 & 100 & 10 & 100 \\
\hline EKF & 0.01 & 0.01 & 0.25 & 0.25 & 0.48 & 0.48 \\
UKF & 0.01 & 0.01 & 0.21 & 0.21 & 0.38 & 0.38 \\
SIRPF & 0.97 & 0.27 & 1.07 & 0.53 & 1.10 & 0.71 \\
ASIRPF & 1.01 & 0.41 & 1.07 & 0.65 & 1.08 & 0.77 \\
EKPF & 0.01 & 0.01 & 0.25 & 0.25 & 0.48 & 0.48 \\
AEKPF & 0.01 & 0.01 & 0.23 & 0.16 & 0.44 & 0.27 \\
UKPF & 0.01 & 0.01 & 0.26 & 0.06 & 0.57 & 0.24 \\
AUKPF & 0.01 & 0.01 & 0.15 & 0.04 & 0.37 & 0.10 \\
\hline
\end{tabular}

\begin{tabular}{|l|cc|cc|cc|}
\hline \multicolumn{7}{|c|}{ Variance } \\
\hline Stage & \multicolumn{2}{|c|}{ First } & \multicolumn{2}{c|}{ Second } & \multicolumn{2}{c|}{ Third } \\
\hline Particles & 10 & 100 & 10 & 100 & 10 & 100 \\
\hline EKF & 0.000 & 0.000 & 0.010 & 0.010 & 0.038 & 0.038 \\
UKF & 0.000 & 0.000 & 0.008 & 0.008 & 0.012 & 0.012 \\
SIRPF & 0.060 & 0.080 & 0.045 & 0.081 & 0.052 & 0.077 \\
ASIRPF & 0.056 & 0.094 & 0.056 & 0.077 & 0.051 & 0.074 \\
EKPF & 0.000 & 0.000 & 0.010 & 0.010 & 0.038 & 0.039 \\
AEKPF & 0.000 & 0.000 & 0.011 & 0.010 & 0.041 & 0.012 \\
UKPF & 0.000 & 0.000 & 0.010 & 0.012 & 0.018 & 0.029 \\
AUKPF & 0.000 & 0.000 & 0.008 & 0.008 & 0.007 & 0.005 \\
\hline
\end{tabular}

Table 4.2: RMSE and Variance results using 10 and 100 particles with the additive Gaussian noise model. The simulation was broken into three sections such that the results of the filters for the various observation stages can be illustrated. The model noise is $v_{k} \sim N(0,1)$ while the observation noise is $w_{k} \sim N\left(0,10^{-5}\right)$. The experiment is run 100 times with each trial having 20 observations.

section, Section 4.2.3. In fact, the RMSEs of Section 4.2 .3 are higher. This is because the experiments in the previous section are run with a non-stationary observation equation for 60 observations while the experiments in this section are run independently for each stage of 20 observations. It is assumed that the processes start at an initial state of 0 . Due to the non-stationarity of the observation model in the last section the filters must adapt to the different observation stages over the 60 observations. The experiments in this section each start from an initial known state of 0 and 
only run for 20 observations without any non-stationary element.

\subsubsection{Changing the Noise Variances}

This section examines the filter performances when the values of the system model noise variance $v_{k}$ and observation model noise variance $w_{k}$ are changed. The results are listed in Table 4.3 .

The first experiment tested how well the filters work with a greater noise term added to the observation equation. In this case $\sigma_{v}^{2}=1$ as before, however, $\sigma_{w}^{2}$ was increased from $10^{-5}$ to 1 . The results in Table 4.3 show that increasing the observation noise has a detrimental effect on the particle filters' performances. The UKF and EKF outperform all other filters in the case of 10 particles. Using 100 particles the performance of the SIRPF and the ASIRPF is equal to that of the EKF but all the other particle filters perform worse still. A good observation model is clearly necessary for the particle filters.

The second experiment is similar to the first except the observation model noise was increased further such that $\sigma_{w}^{2}=10$. The results indicate that none of the filters really work much better than the others. Also, increasing the number of particles from 10 to 100 does not really improve the performance of the particle filters.

In the third experiment listed in Table 4.3 the system model noise variance is set such that $\sigma_{v}^{2}=10$. The observation noise was set back to its original variance of $\sigma_{w}^{2}=10^{-5}$. With the noisy system model and accurate observation model the AUKPF proves to be the best performing filter in terms or RMSE. The UKPF also faired quite well under these test conditions. Furthermore, the UKF worked better than the EKF again. Increasing the number of particles in this test case reduced the RMSE of the particle filters. The RMSE results of all the particle filters was less than that of the EKF RMSE. 


\begin{tabular}{|l|cc|cc|cc|}
\hline Error & \multicolumn{2}{|c|}{$\sigma_{v}^{2}=1, \sigma_{w}^{2}=1$} & \multicolumn{2}{|c|}{$\sigma_{v}^{2}=1, \sigma_{w}^{2}=10$} & \multicolumn{2}{c|}{$\sigma_{v}^{2}=10, \sigma_{w}^{2}=10^{-5}$} \\
\hline Particles & 10 & 100 & 10 & 100 & 10 & 100 \\
\hline EKF & 0.92 & 0.92 & 1.04 & 1.04 & 15.66 & 15.66 \\
UKF & 0.89 & 0.89 & 1.05 & 1.05 & 9.27 & 9.27 \\
SIRPF & 1.00 & 0.92 & 1.13 & 1.06 & 11.95 & 10.75 \\
ASIRPF & 1.00 & 0.91 & 1.12 & 1.05 & 11.86 & 11.25 \\
EKPF & 1.15 & 1.44 & 1.14 & 1.24 & 13.83 & 13.63 \\
AEKPF & 1.00 & 1.01 & 1.06 & 1.06 & 14.29 & 12.85 \\
UKPF & 1.15 & 1.45 & 1.12 & 1.27 & 9.59 & 9.80 \\
AUKPF & 1.00 & 1.02 & 1.05 & 1.06 & 8.77 & 7.87 \\
\hline
\end{tabular}

Table 4.3: RMSE results using 10 and 100 particles with the additive Gaussian noise model. The simulations are conducted with various noise levels.

From the results it appears that the particle filters' strengths are estimation of the system state when the system model is noisy and the observation model is accurate. The next section will examine the effects of changing the noise distributions.

\subsection{Gamma Noise Model Results}

\subsubsection{Model Description}

For this experiment, the nonlinear process model is as follows

$$
x_{k}=1+\sin (\omega \pi k)+\phi_{1} x_{k}+v_{k}
$$

where $\omega=0.04$ and $\phi_{1}=0.5$ are scalar parameters and $v_{k}$ is a $\operatorname{Gamma,} \mathrm{Ga}(3,2)$, random variable modeling the process noise. The non-stationary element of the ex- 
periment is present in the observation model

$$
y_{k}= \begin{cases}\phi_{2} x_{k}-2+w_{k} & (k \leq 20) \\ \phi_{3} x_{k}^{2}+w_{k} & (20<k \leq 40) \\ \phi_{4} x_{k}^{3}+w_{k} & (k>40)\end{cases}
$$

where $\phi_{2}=0.2, \phi_{3}=0.5$, and $\phi_{4}=0.1$ are scalar parameters and $w_{k}$, the observation noise, is modelled as a Gaussian random variable, $w_{k} \sim N\left(0, \sigma_{w}^{2}\right)$, where $\sigma_{w}^{2}=10^{-5}$. This is a particularly interesting case as the Gamma noise term violates the additive Gaussian noise assumptions required for the EKF and the UKF.

\subsubsection{Calculating the Likelihood and Prior Densities}

The model described in the aforementioned section uses a Gamma random variable to model process noise. Although, the likelihood densities are calculated using the Gaussian density function, the prior distribution is calculated using the Gamma density function

$$
p(x)= \begin{cases}\frac{\beta^{\alpha+1}}{\gamma(\alpha+1)} x^{\alpha} \exp ^{-\beta x} & (x \geq 0) \\ 0 & (x<0)\end{cases}
$$

where $\alpha$ and $\beta$ are parameters describing the function, i.e., $\sim G a(\alpha, \beta)$. Gamma distributions for various parameter selections are shown in Fig 4.6.

One of the main differences between the Gamma and Gaussian distributions is that the Gamma distribution is not symmetric. As is seen in Fig 4.6 sampling from the Gamma distribution only produces positive numbers. The results for filtering the system with additive Gamma noise are described in the next section. 


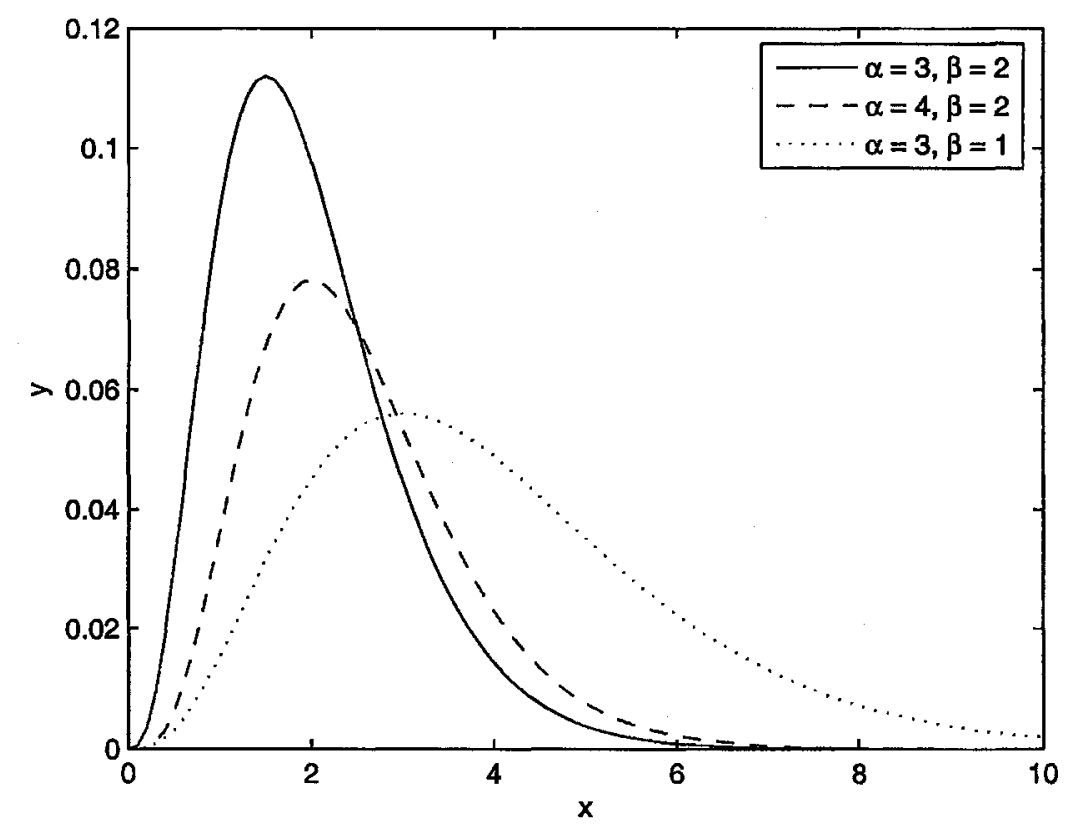

Figure 4.6: Sample distributions for Gamma random variables.

\subsubsection{Gamma Noise Results}

The modern UKF outperforms the EKF again in the Gamma noise experiment. This is true in terms of both RMSE and variance as shown in Table 4.4. Similarly, the best overall filter is again the AUKPF. It is the best overall filter with only 10 particles. At 100 particles it is even better, with a RMSE that is about one third of that of the UKF, and about a fifth of the the EKF RMSE. Although its performance was merely average with 10 particles, the AEKPF worked relatively well with 100 particles. With 100 particles the AEKPF's RMSE results were almost as low as the AUKPF. The worst performing particle filter was the EKPF. Its RMSE results were similar to those of the EKF.

Comparing the results of the Gamma noise experiments to the Gaussian noise experiments it is evident that the UKF and especially the EKF performed worse 


\begin{tabular}{|l|cc|cc|}
\hline & \multicolumn{2}{|c|}{ RMSE } & \multicolumn{2}{c|}{ Variance } \\
\hline Particles & 10 & 100 & 10 & 100 \\
\hline EKF & 1.228 & 1.228 & 0.716 & 0.716 \\
\hline UKF & 0.728 & 0.728 & 0.217 & 0.217 \\
SIRPF & 0.962 & 0.651 & 0.029 & 0.041 \\
ASIRPF & 0.974 & 0.742 & 0.026 & 0.035 \\
EKPF & 1.244 & 1.158 & 0.856 & 0.886 \\
AEKPF & 0.854 & 0.297 & 0.483 & 0.173 \\
UKPF & 0.854 & 0.571 & 0.458 & 0.192 \\
AUKPF & 0.558 & 0.238 & 0.134 & 0.156 \\
\hline
\end{tabular}

Table 4.4: RMSE and Variance results using 10 and 100 particles with the additive Gamma noise model.

while trying to estimate the signals corrupted by the Gamma noise. Conversely, most of the particle filters performed better in the case with the Gamma noise. The main exception being the EKPF whose results closely match those of the EKF.

Plots for one simulation with all the filters running with 100 particles are shown in Fig 4.7-4.10.

\subsection{Cauchy Noise Model Results}

\subsubsection{Model Description}

For this experiment, the nonlinear process model is as follows

$$
x_{k}=1+\sin (\omega \pi k)+\phi_{1} x_{k}+v_{k}
$$

where $\omega=0.04$ and $\phi_{1}=0.5$ are scalar parameters and $v_{k}$ is a Cauchy, $\mathrm{Ca}(0,0.1)$, random variable modeling the process noise. The non-stationary element of the ex- 


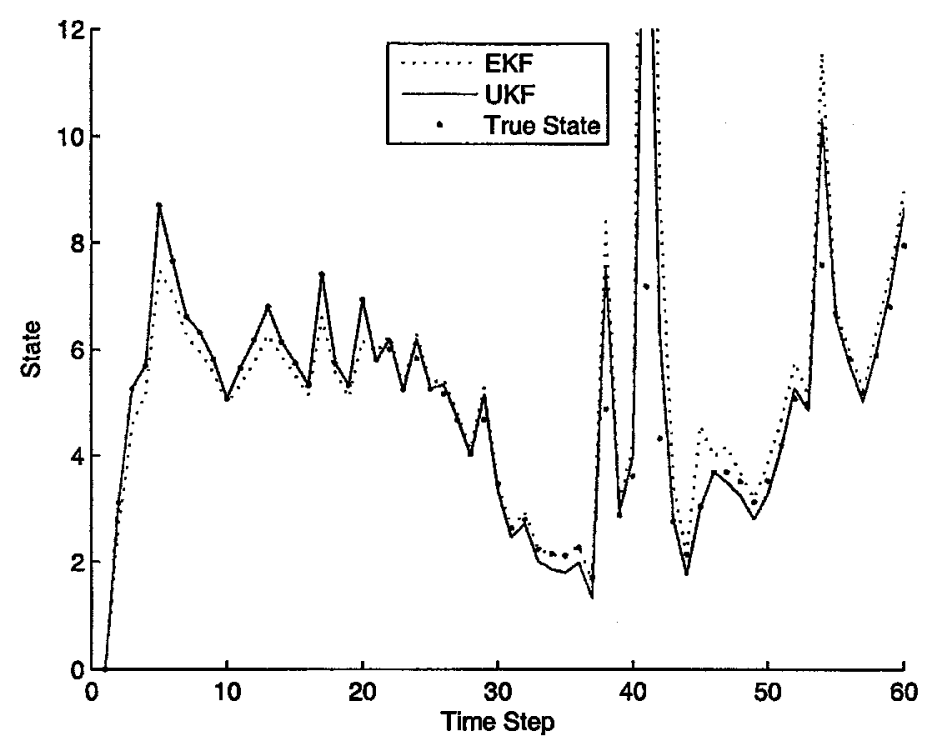

Figure 4.7: EKF and UKF sample runs with Gamma noise. (RMSE: EKF=2.614, $\mathrm{UKF}=1.444$, Worst Error: $\mathrm{EKF}=18.672, \mathrm{UKF}=10.219$.)

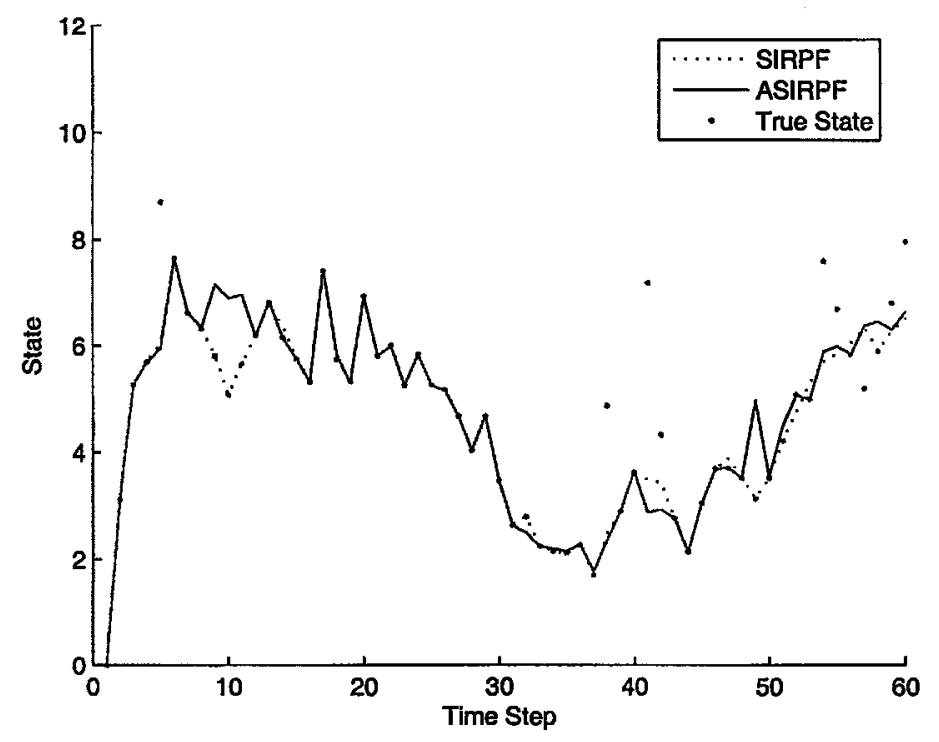

Figure 4.8: SIRPF and ASIRPF sample runs with Gamma noise. (RMSE: $\mathrm{SIRPF}=0.770, \mathrm{ASIRPF}=0.931$, Worst Error: $\mathrm{SIRPF}=3.696, \mathrm{ASIRPF}=4.306$.) 


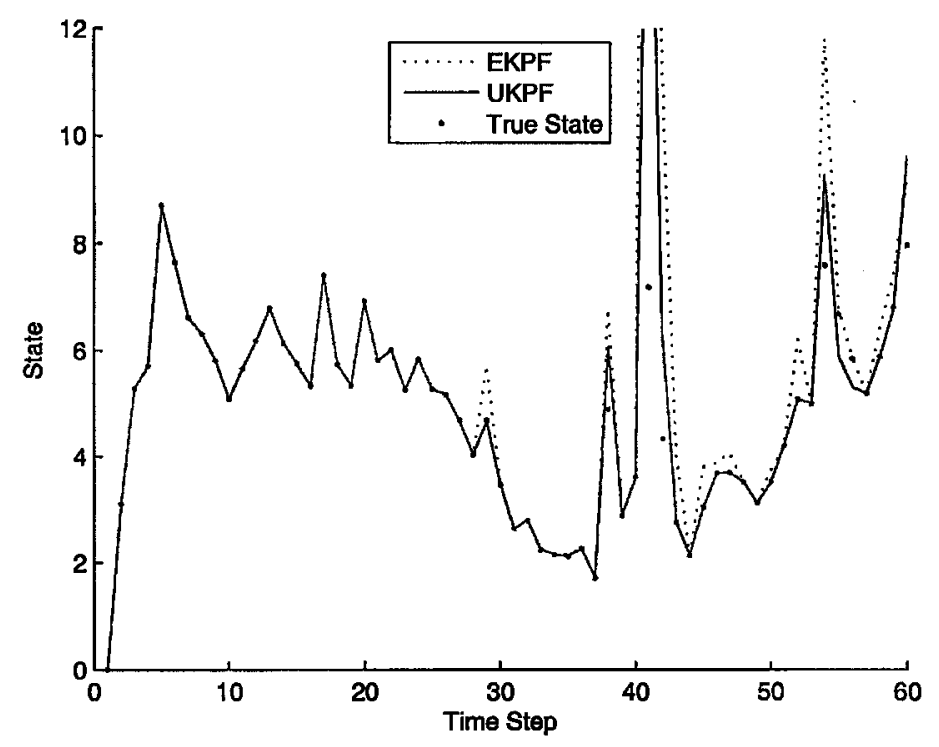

Figure 4.9: EKPF and UKPF sample runs with Gamma noise. (RMSE: EKPF=3.466, $\mathrm{UKPF}=1.50084$, Worst Error: $\mathrm{EKPF}=25.415, \mathrm{UKPF}=11.11560$.)

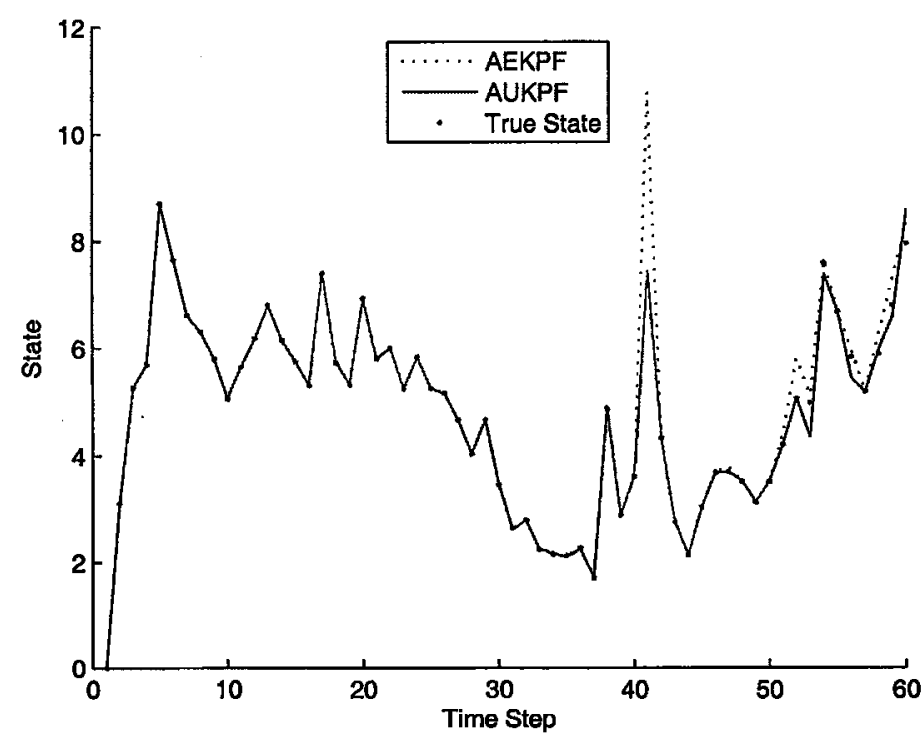

Figure 4.10: AEKPF and AUKPF sample runs with Gamma noise. (RMSE: $\mathrm{AEKPF}=0.488, \mathrm{AUKPF}=0.13746$, Worst Error: $\mathrm{AEKPF}=3.614, \mathrm{AUKPF}=0.63926$.) 
periment is present in the observation model

$$
y_{k}= \begin{cases}\phi_{2} x_{k}-2+w_{k} & (k \leq 20) \\ \phi_{3} x_{k}^{2}+w_{k} & (20<k \leq 40) \\ \phi_{4} x_{k}^{3}+w_{k} & (k>40)\end{cases}
$$

where $\phi_{2}=0.2, \phi_{3}=0.5$, and $\phi_{4}=0.1$ are scalar parameters and $w_{k}$, the observation noise, is modelled as a Gaussian random variable, $w_{k} \sim N\left(0, \sigma_{w}^{2}\right)$, where $\sigma_{w}^{2}=10^{-5}$. Again, this is an interesting case as the Cauchy noise term violates the additive Gaussian noise assumptions required for the EKF and the UKF.

\subsubsection{Calculating the Likelihood and Prior Densities}

The model described in the aforementioned section uses a Cauchy random variable to model process noise. Although, the likelihood densities are calculated using the Gaussian density function, the prior distribution is calculated using the Cauchy density function

$$
p(x)=\frac{1}{\pi \gamma\left[1+\left(\frac{x-x_{0}}{\gamma}\right)^{2}\right]}
$$

where $x_{0}$ and $\gamma$ are parameters describing the function, i.e., $\sim C a\left(x_{0}, \gamma\right)$. Cauchy distributions for various parameter selections are shown in Fig 4.11.

The Cauchy distribution is similar in appearance to the Gaussian distribution. The main difference is the size of the distribution's tails. The Cauchy distribution has larger tails than a Gaussian distribution meaning that there is a higher probability of an outlier occuring when sampling from the Cauchy distribution. 


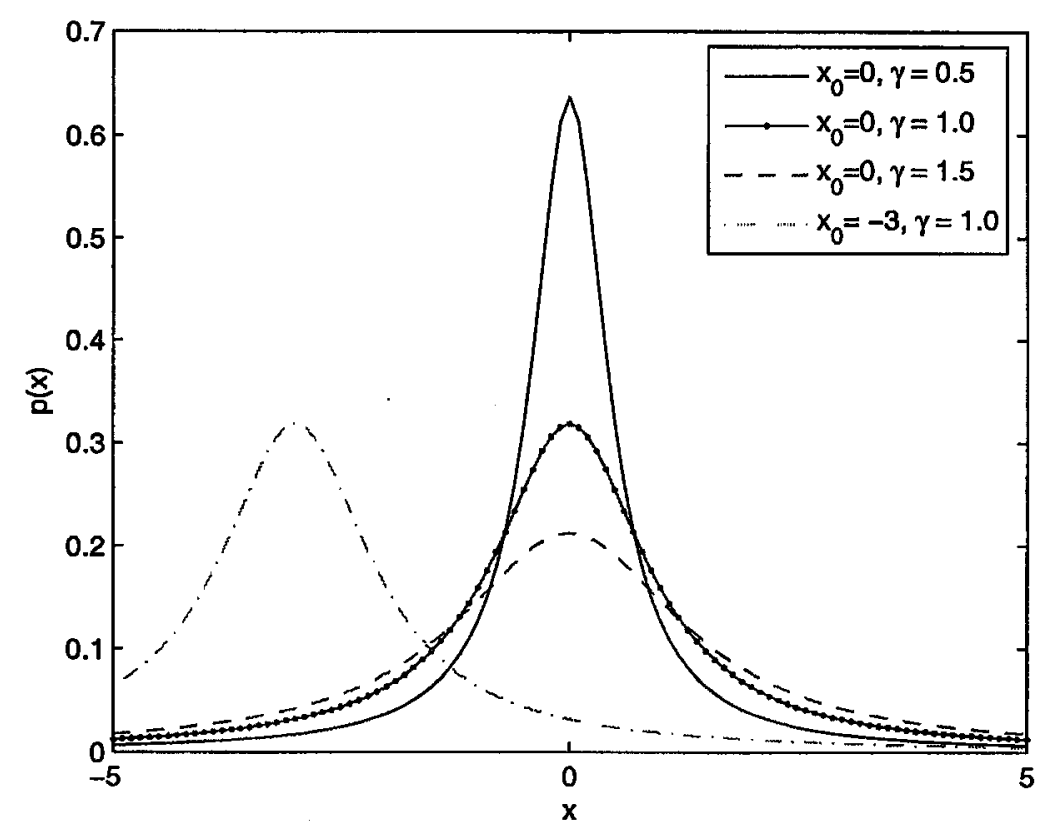

Figure 4.11: Sample distributions for Cauchy random variables.

\subsubsection{Cauchy Noise Results}

The first experiment conducted with the Cauchy noise term was to test how the filters performed with 10 and 100 particles using the non-stationary observation model for 60 observations. The results are shown in Table 4.5.

The first thing to note is that the performance of the filters is noticeably worse than in any of the previous experiments. The EKF has extremely poor results with a RMSE of 81.04. The particle filters based on the EKF also have poor results. The UKF results are much better relative to the EKF. Similarly, the UKF based particle filters also prove to have better RMSE results.

The best performances were achieved by the SIRPF and the ASIRPF. They outperformed the other filters in terms of RMSE and variance. This may be explained by the fact that the prior is used as the proposal function for the SIRPF and the 


\begin{tabular}{|l|cc|cc|}
\hline & \multicolumn{2}{|c|}{ RMSE } & \multicolumn{2}{c|}{ Variance } \\
\hline Particles & 10 & 100 & 10 & 100 \\
\hline EKF & 81.04 & 81.04 & 98665.38 & 98665.38 \\
UKF & 3.91 & 3.91 & 220.28 & 220.28 \\
SIRPF & 2.83 & 2.27 & 22.11 & 21.25 \\
ASIRPF & 4.08 & 2.29 & 40.08 & 16.81 \\
EKPF & 91.14 & 147.37 & 115305.96 & 326864.36 \\
AEKPF & 115.79 & 74.34 & 228114.76 & 90706.25 \\
UKPF & 5.01 & 4.05 & 258.37 & 204.02 \\
AUKPF & 4.54 & 3.85 & 112.33 & 179.30 \\
\hline
\end{tabular}

Table 4.5: RMSE and Variance results using 10 and 100 particles with the additive Cauchy noise model.

ASIRPF. Since the prior is based entirely on the transition model which in this case includes the Cauchy noise term, the SIRPF and the ASIRPF seem to be the best equipped to deal with this system model. The other particle filters use the UKF and EKF to generate proposal functions. Since the EKF and the UKF perform poorly for this system model, so do the EKPF, AEKPF, UKPF, and the AUKPF.

To better understand where the filters break down in the previous simulations another experiment was run isolating each of the 3 observation stages. These simulations are similar to the ones conducted on the Gaussian transition model of Section 4.2.4. The results are listed in Table 4.6 .

The results of the filters are best in the first stage where the observation model is linear, and worst in the third stage where the observation model is third order. In almost all cases increasing the number of particles from 10 to 100 improves the particle filters' performances. This is generally true except for in the first stage where for most filters increasing the number of particles has no significant effect. As the results have shown previously, the UKF outperforms the EKF in each of the three stages both in terms of RMSE and variance.

The results in the first stage where the observation equation is linear are similar to 


\begin{tabular}{|l|cc|cc|cc|}
\hline \multicolumn{9}{|c|}{ RMSE } \\
\hline Stage & \multicolumn{2}{|c|}{ First } & \multicolumn{2}{c|}{ Second } & \multicolumn{2}{c|}{ Third } \\
\hline Particles & 10 & 100 & 10 & 100 & 10 & 100 \\
\hline EKF & 0.01 & 0.01 & 14.14 & 14.14 & 431.08 & 431.08 \\
UKF & 0.01 & 0.01 & 4.95 & 4.95 & 64.09 & 64.09 \\
SIRPF & 1.75 & 1.25 & 1.92 & 1.36 & 2.76 & 1.38 \\
ASIRPF & 1.65 & 1.34 & 1.76 & 1.56 & 1.99 & 1.56 \\
EKPF & 0.01 & 0.01 & 14.14 & 14.14 & 430.60 & 431.95 \\
AEKPF & 0.01 & 0.01 & 14.10 & 9.62 & 431.04 & 443.19 \\
UKPF & 0.01 & 0.01 & 8.30 & 7.22 & 44.52 & 52.46 \\
AUKPF & 0.01 & 0.01 & 7.28 & 4.88 & 63.85 & 10.95 \\
\hline
\end{tabular}

\begin{tabular}{|l|cc|cc|cc|}
\hline \multicolumn{8}{|c|}{ Variance } \\
\hline Stage & \multicolumn{2}{|c|}{ First } & \multicolumn{2}{c|}{ Second } & \multicolumn{2}{c|}{ Third } \\
\hline Particles & 10 & 100 & 10 & 100 & 10 & 100 \\
\hline EKF & 0.0 & 0.0 & 11738.8 & 11738.8 & 15293729.8 & 15293729.8 \\
UKF & 0.0 & 0.0 & 332.0 & 332.0 & 285506.0 & 285506.0 \\
SIRPF & 22.7 & 22.0 & 22.8 & 22.1 & 57.7 & 21.8 \\
ASIRPF & 21.0 & 22.2 & 20.8 & 22.1 & 21.7 & 22.0 \\
EKPF & 0.0 & 0.0 & 11738.8 & 11741.3 & 15295458.5 & 15315776.8 \\
AEKPF & 0.0 & 0.0 & 11738.0 & 6564.5 & 15300026.1 & 15349771.1 \\
UKPF & 0.0 & 0.0 & 756.1 & 588.6 & 109974.0 & 195820.2 \\
AUKPF & 0.0 & 0.0 & 416.9 & 18.8 & 280536.1 & 3463.6 \\
\hline
\end{tabular}

Table 4.6: RMSE and Variance results using 10 and 100 particles with the additive Cauchy noise model. The simulation was broken into three sections such that the results of the filters for the various observation stages can be illustrated.

when the transition model noise term was Gaussian. It is the second and third stages where things are evidently much different. In each of the second and third stages the SIRPF and the ASIRPF outperform the other filters by a relatively large margin in terms of both RMSE and variance. The Cauchy noise term has a detrimental affect when the observation models are not linear. This is especially true in the third case when the observation term is third order. Out of the EKF and UKF based particle filters the AUKPF performs the best in all three stages. The results of the SIRPF and the ASIRPF, however, are still much better than those of the AUKPF. 


\subsubsection{Comparing Single Runs of the Cauchy Noise Simula-} tions

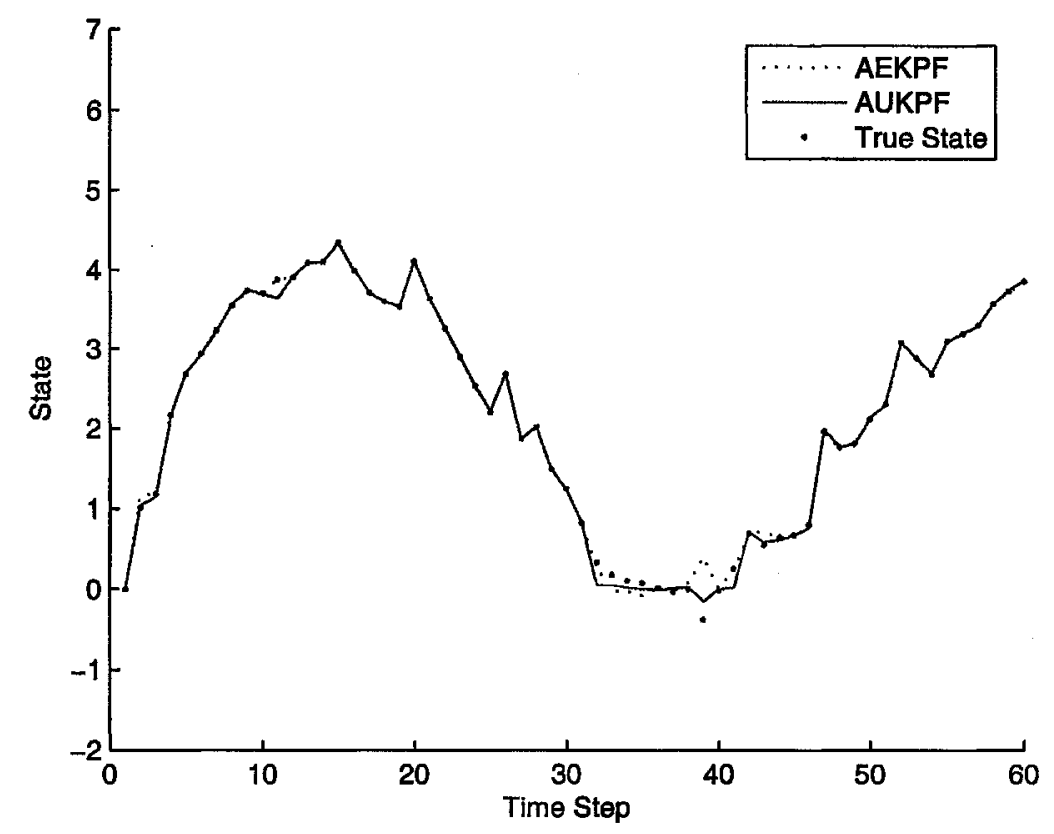

Figure 4.12: AEKPF and AUKPF sample runs with Cauchy noise. (RMSE: $\mathrm{AEKPF}=0.110, \mathrm{AUKPF}=0.06725$, Worst Error: $\mathrm{AEKPF}=0.769, \mathrm{AUKPF}=0.27772$.)

In this section sample simulations conducted with the Cauchy noise model are examined to illustrate the high variance in the results of Table 4.5. The first simulation shown in Fig 4.12 illustrates the AEKPF and the AUKPF performing well while estimating the state of the model with Cauchy noise. The transition model noise term was $\sim C a(0,0.1)$ and the observation model noise term was $\sim N(0,0.00001)$. Their RMSEs in this case are relatively low in this trial run. The AEKPF's RMSE was 0.110, while the AUKPF's RMSE was 0.0673. Furthermore, their worst prediction errors were 0.769 and 0.278 respectively.

The second simulation shown in Fig 4.13 illustrates how the large tails of the Cauchy distribution can have a detrimental effect on the filtering results. Again, the 


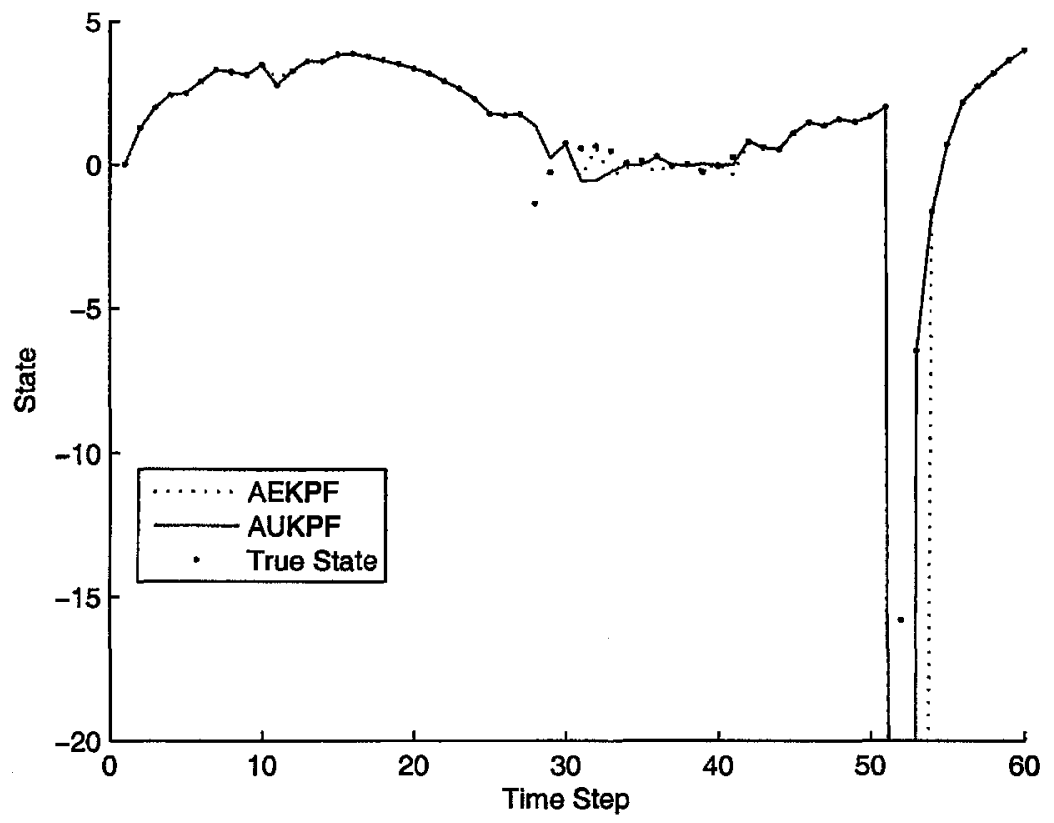

Figure 4.13: AEKPF and AUKPF sample runs with Cauchy noise. (RMSE: $\mathrm{AEKPF}=30.402, \mathrm{AUKPF}=16.25811$, Worst Error: $\mathrm{AEKPF}=224.754$, $\mathrm{AUKPF}=125.89082$.)

transition model noise term was $\sim C a(0,0.1)$ and the observation model noise term was $\sim N(0,0.00001)$. At time step 52 there is a large error in the transition model data due to the Cauchy noise model. As is shown, the AEKPF and the AUKPF do not calculate good state estimates at this time step. In this trial run the AEKPF RMSE was 30.402 and the AUKPF RMSE was 16.258. These large RMSEs are mostly due to the poor estimates at time step 52. The worst prediction errors were 224.754 and 125.890 respectively.

The third simulation shown in Fig 4.14 depicts why the SIRPF and the ASIRPF have better RMSE results for the Cauchy noise simulations. Again, the transition model noise term was $\sim C a(0,0.1)$ and the observation model noise term was $~$ $N(0,0.00001)$. At time step 52 when the true state deviates due to the large error 


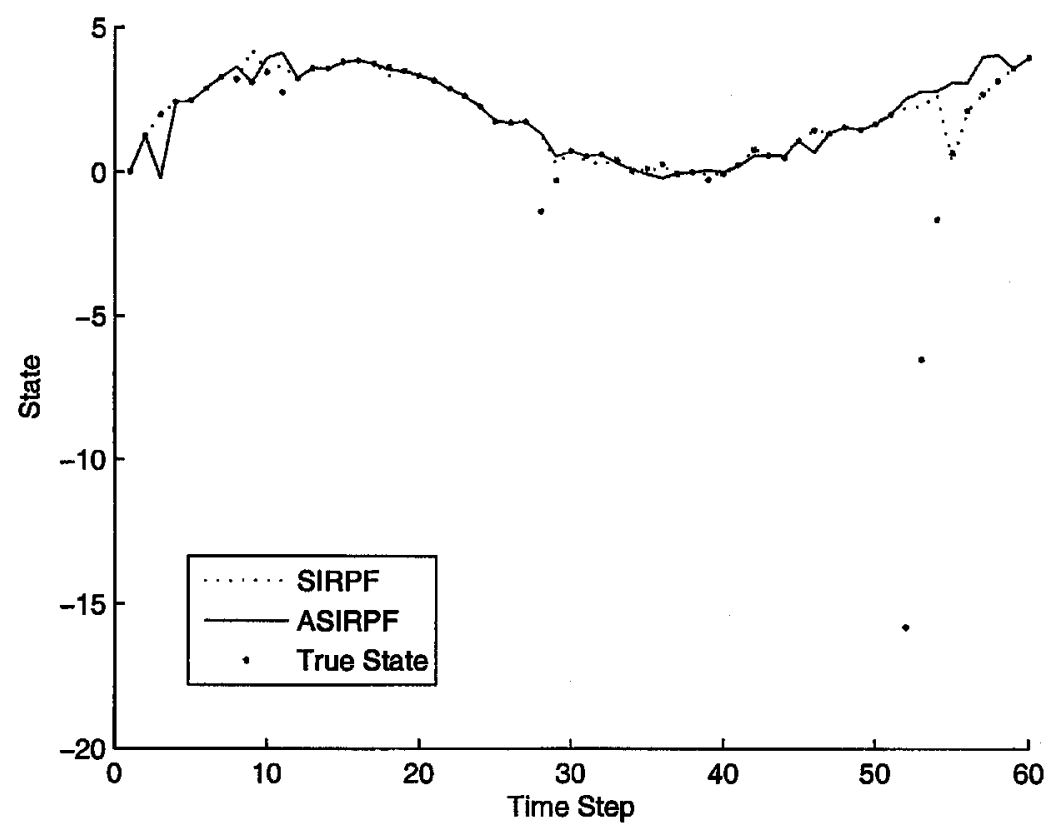

Figure 4.14: SIRPF and ASIRPF sample runs with Cauchy noise. (RMSE: $\mathrm{SIRPF}=2.686, \mathrm{ASIRPF}=2.798$, Worst Error: $\mathrm{SIRPF}=18.069, \mathrm{ASIRPF}=18.367$.)

term the SIRPF and the ASIRPF fail to react. In doing so they accumulate large errors in state estimation, however, these errors are less than those generated by the overcompensation of the other filters. In this case, the RMSE of the SIRPF was only 2.686 while the RMSE of the ASIRPF was only 2.798. Their worst errors were at time step 52 and were 18.069 and 18.367 respectively.

\subsection{Chapter Summary}

This chapter examined a series of simulations conducted with different parameters and noise models to determine the strengths and weaknesses of the various filters introduced in the preceding chapter. The main metric used to determine filter performance was RMSE. The first series of tests was run with a Gaussian noise term in the 
transition model. The number of particles used per simulation was varied, also the variance of the noise terms was also changed. The results of observation models with different degrees of nonlinearity was also presented. The second set of simulations was run on a transition model with a Gamma noise term. The performance of the filters with various numbers of particles was examined. Finally, the third series of simulations was run on a transition model with Cauchy noise added to it. The effects of the large tails associated with the Cauchy noise distribution are observed. 


\section{Chapter 5}

\section{Sample Applications: Simulation and Results}

\subsection{Stochastic Volatility Model}

\subsubsection{Introduction}

Time series analysis and nonlinear filtering are topics of great interest in the fields of finance and econometrics. One such application is estimating measures of financial asset volatility. For instance, the price of a contingent asset such as an option can be modelled such that it depends on the volatility of the original asset. This section concentrates on the discrete time stochastic volatility model, in which the volatility is modelled as a latent stochastic process $[9,20,29,45,58]$. 


\subsubsection{Model Description}

An example of the stochastic volatility model is as follows

$$
\begin{aligned}
x_{t+1} & =\phi_{0}+\phi_{1} x_{t}+\sigma \eta_{t} \\
y_{t} & =e^{\frac{x_{t}}{2}} \varepsilon_{t}
\end{aligned}
$$

for $t=1,2,3, \ldots, T$. In this case, $x_{t}$ represents the stochastic volatility process while $y_{t}$ represents the observation process. The noise terms $\varepsilon_{t}$ and $\eta_{t}$ are Gaussian with zero means and variances of 1 and $\sigma^{2}$ respectively.

Of particular interest is the likelihood calculation associated with this model. It is different than the previous examples because of the dependency on $x_{t}$ in the variance. The derivation is as follows

$$
\begin{aligned}
p\left(y_{t+1} \mid x_{t}\right) & =\frac{1}{\left(2 \pi e^{x_{t}}\right)^{1 / 2}} e^{\frac{-1}{2 e^{2} t}\left(y_{t+1}-0\right)^{2}} \\
& =\frac{1}{(2 \pi)^{1 / 2}} e^{\frac{-x_{t}}{2}} e^{\frac{-1}{2} e^{-x_{t}} y_{t+1}^{2}} \\
& =\frac{1}{(2 \pi)^{1 / 2}} e^{-\frac{1}{2}\left[e^{-x_{t}} y_{t+1}^{2}+x_{t}\right]}
\end{aligned}
$$

\subsubsection{Simulation and Results}

For the simulations conducted with the stochastic volatility model the parameters were set at $\phi_{0}=0.1, \phi_{1}=0.9$, and $\sigma^{2}=1$. Table 5.1 lists the RMSE and variance results for the various filters. The data was collected over 100 independent simulations. The case for 10 and 100 particles are shown.

The results shown in Table 5.1 indicate that there is not much of an advantage using 100 particles over just 10 particles in this case. Only the AEKPF and AUKPF 


\begin{tabular}{|c|c|c|c|c|}
\hline & \multicolumn{2}{|c|}{$\overline{\mathrm{RMSE}}$} & \multicolumn{2}{|c|}{ Variance } \\
\hline Particles & 10 & 100 & 10 & 100 \\
\hline EKF & 1.479 & 1.479 & 0.395 & 0.395 \\
\hline UKF & 1.424 & 1.424 & 0.397 & 0.397 \\
\hline SIRPF & 2.908 & 2.961 & 0.191 & 0.152 \\
\hline ASIRPF & 2.645 & 2.744 & 0.241 & 0.167 \\
\hline EKPF & 1.56 & 1.657 & 0.304 & 0.237 \\
\hline AEKPF & 1.468 & 1.46 & 0.26 & 0.2 \\
\hline UKPF & 1.527 & 1.632 & 0.304 & 0.232 \\
\hline AUKPF & 1.395 & 1.383 & 0.165 & 0.141 \\
\hline
\end{tabular}

Table 5.1: RMSE and Variance results using 10 and 100 particles with stochastic volatility model.

show improvement with 100 particles. In this case it is also shown that the particle filters offer little improvement on the results of the EKF and UKF filters. In fact, again only the AEKPF and the AUKPF have lower RMSE values than the EKF and UKF filters.

The results from a single simulation run are illustrated in Figures 5.1-5.4. For this example trial the AEKPF and AUKPF are clearly the best performing filters. They achieved the lowest RMSE values as well as the lowest worst case error values. 


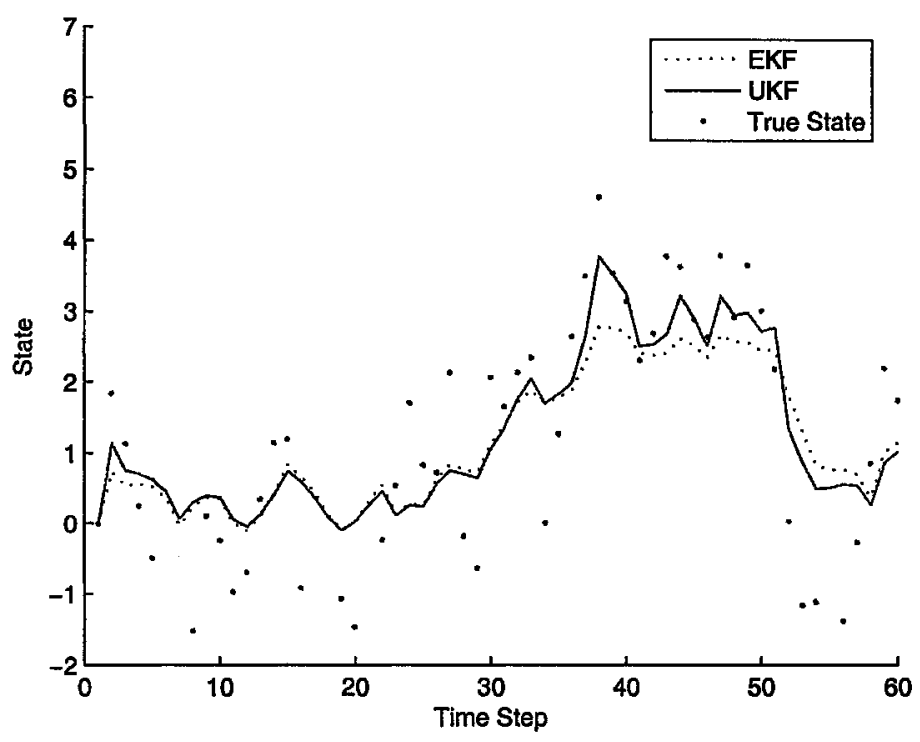

Figure 5.1: EKF and UKF sample runs with the stochastic volatility model. (RMSE: $\mathrm{EKF}=1.32, \mathrm{UKF}=1.22$, Worst Error: $\mathrm{EKF}=3.10, \mathrm{UKF}=3.18$.)

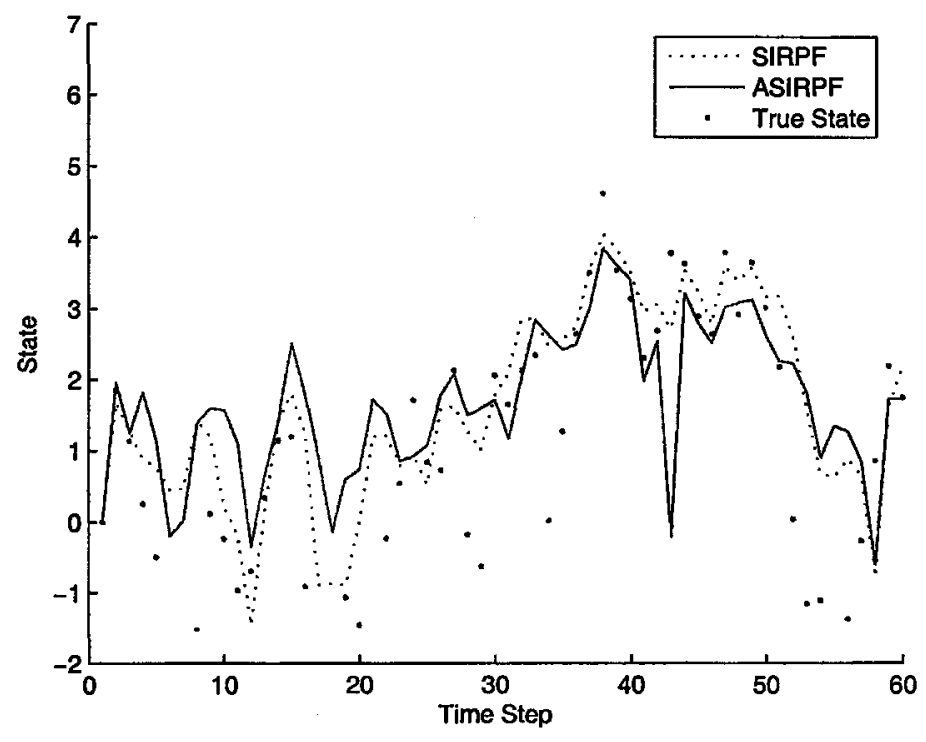

Figure 5.2: SIR and ASIR sample runs with the stochastic volatility model. (RMSE: $\mathrm{SIR}=1.36, \mathrm{ASIR}=1.66$, Worst Error: $\mathrm{SIR}=3.27, \mathrm{ASIR}=3.99$.) 


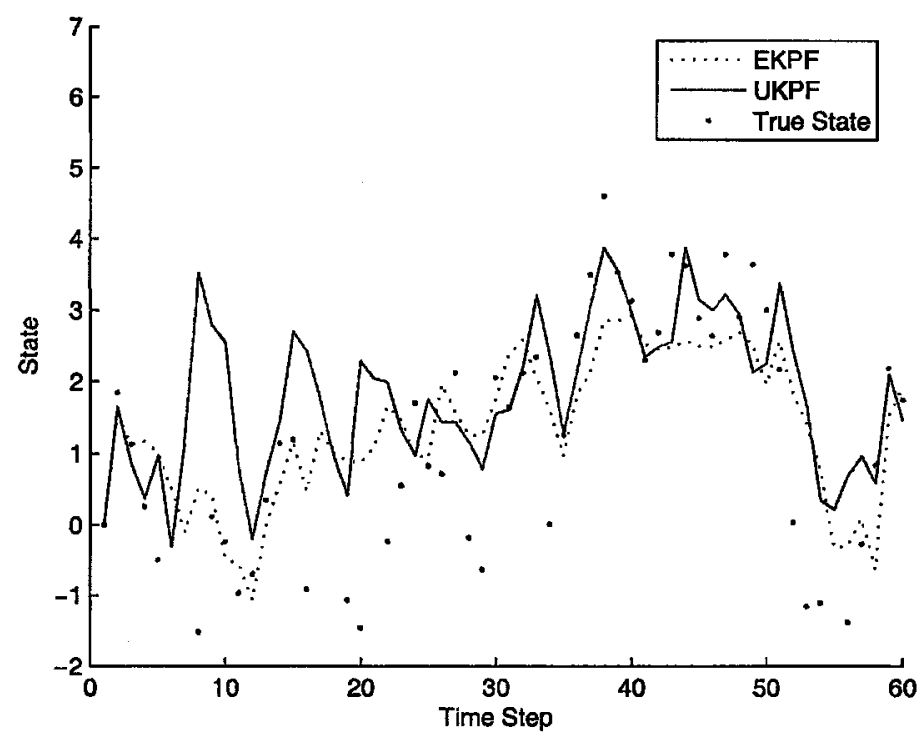

Figure 5.3: EKPF and UKPF sample runs with the stochastic volatility model. (RMSE: $\mathrm{EKPF}=1.42, \mathrm{UKPF}=1.81$, Worst Error: $\mathrm{EKPK}=3.97, \mathrm{UKPF}=5.04$.)

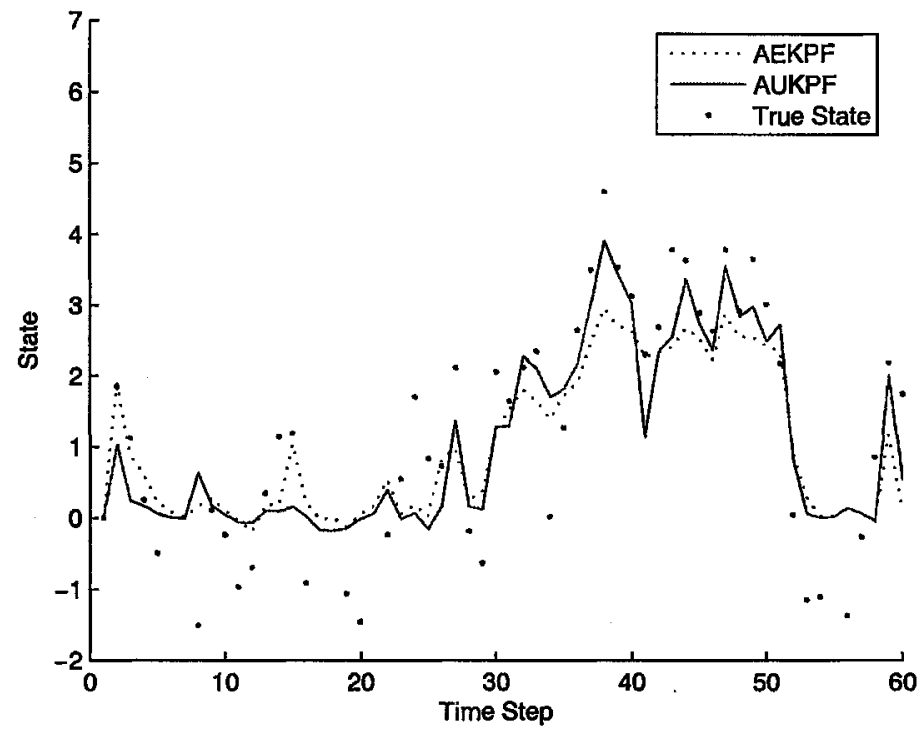

Figure 5.4: AEKPF and AUKPF sample runs with the stochastic volatility model. (RMSE: $\mathrm{AEKPF}=1.14, \mathrm{AUKPF}=1.09$, Worst Error: $\mathrm{AEKPK}=2.82, \mathrm{AUKPF}=2.79$.) 


\subsection{D Target Tracking With Range and Bearing Measurements}

\subsubsection{Introduction}

This section explores the use of the particle filtering methods to track a target on a 2D surface using range and bearing measurement. The target moves at a constant velocity corrupted slightly by noise. Meanwhile, the measurements are received by a manoeuvring observer. Examples of such a scenario arise in many areas of modern warfare. For instance, tracking submarines, aircraft, or missiles $[4,37,56,63]$. An illustration of such an example is shown in Figure 5.5.

Although the target is moving at a constant velocity, the manoeuvring of the observer is nonlinear as are the measurement relations. Nonlinearities in the system dynamics make the tracking problem difficult. Additional complexity in tracking also results from the fact that the target is free to move on a $2 \mathrm{D}$ surface. This increases the dimension of the state vector that is being estimated.

\subsubsection{Model Description}

The basic idea behind target tracking is to estimate the trajectory of a target (i.e., the position and the velocity) from noisy sensor data. Hence, the target state vector is

$$
\bar{x}^{t}=\left[x^{t} y^{t} \dot{x}^{t} \dot{y}^{t}\right]^{T}
$$

where $(x, y)$ and $(\dot{x}, \dot{y})$ are the position and velocity components of the target state respectively. The sensor data is simulated as being acquired by a moving observer. The moving observer's state vector $x^{\circ}$ is defined in a similar fashion to that of the target. The relative state vector is then the difference between the target and observer 


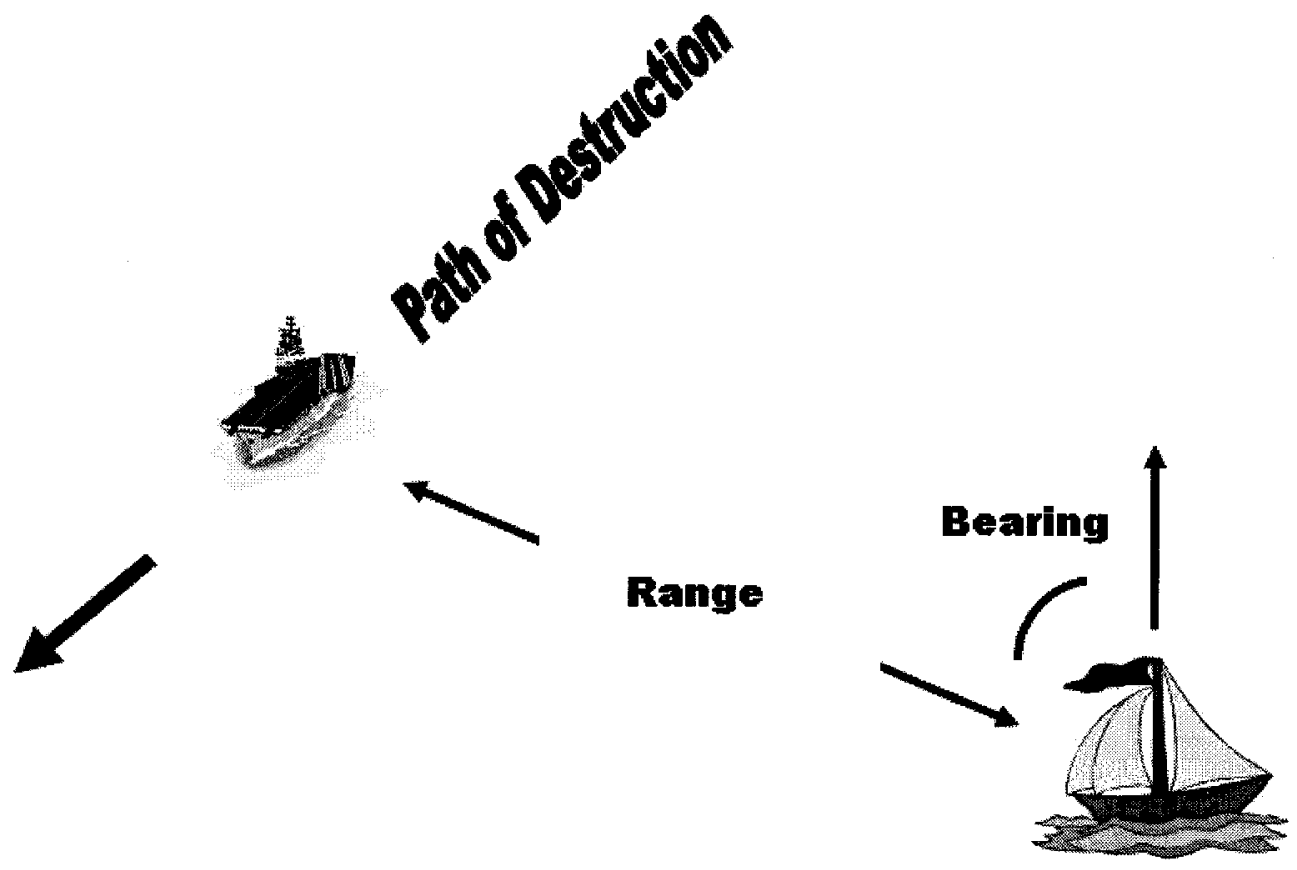

Figure 5.5: Consider a hypothetical application of the tracking problem involving a sailboat and an aircraft carrier. The sailboat is the manoeuvring observer craft in this example. The aircraft carrier is the target. The captain of the sailboat wants to avoid a collision with the aircraft carrier and thus takes bearing and range measurements of the aircraft carrier. The captain uses filtering algorithms to estimate the actual position of the aircraft carrier and assure that his sailboat does not collide with the much larger craft.

state vectors

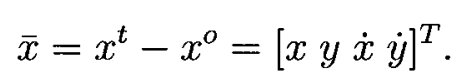

The target dynamics are written mathematically as

$$
\bar{x}_{t+1}=F \bar{x}_{t}+G \bar{v}_{t}-\dot{u}
$$


where $\mathrm{F}$ is the transition matrix

$$
F=\left[\begin{array}{llll}
1 & 0 & T & 0 \\
0 & 1 & 0 & T \\
0 & 0 & 1 & 0 \\
0 & 0 & 0 & 1
\end{array}\right]
$$

$\mathrm{G}$ is an acceleration matrix

$$
G=\left[\begin{array}{cc}
\frac{T^{2}}{2} & 0 \\
0 & \frac{T^{2}}{2} \\
T & 0 \\
0 & T
\end{array}\right]
$$

and $\mathrm{T}$ is the sampling time. The process noise vector represented by $\bar{v}_{t}$ is a $2 \times 1$ vector with mean zero and variance $Q . Q$ is chosen as $Q=\sigma^{2} I$ where $I$ is the $2 \times 2$ identity matrix and $\sigma$ is a process noise parameter selected to match the system. Finally, $\dot{u}$ represents the change in the observers position, $\dot{u}=\left[\begin{array}{lllll}\left(x_{t+1}^{o}-x_{t}^{o}\right) & \left(y_{t+1}^{o}-y_{t}^{o}\right) & 0 & 0\end{array}\right]^{T}$.

The observer's transition matrices, $F_{o}^{(k)}$, are designed to allow for both clockwise and anticlockwise rotation, respectively. These are given by

$$
F_{o}^{(k)}=\left[\begin{array}{cccc}
1 & 0 & \frac{\sin \left(\Omega^{(k)} T\right)}{\Omega^{(k)}} & -\frac{1-\cos \left(\Omega^{(k)} T\right)}{\Omega^{(k)}} \\
0 & 1 & \frac{1-\cos \left(\Omega^{(k)} T\right)}{\Omega^{(k)}} & \frac{\sin \left(\Omega^{(k)} T\right)}{\Omega^{(k)}} \\
0 & 0 & \cos \left(\Omega^{(k)} T\right) & -\sin \left(\Omega^{(k)} T\right) \\
0 & 0 & \sin \left(\Omega^{(k)} T\right) & \cos \left(\Omega^{(k)} T\right)
\end{array}\right], k=1,2
$$

where the turning rates are

$$
\begin{aligned}
& \Omega^{(1)}=\frac{a_{m}}{\sqrt{\left(\dot{x}_{k}+\dot{x}_{k}^{o}\right)^{2}+\left(\dot{y}_{k}+\dot{y}_{k}^{o}\right)^{2}}}, \\
& \Omega^{(2)}=\frac{-a_{m}}{\sqrt{\left(\dot{x}_{k}+\dot{x}_{k}^{o}\right)^{2}+\left(\dot{y}_{k}+\dot{y}_{k}^{o}\right)^{2}}} .
\end{aligned}
$$


The term $a_{m}>0$ is called the manoeuvre acceleration [4]. Clearly, the observer's transitions are nonlinear.

At every time step, $t$, there are two measurements available: range and bearing. The range is the distance from the observer to the target. Meanwhile, the bearing measurement is the angular direction of the target with respect to the observer and a geographical reference. The measurement model used for the simulation is

$$
\begin{aligned}
\bar{z}_{t} & =\bar{w}_{t}+h\left(\bar{x}_{t}\right) \\
& =\bar{w}_{t}+ \begin{cases}\sqrt{\left(y_{t}-y_{t}^{o}\right)^{2}+\left(x_{t}-x_{t}^{o}\right)^{2}} & \text { (range) } \\
\arctan \left(\frac{y_{t}-y_{t}^{o}}{x_{t}-x_{t}^{o}}\right) & \text { (bearing) }\end{cases}
\end{aligned}
$$

where $\bar{w}_{t}$ is a $2 \times 1$ Gaussian noise vector with zero mean and variances of $\sigma_{r}^{2}$ and $\sigma_{b}^{2}$ respectively.

\subsubsection{Jacobian Calculations}

One of the negative aspects of the EKF based filters is that they require the calculation of the state transition and measurement Jacobians

$$
\begin{aligned}
F_{t}^{j} & =\left[\nabla \bar{x}_{t}(F)^{T}\right]^{T} \\
H_{t}^{j} & =\left[\nabla \bar{x}_{t} h^{T}\left(\bar{x}_{t}\right)\right]^{T} .
\end{aligned}
$$

The first Jacobian matrix $F_{t}^{j}$ is simply the trasition matrix $F$ given by Eqn 5.2.2. The Jacobian for the measurement equation is more complicated. It is calculated as follows

$$
H_{t}^{j}=\left[\begin{array}{llll}
\frac{\partial h_{1}}{\partial x_{t}} & \frac{\partial h_{1}}{\partial y_{t}} & \frac{\partial h_{1}}{\partial \dot{x}_{t}} & \frac{\partial h_{1}}{\partial \dot{y}_{t}} \\
\frac{\partial h_{2}}{\partial x_{t}} & \frac{\partial h_{2}}{\partial y_{t}} & \frac{\partial h_{2}}{\partial \dot{x}_{t}} & \frac{\partial h_{2}}{\partial \dot{y}_{t}}
\end{array}\right]
$$


where

$$
\begin{array}{clll}
\frac{\partial h_{1}}{\partial x_{t}}=\frac{x}{\sqrt{x^{2}+y^{2}}} & \frac{\partial h_{1}}{\partial y_{t}}=\frac{y}{\sqrt{x^{2}+y^{2}}} & \frac{\partial h_{1}}{\partial \dot{x}_{t}}=0 & \frac{\partial h_{1}}{\partial \dot{y}_{t}}=0 \\
\frac{\partial h_{2}}{\partial x_{t}}=\frac{-y}{x^{2}+y^{2}} & \frac{\partial h_{2}}{\partial x_{t}}=\frac{x}{x^{2}+y^{2}} & \frac{\partial h_{2}}{\partial \dot{x}_{t}}=0 & \frac{\partial h_{2}}{\partial \dot{y}_{t}}=0 .
\end{array}
$$

The UKF based filters have the advantage that they do not require any Jacobian calculations. Although the calculations required in this example are relatively simple, more complicated systems may have very computationally intensive Jacobian calculations.

\subsubsection{Initialization}

For the simulation the target was initially positioned 400 units north of the observer. The target had an initial velocity of 3 units/time step east and 4 units/time step south. Hence, the initial state vector of the target is

$$
\bar{x}^{0}=\left[\begin{array}{llll}
0 & 4003-4
\end{array}\right]^{T} \text {. }
$$

The observer was initialized with velocities 1 unit/time step east and 1 unit/time step north. The observer's initial state vector is

$$
\bar{x}_{o}^{0}=\left[\begin{array}{llll}
0 & 0 & 1 & 1
\end{array}\right]^{T}
$$

Furthermore, the observer's manoeuvre acceleration was set to 0.4 units/(time step) ${ }^{2}$. The simulation was run with the time step equal to 1 . 
The $Q$ and $R$ values for the filters were set as per follows

$$
\begin{gathered}
Q=\left[\begin{array}{cc}
0.01 & 0 \\
0 & 0.01
\end{array}\right] \\
R=\left[\begin{array}{cc}
100 & 0 \\
0 & 10
\end{array}\right] .
\end{gathered}
$$

It was assumed that the observer has some initial knowledge of the target's speed and course. This information is as listed

- initial range $r \sim N\left(\bar{r}, \sigma_{r}^{2}\right)=N\left(y_{1}, 0.5\right)$

- initial target speed $s \sim N\left(\bar{s}, \sigma_{s}^{2}\right)=N(5,0.1)$

- initial course $c \sim N\left(\bar{c}, \sigma_{c}^{2}\right)=N\left(-\frac{\pi}{4}, 0.001\right)$.

From this information the initial covariance matrix is constructed as

$$
P=\left[\begin{array}{cccc}
\sigma_{x}^{2} & \sigma_{x y}^{2} & 0 & 0 \\
\sigma_{y x}^{2} & \sigma_{y}^{2} & 0 & 0 \\
0 & 0 & \sigma_{\dot{x}}^{2} & \sigma_{\dot{x} \dot{y}}^{2} \\
0 & 0 & \sigma_{\dot{y} \dot{x}}^{2} & \sigma_{\dot{y}}^{2}
\end{array}\right]
$$


where

$$
\begin{aligned}
\sigma_{x}^{2} & =\bar{r}^{2} \sigma_{\theta}^{2} \cos ^{2} \theta_{1}+\sigma_{r}^{2} \sin ^{2} \theta_{1} \\
\sigma_{y}^{2} & =\bar{r}^{2} \sigma_{\theta}^{2} \sin ^{2} \theta_{1}+\sigma_{r}^{2} \cos ^{2} \theta_{1} \\
\sigma_{x y} & =\left(\sigma_{r}^{2}-\bar{r}^{2} \sigma_{\theta}^{2}\right) \sin \theta_{1} \cos \theta_{1} \\
\sigma_{y x} & =\left(\sigma_{r}^{2}-\bar{r}^{2} \sigma_{\theta}^{2}\right) \sin \theta_{1} \cos \theta_{1} \\
\sigma_{\dot{x}}^{2} & =\bar{s}^{2} \sigma_{c}^{2} \cos ^{2}(\bar{c})+\sigma_{s}^{2} \sin ^{2}(\bar{c}) \\
\sigma_{\dot{y}}^{2} & =\bar{s}^{2} \sigma_{c}^{2} \sin ^{2}(\bar{c})+\sigma_{s}^{2} \cos ^{2}(\bar{c}) \\
\sigma_{\dot{x} \dot{y}} & =\left(\sigma_{s}^{2}-\bar{s}^{2} \sigma_{c}^{2}\right) \sin (\bar{c}) \cos (\bar{c}) \\
\sigma_{\dot{y} \dot{x}} & =\left(\sigma_{s}^{2}-\bar{s}^{2} \sigma_{c}^{2}\right) \sin (\bar{c}) \cos (\bar{c}) .
\end{aligned}
$$

For the simulations $\theta_{1}$ was the first bearing measurement and $\bar{r}$ was the first range measurement. The value $\sigma_{\theta}^{2}$ was set as the the measurement models bearing variance, 0.01 .

\subsubsection{Results}

The results from the 2D tracking simulations are shown in Table 5.2. The simulations were run 100 times, each with a different random seed. The results are shown for both 10 and 100 particles. The RMSE value is the mean of the RMSEs over the 100 trials. The variance is calculated over the 100 RMSE values from the independant simulations. Each trial was run for 100 time steps.

The results for this problem are interesting as the EKF with a mean RMSE of 46.54 outperforms the UKF with a mean RMSE of 71.71. The variance of the UKF results are about half that of the EKF results. The particle filters offered improvements 


\begin{tabular}{|l|cc|cc|}
\hline & \multicolumn{2}{|c|}{ RMSE } & \multicolumn{2}{c|}{ Variance } \\
\hline Particles & 10 & 100 & 10 & 100 \\
\hline EKF & 46.54 & 46.54 & 9763.07 & 9763.07 \\
UKF & 71.71 & 71.71 & 4666.11 & 4666.11 \\
EKPF & 40.93 & 37.27 & 4960.36 & 4015.29 \\
\hline AEKPF & 36.82 & 36.61 & 3844.78 & 4062.67 \\
UKPF & 24.78 & 21.90 & 262.16 & 250.67 \\
AUKPF & 40.35 & 33.90 & 359.92 & 171.47 \\
\hline
\end{tabular}

Table 5.2: RMSE and Variance results using 10 and 100 particles for the tracking problem.

to the EKF and UKF filters. Although the UKF results were worse than the EKF results, the UKF based particle filters still performed better than the EKF based filters. The lowest mean RMSE was that of the UKPF. Second best was the AUKPF. The lowest variance, however, was that of the AUKPF. It was much lower than the EKF based particle filters. The UKPF also had a relatively low variance. Overall, the UKF based particle filters seemed to work the best for this problem. Example trial runs are shown in Figure 5.6-5.7.

\subsection{Chapter Summary}

This chapter presented two practical problems that filtering algorithms can be applied to. The first was the stochastic volatility model used in finance. The results indicated that the AUKPF and UKF filters work the best for this kind of problem, although, their results are only marginally better in terms of RMSE than the other filters with the exception of the SIRPF and ASIRPF filters whose performance was much worse than the other filters. The second problem is a $2 \mathrm{D}$ tracking experiment with range and bearing measurements. This problem is different than the others as it has a state vector as opposed to a scalar. The results showed that the UKPF outperformed the 
other filters with the AUKPF as the second best performer. 


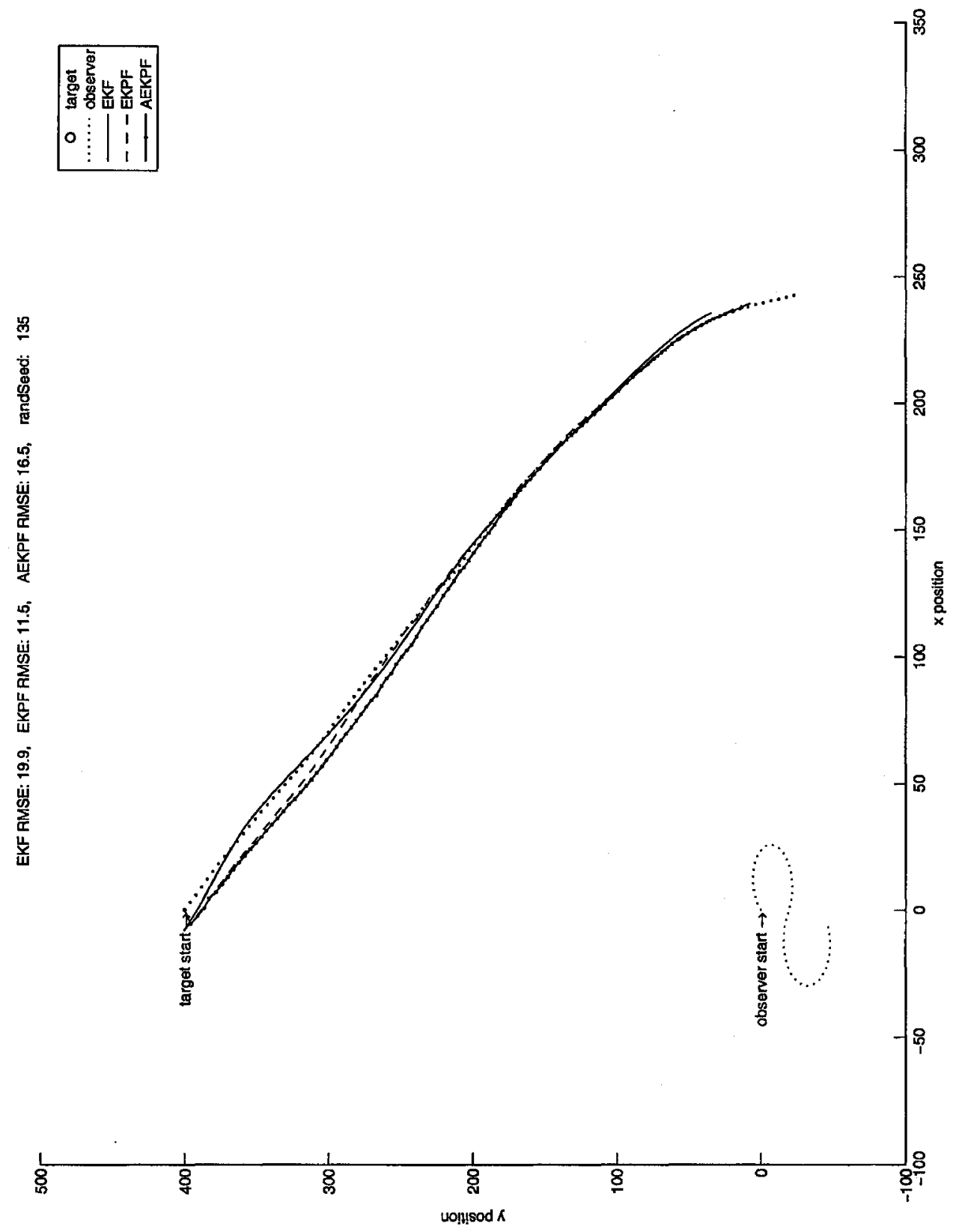

Figure 5.6: EKF based filter results for the 2D tracking problem. 


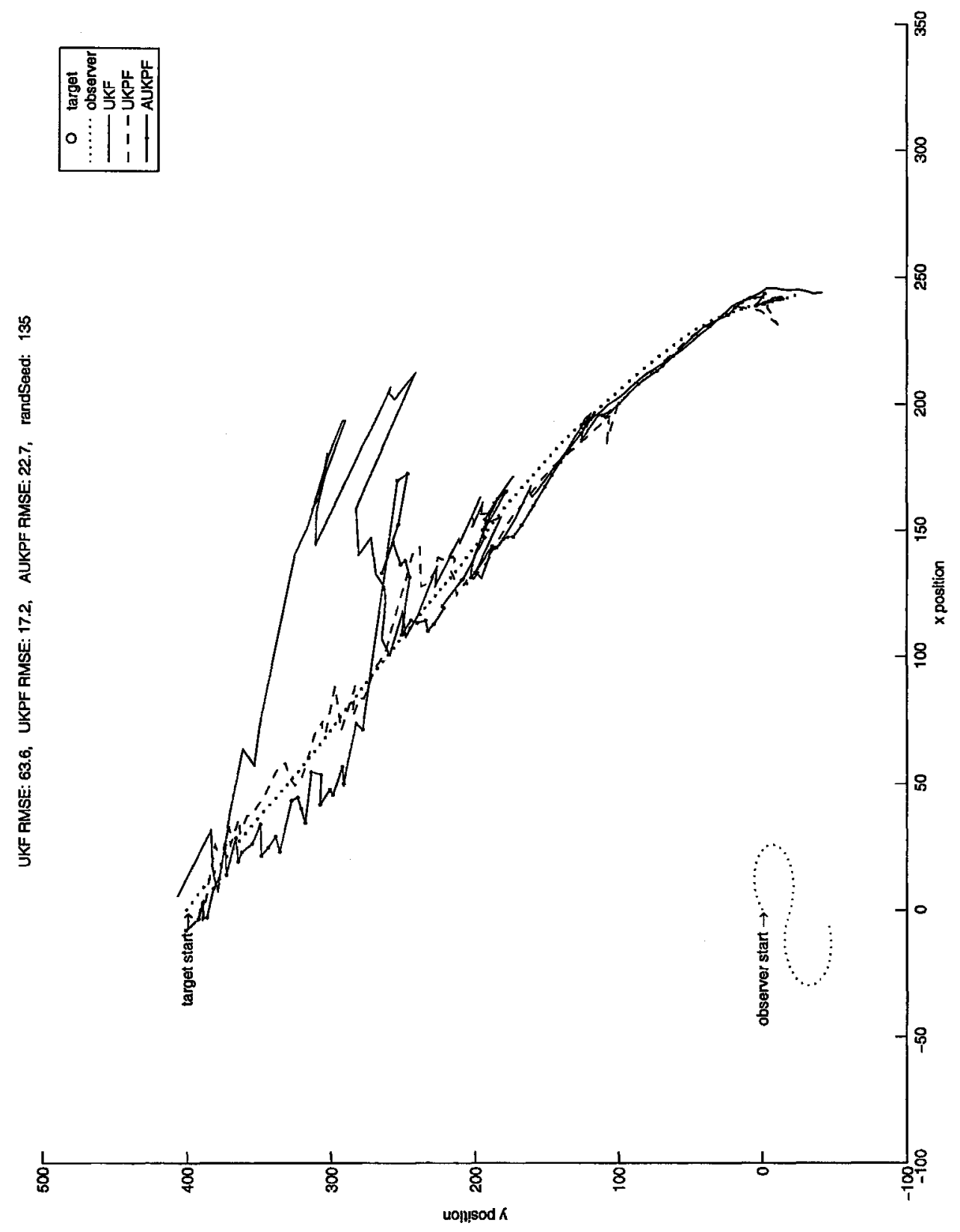

Figure 5.7: UKF based filter results for the 2D tracking problem. 


\section{Chapter 6}

\section{Conclusions and Recomendations}

\subsection{Conclusions}

The Kalman filter has been a popular algorithm since its inception in 1960. In 1979 the nonlinear version, the EKF, was introduced so that the filtering algorithm can be successfully applied to a wider range of problems. More recently in 1997 the UKF was proposed as an alternative to the EKF. The main difference between the two filtering techniques is that the UKF does not approximate the nonlinear system and observation models. Alternatively, the UKF method uses the true system and observation models and instead makes an approximation about the distribution of the state variables. In order to estimate the distribution of the state variables the UKF uses what is known as the unscented transform. A set of deterministically selected sample points are propagated through the true nonlinear system. These points are used to represent the posterior mean and covariance. This can be seen as an advantage over the EKF as the EKF requires the Jacobians of the system to be calculated. Also, no linear approximation is necessary; hence, the UKF can better reflect the true nonlinear system. 
From the results in Chapter 4 it is clear that the UKF outperforms the EKF in terms of RMSE for the scalar cases presented. This is especially evident in the Cauchy noise simulations where the EKFs RMSE results are an order of magnitude greater than the UKFs. For the stochastic volatility simulation the two filters produced similar results. Surprisingly, however, for the $2 \mathrm{D}$ tracking simulations the EKF proved to have a lower RMSE than the UKF over the 100 trial runs.

The cheap computer power abundant in the late $20^{\text {th }}$ century has lead many researchers to the Monte Carlo method. The MC method employs large quantities of random numbers in order to make numerical approximations. For instance, integration can be carried out via the MC method as shown in Chapter 2. In terms of nonlinear filtering, the MC method is used in algorithms known as particle filters. The seminal particle filter is known as the SIRPF or the bootstrap particle filter. It was first proposed in 1993. It is a basic particle filtering method that uses the prior distribution to propagate and weight particles. Since the introduction of the bootstrap particle filter many alternative algorithms have been introduced. For instance, the ASIRPF was introduced to be more robust to outliers than the SIRPF. Like the SIRPF the ASIRPF uses the prior density to propagate and weight particles, however, it does so in a slightly more complicated manner that involves an auxiliary variable in conjunction with the prior density.

The results of the scalar Gaussian noise simulations show that the SIRPF and the ASIRPF perform in a similar manner. The SIRPF had slightly lower RMSE results at all the varying numbers of particles; however, the ASIRPF had slightly lower variance results. It took about 1000 particles for the SIRPF and the ASIRPF filters to work at a similar performance level compared to the other filters in terms of RMSE. At greater noise variance the ASIRPF proved to somewhat outperform the SIRPF. Both the ASIRPF and the SIRPF had similar results for the scalar simulations involving 
Cauchy and Gamma noise terms. For the stochastic volatility simulation the ASIRPF outperformed the SIRPF in terms of RMSE at both 10 and 100 particles, nevertheless, the performance of both the SIRPF and the ASIRPF was much worse than the EKF and UKF for this model.

As an alternative to the prior distribution other proposal densities have been used and documented. Two of such filters that fall into this category are the recent EKPF and the UKPF. These particle filters use the EKF and the UKF to propagate and weight particles instead of the prior. In this case the algorithms become more complicated as the simplifications offered by using the prior distribution are no longer available, however, by using the EKF and UKF the most recently available data is used in the particle calculations suggesting that better filter performance may be achieved.

The performance of the UKPF and the EKPF was much better than that of the EKF, UKF, SIRPF, and the ASIRPF in terms of RMSE for the scalar Gaussian simulations. When the simulation results were split into three sections the UKPF clearly outperformed the other filters including the EKPF. When using higher noise ratios, however, the RMSE results of the UKPF and the EKPF were worse relative to the EKF and the UKF. For the scalar model with Gamma noise, the UKPF outperformed the EKPF. In fact, the UKPF had a RMSE over 100 trials that was less than half of that of the EKPF. For this case the EKPF outperformed the EKF, but fell short of the UKF. Again, for the Cauchy noise case, the UKF based filters completely outperformed the EKF based filters. Overall, the UKF performed slightly better in terms of RMSE than the UKPF for this case. The results of the 2D tracking simulations showed that the UKPF and the EKPF clearly outperformed the EKF and UKF in terms of RMSE. The UKPF actually had the lowest RMSE results over 100 simulations of all the filters tested on this particular model. Overall, the UKPF 
outperformed the EKPF. It was especially effective in Gaussian simulations with low noise levels and the 2D tracking simulation.

Using the auxiliary particle filtering framework as a basis two new filters based on the EKF and UKF were proposed in this thesis. The first new method combines the EKPF with the auxiliary framework to produce the AEKPF while the second SMC method combines the UKPF with the auxiliary method to produce the AUKPF. The AEKPF and the AUKPF involve more elaborate calculations than their predecessors; however, they have the potential to exhibit the advantages of both of their respective foundations.

The AUKPF proved to be the best filter for the scalar Gaussian simulations. The AUKPFs RMSE was on average the lowest for the simulations, however, it can be pointed out the AEKPF had the lowest RMSE for the case using 1000 particles. When broken into the three separate cases of nonlinearity the AUKPF was clearly the best performer in terms of RMSE. The AUKPF and the AEKPF had trouble when the measurement noise was set at higher levels. In fact, the UKF and EKF outperformed the AEKPF and AUKPF when the measurement noise was high. For the simulations involving Gamma noise terms, the AUKPF and AEKPF have the lowest RMSE results. Their RMSE results in the Gamma case are half that of the next best filter. The AEKPF displays poor results for the Cauchy noise case. This is expected due to the very poor performance of the EKF in this case. The AUKPF, on the other hand, performed better than all the other filters tested in this case, including the UKF. Again, the results of the stochastic volatility simulations have all the filters are comparable RMSE and variance numbers. As for the 2D tracking case, besides the UKPF RMSE results, the AUKPF and the AEKPF had the next best results. This shows that the AUKPF and AEKPF can be used for filtering problems where there is a state vector as opposed to just a scalar. The AUKPF and AEKPF take 
more calculations, however, and having a larger state space compounds the number of total calculations needed. Hence, in situations where results are needed extremely quickly the AUKPF and AEKPF may not be the best choices.

The most influential variable on a simulation involving a particle filter is the number of particles used. Using more particles in a simulation requires more calculations to be performed. Hence, using more particles requires more computation time. Consequently, using more particles allows for a more complete exploration of the state space and thus increases potential for better filter performance. The simulations showed that using as few as 10 particles, the particle filtering algorithms can produce results that are comparable to the EKF. Usually, though, using 100 particles increases the performance of the particle filters.

\subsection{Contributions}

This thesis begins introducing the Monte Carlo method, the main idea used in particle filters. This concept was illustrated with Buffon's needle example. A numerical example was also used to show the Monte Carlo Integration method. Next, Gausss early work on linear regression is reviewed and used as a basis on which more modern filtering techniques are built. These filters include the Kalman filter, EKF, and the UKF.

Similar to the Kalman filter that took advantage of the computational power available during its day, the particle filter is too making the most of the currently available machines. The state of the art of particle filtering methods is reviewed including outlines and explanation of the generic and the auxiliary particle filter frameworks, choice of proposal densities, degeneracy, and re-sampling.

Two new particle filters are forged from the auxiliary particle framework using the 
EKF and UKF as proposal densities. Outlines of these filters are given. Additionally, these filters are compared to their predecessors.

The major contribution of this thesis is the collection of results comparing all the aforementioned filters. A series of simulations was conducted with different parameters and noise models to determine the strengths and weaknesses of the various filters. The main metric used to determine filter performance was RMSE. The first series of tests was run with a Gaussian noise term in the transition model. The number of particles used per simulation was varied; also the variance of the noise terms was also changed. The results of observation models with different degrees of nonlinearity were also presented. The second set of simulations was run on a transition model with a Gamma noise term. The performance of the filters with various numbers of particles was examined. A third series of simulations was run on a transition model with Cauchy noise added to it. The effects of the large tails associated with the Cauchy noise distribution are observed. Additionally, the filters were applied to two more practical problems. The first was the stochastic volatility model commonly used in financial research. The second was a more traditional tracking application whereby the filters were used to track a target from a manoeuvring observer. An example of this situation would be two ships at sea using radar measurements. Unlike the previous experiments, this problem involved a state vector as opposed to the scalar case.

\subsection{Recommendations}

The following are a list of recommendations to further the work started in this thesis.

- It was interesting to observe how the filters worked on first one dimensional problems, and then on two dimensional problems. In the future a three dimen- 
sional system model could be tested.

- The major difference in problems of different dimensions is the size of the state space. The filters should be applied to larger state spaces to examine the effects of accuracy and computational time.

- A fascinating problem that researchers are currently applying particle filters to is the bearings only tracking problem. This is a tracking problem that is not always completely observable. The observer must manoeuvre in order to track the target. The newly proposed AUKPF and AEKPF filters could be applied to this problem.

- Another twist on the $2 \mathrm{D}$ tracking problem studied in this thesis is the tracking problem with complex target manoeuvring. In this case the problem may have elements of extreme nonlinearity and may also be non-stationary. Probabilistic jumps may occur in the targets tracks. This would add another element of difficulty to the problem.

- Stochastic control applications could be explored. For instance, the missile guidance problem where the particle filters are used to estimate the state and then the information is used in conjunction with control algorithms in order to move the observer closer to the target.

- A more general problem yet to be solved is that of optimizing all parameters associated with the particle filters. For instance, the UKF based filters have alpha, beta, gamma, R, and Q that have to be set. Even just a set of rules to help determine these values would be useful.

- Similarly to the aforementioned point, determining the number of particles is always still a problem. It is a trade off as more particles may lead to more 
accuracy, however, they require more computational time. Being able to link statistically the number of particles used to a particular performance level could be valuable tool. 


\section{References}

[1] V.C. Aitken. Motion and Structure Estimation in Noisy Monocular Image Sequences. Carleton Univeristy, M.Eng. thesis, Ottawa, ON, 1991.

[2] B. D. O. Anderson and J. B. Moore. Optimal Filtering. Prentice-Hall, Englewood Cliffs, NJ, 1979.

[3] S. Arulampalam, S. Maskell, N. Gordon, and T. Clapp. A tutorial on particle filters for on-line non-linear/non-gaussian bayesian tracking. IEEE Transactions on Signal Processing, 50(2):174-188, February 2002.

[4] S. Arulampalam, B. Ristic, N. Gordon, and T. Mansell. Bearings-only tracking of manoeuvring targets using particle filters. EURASIP Journal on Applied Signal Processing, 15:2351-2365, 2004.

[5] K. Astrom and B. Wittenmark. Adaptive Control. Addison Wesley, 1995.

[6] J. Banks, J. S. Carson II, B. L. Nelson, and D. M. Nicol. Discrete Event System Simulation. Prentice Hall, 2001.

[7] A. Blake, B. Bascle, M. Isard, and J. MacCormick. Statistical models of visual shape and motion. Trans. R. Soc. Lond. A, vol. 356:1283-1302, 1998.

[8] P. Brockwell and R. Davis. Time Series: Theory and Methods. Springer-Verlag, New York, 1991. 
[9] Roberto Casarin. Bayesian monte carlo filtering for stochastic volatility models.

[10] S. Clark. Autonomous land vehicles navigation using millimetre wave radar. $P h D$ Thesis, University of Sidney, 1999.

[11] F. Daum. Nonlinear filters: Beyond the kalman filter. IEEE $A$ and E Systems Magazine, Vol. 20, No. 8, 2005.

[12] N. de Freitas. Rao-blackwellised particle filtering for fault diagnosis. In IEEE Aerospace Conference, 2001.

[13] N. de Freitas. Bayesian methods for neural networks. PhD. Dissertation, University of Cambridge, 1999.

[14] Randal Douc, Olivier Cappe, and Eric Moulines. Comparison of resampling schemes for particle filtering. Proceedings of the 4th International Symposium on Image and Signal Processing and Analysis., 2005:64-69.

[15] A. Doucet, N. de Freitas, and N. Gordon. Sequential Monte Carlo Methods in Practise. Springer-Verlag, New York, 2001.

[16] A. Doucet, N. Gordon, and V. Krishnamurthy. Particle filters for state estimation of jump markov linear systems, 1999.

[17] N. Drakos. Introduction to Monte Carlo Methods. Computational Science Education Project, Computer Based Learning Unit, University of Leeds, 1994.

[18] R. Eckhardt. Stan ulam, john von neumann, and the monte carlo method. Los Alamos Science, Special Issue:131-133, 1987.

[19] A. W. F. Edwards. Three early papers on efficient parametric estimation. Statistical Science, Vol. 12, No. 1 (Feb., 1997):35-38. 
[20] P. Fearnhead. Using random quasi-monte-carlo within particle filters, with application to financial time series. Journal of Computational and Graphical Statistics, 14:751-769, 2005.

[21] R.A. Fisher. On an absolute criterion for fitting frequency curves. Messenger of Mathematics, 41:155-160, 1912.

[22] Dieter Fox, Sebastian Thrun, Wolfram Burgard, and Frank Dellaert. Particle filters for mobile robot localization. In Arnaud Doucet, Nando de Freitas, and Neil Gordon, editors, Sequential Monte Carlo Methods in Practice, New York, 2001. Springer.

[23] P. Galko. Stochastic Processes. University of Ottawa, Faculty of Engineering, ELG 5119 course notes, Fall 2005.

[24] K. F. Gauss. Theoria motvs corporvm coelestivm in sectionibvs conicis solem ambientivm. Hambvrgi, Svmtibvs F. Perthes et I. H. Besser, 1809., 1809.

[25] A. Gelb (Ed.). Applied optimal estimation. MIT Press, Cambridge, 1974.

[26] N.J. Gordon, Salmond D.J., and A.F.M. Smith. Novel approach to nonlinear/non-gaussian bayesian state estimation. Proc. Inst. Elect. Eng. F, 140, no. 2,:107-113, 1993.

[27] J.M. Hammersley and D.C. Handscomb. Monte Carlo Methods. Fletcher and Son Ltd., Norwich, Great Britain, first edition, 1964.

[28] J.E. Handschin. Monte carlo techniques for prediction and filtering of non-linear stochastic processes. Automatica, Vol. 6:555-563, Pergamon Press, 1970.

[29] J. Hull and A. White. The pricing of options on assets with stochastic volatilities. The journal of finance, Vol. XLII, No. 2:281-300, June 1987. 
[30] A. H. Jazwinski. Stochastic Processes and Filtering Theory. Academic Press, New York, 1970.

[31] S. Julier. The scaled unscented transformation. In preparation, 1999.

[32] S. Julier and J. Uhlmann. A new extension of the kalman filter to nonlinear systems. In Int. Symp. Aerospace/Defense Sensing, Simul. and Controls, Orlando, FL, 1997.

[33] R.E. Kalman. A new approach to linear filtering and prediction problems. Transactions of the ASME-Journal of Basic Engineering, 82(Series D):35-45, 1960.

[34] R.E. Kalman and R.S. Bucy. New results in linear filtering and prediction theory. Journal of Basic Engineering, Vol. 83 D:95-108, 1961.

[35] I. Karatzas and S. Shreve. Brownian Motion and Stochastic Calculus. Springer Science+Business Media inc., New York, 1998.

[36] R. Karlsson and N. Bergman. Auxiliary particle filters for tracking a maneuvering target. In IEEE Conference on Decision and Control, Sydney, Australia, 2000.

[37] R. Karlsson and F. Gustafsson. Range estimation using angle-only target tracking with particle filters. Proceedings of the American Control Conference, pages 3743-2748, June 2001.

[38] G. Kitagawa. Monte carlo filter and smoother for non-gaussian nonlinear state space models. J. Comput. Graph. Statist., 5:1-25, 1996.

[39] M. Klaas, J.F.G. De Freitas, and A. Doucet. Towards practical $n^{2}$ monte carlo: The marginal particle filter. Proc. UAI, 2005. 
[40] Daphne Koller and Uri Lerner. Sequential Monte Carlo Methods in Practice, chapter Sampling in Factored Dynamic Systems, pages 445-464. Springer, 2001.

[41] A.N. Kolmogorov. Interpolation and extrapolation of stationary random sequences. Isvestiia Akademii Nauk SSSR, Seriia Matematicheskiia 5. (Translation, 1946, Memo RM-3090-PR, Rand Corp., Santa Monica, CA), pages 3-14, 1941.

[42] A. Kong, J. S. Liu, and W. H. Wong. Sequential imputations and bayesian missing data problems. Journal of the American Statistical Association 89, pages 278-288, 1994.

[43] J. H. Kotecha and P. M. Djuric. Gaussian sum particle filtering. IEEE Transactions on Signal Processing 51, pages 2602-2612, 2003.

[44] Chong K.S. and Kleeman L. Mobile robot map building from an advanced sonar array and accurate odometry. Technical Report MECSE-1996-10, Melbourne, Monash University. Department of Electrical and Computer Systems Engineering. 1996.

[45] K. Lee and S. Koopman. Estimating stochastic volatility models: a comparison of two importance samplers. studies in nonlinear and dynamics and econometrics, Vol. 8, Issue 2, 2004.

[46] M. Legendre, A. Nouvelles Methodes pour la determination des Orbites des Cometes. Courcier, 1806; VIII, 55 p. ; in 4.; DCCC. f. 10, Paris, 1806.

[47] D. H. Lehmer. Mathematical methods in large-scale computing units. In Proc. 2nd Sympos. on Large-Scale Digital Computing Machinery, pages 1141- 146, Harvard University Press, 1951. 
[48] J. S. Liu and R. Chen. Sequential monte carlo methods for dynamic systems. Journal of the American Statistical Association 93, pages 1032-1044, 1998.

[49] A.D. Marrs. Sequential Monte Carlo Methods in Practice, chapter In-Situ Ellipsometry Solutions Using Sequential Monte Carlo, pages 465-477. Springer, 2001.

[50] G. Marsaglia and W. Tsang. The ziggurat method for generating random variables. Journal of Statistical Software, 5(8), 2000.

[51] G. Marsaglia and A. Zaman. A new class of random number generators. Annals of Applied Probability, 3:462-480, 1991.

[52] S. McGinnity and G. Irwin. Sequential Monte Carlo Methods in Practice, chapter Maneuvering target tracking using a multiple-model bootstrap filter, pages 479496. Springer, 2001.

[53] N. Metropolis and S. Ulam. The monte carlo method. Journal of the American Statistical Association, 44 (247):335-341, 1949.

[54] T. Mikosch. Elementary Stochastic Calculas with Finance in View. World Scientific Publishing Co. Pte. Ltd., Singapore, 1998.

[55] C. Moler. Numerical Computing with MATLAB. SIAM, 2004.

[56] S. Nardone, A. Lindgren, and K. Gong. Fundamental properties and performance of conventional bearings-only target motion analysis. IEEE transactions on automatic control, 29 no. 9, Sept, 1984.

[57] A. Papoulis. Probability, Random Variables and Stochastic Processes. McGrawHill, Inc., New York, 1984. 
[58] M. Pitt and N. Shephard. Filtering via simulation: auxiliary particle filters. Journal of the American Statistical Association, 94 no. 446, 1999.

[59] D.G.S. Pollock. A course of Econometrics, Chapter 7: Recursive Estimation and the Kalman Filter. Queen Mary University, London, England, 2001.

[60] S.M. Ross. Introduction to Probability Models. Academic Press, Inc., San Diego, California, seventh edition, 2000.

[61] D. Salmond and N. Gordon. Sequential Monte Carlo Methods in Practice, chapter Particles and mixtures for tracking and guidance, pages 517-528. Springer, 2001.

[62] S. Shreve. Stochastic Calculus for Finance II, Continuous-Time Models. Springer-Verlag, New York, 2004.

[63] T.L. Song. Observability of target tracking with bearings-only measurements. IEEE transactions on aerospace and electronic systems, 32:4, Oct. 1996.

[64] H. W. Sorenson. Least-squares estimation: from gauss to kalman. IEEE Spectrum., 7:63-68, July 1970.

[65] A. Srivastava, A.D. Lanterman, U. Grenander, M. Loizeaux, and M.I. Miller. Sequential Monte Carlo Methods in Practice, chapter Monte-Carlo Techniques for Automated Target Recognition, pages 533-577. Springer, 2001.

[66] Rudolph van der Merwe, Nando de Freitas, Arnaud Doucet, and Eric Wan. The unscented particel filter. In Advances in Neural Information Processing Systems 13, Nov 2001.

[67] E. Wan and R. van der Merwe. The Unscented Kalman Filter for Nonlinear Estimation. Proc. of IEEE Symposium 2000 (AS-SPCC), Lake Louise, Alberta, Canada, Oct. 2000., 2000. 
[68] G. Welch and G. Bishop. An introduction to the kalman filter. ACM SIGGRAPH 2001, Course 8, Los Angles, California, August 2001.

[69] N. Wiener. Extrapolation, Interpolation and Smoothing of Stationary Time Series with Engineering Applications. The MIT Press, Cambridge (Mass.), 1949.

[70] Wikipedia. Gaussmarkov theorem - wikipedia, the free encyclopedia, 2005. [Online; accessed 31-January-2006].

[71] Wikipedia. Random walk — wikipedia, the free encyclopedia, 2006. [Online; accessed 29-January-2006]. 


\section{Appendix A}

\section{Basic Concepts of Stochastic}

\section{Processes}

\section{A.1 Introduction to Random Variables}

This section is motivated by the appendix attached to R.E. Kalman's seminal paper [33] from 1960 on the Kalman filter. Basic mathematical tools and notation that are used throughout the thesis are introduced for the convenience of the reader. This is a relatively short review for the elaborate topic known as stochastic processes. For more information, some good sources are $[30,57,60,23]$.

\section{A.1.1 Probability Space}

Before introducing random variables we must discuss what is known as probability space. Probability space is denoted $(\mathcal{S}, \tilde{\mathcal{F}}, \mathcal{P})$ where $\mathcal{S}$ is the sample space, $\tilde{\mathcal{F}}$ is the event space, and $\mathcal{P}$ is the probability measure. To understand what each of these terms means exactly, let us consider the mathematical language used to examine the outcome of a random experiment; for instance, the roll of a fair die. The sample 
space $\mathcal{S}$ of an experiment is said to be the set of all possible outcomes. In the case of the die experiment, the sample space would be the set of all numbers found on the sides of the die, $\{1,2,3,4,5,6\}$. Subsets of the sample space are known as events. For instance, rolling a $\{2\}$ is an event of the die experiment. All the relevant subsets of a random experiment make up the event space, $\tilde{\mathcal{F}}$. The collection of subsets that make up an event space must be a $\sigma$-algebra, i.e., $\tilde{\mathcal{F}}$ is closed under the complementation and countable union operations. Finally, the probability that an event takes place is a value assigned to an event by the probabilizty measure $\mathcal{P}$. This is essentially a mapping from the event space to the real line with certain restrictions and properties. In the case of the die experiment, each event is mapped to a probability of $1 / 6$. The probability space gives a complete description of the random model of the experiment in question.

There are three axioms that make up the properties of the probability measure. As presented in $[23,17]$, they are:
I. $\mathcal{P}(\mathcal{A}) \geq 0$ for any event $\mathcal{A} \in \tilde{\mathcal{F}}$,
(non-negativity)
II. $\mathcal{P}(\mathcal{S})=1$, (finiteness)

III. If $\mathcal{A}_{1}, \mathcal{A}_{2}, \mathcal{A}_{3}, \ldots$ is a countably infinite sequence ( $\sigma$-additivity) These of pairwise disjoint events, then $\mathcal{P}\left(\bigcup_{i=1}^{\infty} \mathcal{A}_{i}\right)=\sum_{i=1}^{\infty} \mathcal{P}\left(\mathcal{A}_{i}\right)$.

properties can be used to derive all the basic relations in probability theory.

\section{A.1.2 Defining a Random Variable}

A random variable is a rule that assigns a value to every outcome of an experiment or chance event. Random variables can take on any mathematical entity, for instance, real or complex numbers. The random variables used throughout this thesis are real random variables. Referring back to the notion of probability spaces, we can 
rigorously define a real random variable [23]:

A real random variable $X$ over a probability space $(\mathcal{S}, \tilde{\mathcal{F}}, \mathcal{P})$ is a function mapping $\mathcal{S}$ to $\mathbb{R}$ so that the inverse image of any interval $(-\infty, y]$ (for all $y \in \mathbb{R})$ is an event, i.e., for all $y \in \mathbb{R}$,

$$
\{s \mid X(s) \leq y, s \in \mathcal{S}\} \in \tilde{\mathcal{F}}
$$

It is also insightful to note that the sums, products, and functions of random variables are also random variables.

A random variable $X$ can be completely defined in a probabilistic sense by stating the probability that $X$ is less than or equal to some real constant $\mathrm{x}$. This is known as the probability distribution function or cumulative distribution function (cdf) of the random variable $X$. It is written

$$
F_{X}(x)=\mathcal{P}(X \leq x)
$$

The probability density function (pdf) $p_{X}(x)$ is the derivative of $F_{X}(x)$ with respect to $\mathrm{x}$,

$$
p_{X}(x)=\frac{\partial F_{X}(x)}{\partial x}
$$

The pdf has two properties. First is that it is non-negative:

$$
f_{X}(x) \geq 0
$$

The second property of the pdf is that the area under the curve $f_{X}(x)$ is unity:

$$
\int_{-\infty}^{+\infty} f_{X}(x) d x=1
$$


Written in a more convenient fashion, the pdf/cdf relation is

$$
F_{X}(a)=\int_{-\infty}^{a} p_{X}(x) d x
$$

The cdf and pdf are powerful tools from probability theory that can be used to solve various mathematical problems.

Previously, we defined the events $X \leq x$ and $Y \leq y$ as having probabilities given by $F_{X}(x)$ and $F_{Y}(y)$ respectively. Now we examine the chance of both events happening. To do this we require that each of the random variables involved is defined on the same probability space. Furthermore, we define the joint distribution function

$$
F_{X Y}(x, y)=\mathcal{P}(X \leq x, Y \leq y)
$$

The joint distribution function shown above is for two random variables, however, it generalizes to any number of random variables. We can also define the joint density function

$$
f_{X Y}(x, y)=\frac{\partial^{2}}{\partial x \partial y} F_{X Y}(x, y)
$$

The joint density function can also be generalized for any number of random variables. A nice feature of the joint density function is that random variables can be "integrated out" in order to achieve marginal density functions. For instance, in the case of the random variables $X$ and $Y$, we can attain the marginal density of $X$ by integrating over $\mathrm{Y}$

$$
f_{X}(x)=\int_{-\infty}^{\infty} f_{X Y}(x, y) d y
$$

Another term used frequently is independence. Two events $\mathcal{A}=\{X \leq x\}$ and $\mathcal{B}=\{Y \leq y\}$ are said to be independent if their joint probability is equal to the 
product of their marginal probabilities, i.e.,

$$
\mathcal{P}(\mathcal{A B})=\mathcal{P}(\mathcal{A}) \mathcal{P}(\mathcal{B})
$$

Additionally, using the joint density function notation, the two random variables, $\mathrm{X}$ and $Y$ are said to be independent if

$$
F_{X Y}(x, y)=F_{X}(x) F_{Y}(y)
$$

\section{A.1.3 Mathematical Expectation}

The expected value of a random variable (also known as the mean of expectation) is the average value of the random variable using the pdf as a weight. Using the Lebesque-Stieltjes integral the expected value of some random variable $\mathrm{X}$ is

$$
\mathcal{E}[X]=\int_{-\infty}^{\infty} x d F_{X}(x)=\int_{-\infty}^{\infty} x p_{X}(x) d x
$$

The expectation should not be confused with the node which is the value of the random variable corresponding to the largest pdf value, assuming such a value exists.

\section{A.1.4 Moments}

Moments provide a means to measure the distribution of a random variable. The set of moments of a random variable $X$ are defined as

$$
m_{n}=\mathcal{E}\left[X^{n}\right]
$$


where $n \geq 0$ and $m_{n}$ is the $n^{\text {th }}$ moment of $\mathrm{X}$. Clearly the first moment is just the expectation of the random variable. Additionally, the central moment is defined as

$$
\mu_{n}=\mathcal{E}\left[(X-\mathcal{E}[X])^{n}\right]
$$

The second central moment is a very important idea for random variables. It is known as the variance of the random variable. It essentially is the measure of the spread of the values about the mean of the random variable. Commonly the variance of the random variable $\mathrm{X}$ is denoted

$$
\operatorname{var}(X)=\sigma_{X}^{2}=\mathcal{E}\left[(X-\mathcal{E}[X])^{2}\right]
$$

Another term frequently referred to is the standard deviation. It is simply the square root of the variance, $\sigma_{X}$.

\section{A.1.5 Joint Moments}

The Joint moments of two random variables $\mathrm{X}$ and $\mathrm{Y}$ are defined as

$$
m_{i, j}=\mathcal{E}\left[X^{i} Y^{j}\right]=\int_{\infty}^{\infty} \int_{\infty}^{\infty} x^{i} y^{j} f_{X Y}(x, y) d x d y
$$

for $i, j \geq 0$. The order of the moment is $i+j$. The first and second order moments are the most widely used, they are:

$$
m_{0,1}=\mathcal{E}[Y] \quad m_{1,0}=\mathcal{E}[X] \quad m_{1,1}=\mathcal{E}[X Y] . \quad m_{0,2}=\mathcal{E}\left[Y^{2}\right] \quad m_{2,0}=\mathcal{E}\left[X^{2}\right] . \quad \text { The }
$$
moment $m_{1,1}$ is the correlation of $\mathrm{X}$ and $\mathrm{Y}$. It is usually denoted $\operatorname{Cor}(X, Y)$.

Combining the results of the second order moments we can produce the correlation matrix of $\mathrm{X}$ and $\mathrm{Y}$. It has the form 


$$
R_{X Y}=\left(\begin{array}{cc}
m_{2,0} & m_{1,1} \\
m_{1,1} & m_{0,2}
\end{array}\right)=\left(\begin{array}{cc}
\mathcal{E}\left[X^{2}\right] & \mathcal{E}[X Y] \\
\mathcal{E}[X Y] & \mathcal{E}\left[Y^{2}\right]
\end{array}\right)
$$

In a similar fashion, the joint central moments of $\mathrm{X}$ and $\mathrm{Y}$ are defined

$$
\mu_{i, j}=\mathcal{E}\left[(X-\mathcal{E}[X])^{i}(Y-\mathcal{E}[Y])^{j}\right]
$$

with $i, j \geq 0$ and $i+j$ as the order of the central moment. The second order joint central moments are especially important. The central moment $\mu_{1,1}$ has been coined the covariance of $\mathrm{X}$ and $\mathrm{Y}$ and is denoted $\operatorname{Cov}(X, Y)$. Notice the covariance and the correlation of random variable are related

$$
\operatorname{Cov}(X, Y)=\operatorname{Cor}(X, Y)-\mathcal{E}[X] \mathcal{E}[Y]
$$

Using the second order central moments, we can produce the covariance matrix of $\mathrm{X}$ and $\mathrm{Y}$

$\Lambda_{X Y}=\left(\begin{array}{cc}\mu_{2,0} & \mu_{1,1} \\ \mu_{1,1} & \mu_{0,2}\end{array}\right)=\left(\begin{array}{cc}\mathcal{E}\left[(X-\mathcal{E}[X])^{2}\right] & \mathcal{E}[(X-\mathcal{E}[X])(Y-\mathcal{E}[Y])] \\ \mathcal{E}[(X-\mathcal{E}[X])(Y-\mathcal{E}[Y])] & \mathcal{E}\left[(Y-\mathcal{E}[Y])^{2}\right]\end{array}\right)$

\section{A.2 Conditional Probability}

Sometimes it is necessary to apply conditions on events in order to specify a probabilistic experiment. For instance, consider the famous "The Monte Hall Problem" from the "Let's Make a Deal" television game show. During part of the show a contestant guesses which of three large doors hides a cash prize. The host, Monte Hall, then reveals to the contestant who has just chosen a door, that the prize is not behind one of the unselected doors. Usually a goat of some sort is revealed. At this point 

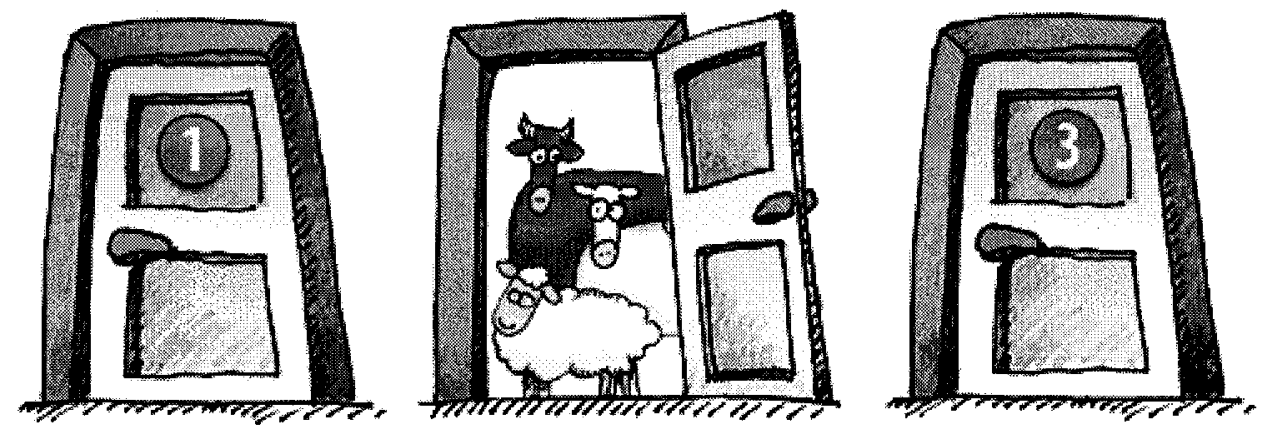

Figure A.1: The Monte Hall Problem (artwork courtesy of cartalk.cars.com).

Hall offers the contestant the opportunity to change their guess to the remaining unopened door.

It is also at this point while sitting in their respective living rooms that many people have postulated if changing doors offers any advantage to the contestant. The naive game show philosopher usually comes to the conclusion that it cannot help the contestant to switch doors. Such a couch creature argues that after the door has been opened there are two doors left and that the prize is equally likely to be behind either one. Thus, there is no benefit to switching doors. Luckily, for the game show contestant, a grad student in a cage somewhere has come up with another slightly more intelligent solution to this problem of the ages. Using conditional probability, it can be shown that changing doors is probably advantageous to the contestant who prefers cash prizes to goats.

To show this we first must define the problem in a mathematical context. Let us name the doors $\mathrm{a}, \mathrm{b}$, and $\mathrm{c}$. Then let there be three events $\mathrm{A}, \mathrm{B}$, and $\mathrm{C}$. $\mathrm{A}$ is the event that the prize is behind door a. B is the event that the prize is behind door $\mathrm{b}$, and finally, $\mathrm{C}$ is the event that the prize is behind door c. We will say that the contestant picks door a and that the host then reveals either $B^{c}$ or $C^{c}$, that is, that 
the prize is not behind door $b$, or that the prize is not behind door c. Again, let us assume that the host shows that there is no prize behind door b. In this case, we want to calculate

$\mathcal{P}\left(C\right.$ given the contestant picks $\mathrm{A}$ and that the host reveals $\left.B^{c}\right)$.

Using the rule of conditional probability, one can reason that this is equivalent to $\frac{\mathcal{P}\left(\mathrm{C} \text { and the contestant picks } \mathrm{A} \text { and the host reveals } B^{c}\right)}{\mathcal{P}\left(\text { the contestant picks } \mathrm{A} \text { and the host reveals } B^{c}\right)}$.

To calculate this expression we must make assumptions about the game. The first assumption is that the host decides randomly which door to reveal if the contestant is correct with their initial choice. This is important because the event that the contestant can pick $\mathrm{A}$ and the host reveals $B^{c}$ can happen in two ways, either the prize is behind door a or door c. In that case,

$\mathcal{P}\left(\right.$ the contestant picks $\mathrm{A}$ and the host reveals $\left.B^{c}\right)=$ $\mathcal{P}$ (A and the contestant picks $\mathrm{A}$ and the host reveals $B^{c}$ ) $+\mathcal{P}\left(\mathrm{C}\right.$ and the contestant picks $\mathrm{A}$ and the host reveals $\left.B^{c}\right)$. 
For calculate the first term we again use the rule of conditional probability to get

$\mathcal{P}\left(\mathrm{A}\right.$ and the contestant picks $\mathrm{A}$ and the host reveals $\left.B^{c}\right)=$ $\mathcal{P}$ (the host reveals $B^{c}$ given $\mathrm{A}$ and the contestant picks $\mathrm{A}$ ) $\cdot \mathcal{P}(\mathrm{A}$ and the contestant picks $\mathrm{A})$

$$
\begin{gathered}
=\frac{1}{2} \cdot \frac{1}{9} \\
=\frac{1}{18} .
\end{gathered}
$$

To calculate the second term we must make another assumption, that is that the hiding of the prize is independent of the contestant's choice of a door. In that case, if the prize is behind door $\mathrm{c}$ and the contestant picks $\mathrm{a}$, this implies that the host reveals that there is no prize behind door $b$. Then

$\mathcal{P}\left(\mathrm{C}\right.$ and the contestant picks $\mathrm{A}$ and the host reveals $\left.B^{c}\right)=$ $\mathcal{P}$ (the contestant picks A and the host reveals $B^{c}$ )

$$
\begin{gathered}
=\frac{1}{3} \cdot \frac{1}{3} \\
=\frac{1}{9} .
\end{gathered}
$$

Putting these numbers together to solve the initial problem we see that the chance of getting the prize if the contestant switches doors is $\frac{\left(\frac{1}{9}\right)}{\left(\frac{1}{9}+\frac{1}{18}\right)}=\frac{2}{3}$. Since the probability of selecting the correct door on the first guess is only $\frac{1}{3}$, the contestant gains an advantage by switching doors. The basic rules of conditional probability are discussed in the next section. They are very useful in the development of the particle filters discussed later in this thesis. 


\section{A.2.1 Basic Relations of Conditional Probability}

To define a conditional probability two events must be considered, for instance events $\mathcal{A}$ and $\mathcal{B}$. It is assumed that $\mathcal{P}(\mathcal{B})>0$. The conditional probability of $\mathcal{A}$ given $\mathcal{B}$ is then

$$
\mathcal{P}(\mathcal{A} \mid \mathcal{B})=\frac{\mathcal{P}(\mathcal{A B})}{\mathcal{P}(\mathcal{B})}
$$

where $\mathcal{P}(\mathcal{A B})$ is the probability of both events $\mathcal{A}$ and $\mathcal{B}$ occurring. Furthermore, let us assume that $\mathcal{A}$ can be partitioned into $\mathcal{A}_{1}, \mathcal{A}_{2}, \mathcal{A}_{3}, \ldots, \mathcal{A}_{n}$ with $\mathcal{P}\left(\mathcal{A}_{i}\right)>0$ for all $i$, and that $\mathcal{A}$ is the complete sample space, $\mathcal{S}$. Using the additivity axiom of probability, the probability of event $\mathcal{B}$ is

$$
\begin{aligned}
\mathcal{P}(\mathcal{B}) & =\mathcal{P}\left(\mathcal{B}\left(\mathcal{A}_{1}, \mathcal{A}_{2}, \mathcal{A}_{3}, \ldots, \mathcal{A}_{n}\right)\right) \\
& =\mathcal{P}\left(\mathcal{B A}_{1}\right)+\mathcal{P}\left(\mathcal{B A}_{2}\right)+\cdots+\mathcal{P}\left(\mathcal{B A}_{n}\right)
\end{aligned}
$$

Using the conditional probability relation defined previously we get the Theorem of Total Probability

$$
\mathcal{P}(\mathcal{B})=\mathcal{P}\left(\mathcal{B} \mid \mathcal{A}_{1}\right) \mathcal{P}\left(\mathcal{A}_{1}\right)+\cdots+\mathcal{P}\left(\mathcal{B} \mid \mathcal{A}_{n}\right) \mathcal{P}\left(\mathcal{A}_{n}\right)
$$

Consider now the conditional probability of one partition of $\mathcal{A}$, say $\mathcal{A}_{i}$. Assuming $\mathcal{P}(\mathcal{B})>0$,

$$
\mathcal{P}\left(\mathcal{A}_{i} \mid \mathcal{B}\right)=\frac{\mathcal{P}\left(\mathcal{A}_{i} \mathcal{B}\right)}{\mathcal{P}(\mathcal{B})}=\frac{\mathcal{P}\left(\mathcal{B} \mid \mathcal{A}_{i}\right) \mathcal{P}\left(\mathcal{A}_{i}\right)}{\mathcal{P}(\mathcal{B})}
$$

By applying the Theorem of Total Probability to the denominator we get

$$
\mathcal{P}\left(\mathcal{A}_{i} \mid \mathcal{B}\right)=\frac{\mathcal{P}\left(\mathcal{B} \mid \mathcal{A}_{i}\right) \mathcal{P}\left(\mathcal{A}_{i}\right)}{\mathcal{P}\left(\mathcal{B} \mid \mathcal{A}_{1}\right) \mathcal{P}\left(\mathcal{A}_{1}\right)+\cdots+\mathcal{P}\left(\mathcal{B} \mid \mathcal{A}_{n}\right) \mathcal{P}\left(\mathcal{A}_{n}\right)}
$$


which is known as Baye's Rule. This is a famous result that is often used to relate conditional probabilities. It is used in this thesis in the work relating to the particle filters.

\section{A.2.2 Conditional Functions}

In this section we introduce the notions of distribution and density functions of random variables conditioned on events with non-zero probability. Consider the cdf of a random variable $\mathrm{X}$ conditioned on event $\mathcal{M}$ with $\mathcal{P}(\mathcal{M})>0$

$$
F_{X}(x \mid \mathcal{M})=\mathcal{P}(X \leq x \mid \mathcal{M})=\frac{\mathcal{P}(\{X \leq x\} \mathcal{M})}{\mathcal{P}(\mathcal{M})}
$$

The pdf is then simply the derivative of the cdf

$$
p_{X}(x \mid \mathcal{M})=\frac{d}{d x} F_{X}(x \mid \mathcal{M})
$$

We can also extend the notion of expectation to cover the conditional case. Again, using the random variable $\mathrm{X}$ conditioned on event $\mathcal{M}$ with $\mathcal{P}(\mathcal{M})>0$

$$
\mathcal{E}[X \mid \mathcal{M}]=\int_{-\infty}^{\infty} x d F_{X}(x \mid \mathcal{M})=\int_{-\infty}^{\infty} x p_{X}(x \mid \mathcal{M}) d x
$$

Furthermore, using the Theorem of Total Probability, the expectation of $\mathrm{X}$ can be expressed

$$
\mathcal{E}[X]=\mathcal{E}\left[X \mid \mathcal{A}_{1}\right] \mathcal{P}\left(\mathcal{A}_{1}\right)+\mathcal{E}\left[X \mid \mathcal{A}_{2}\right] \mathcal{P}\left(\mathcal{A}_{2}\right)+\cdots+\mathcal{E}\left[X \mid \mathcal{A}_{n}\right] \mathcal{P}\left(\mathcal{A}_{n}\right)
$$

where $\mathcal{A}_{1}, \mathcal{A}_{2}, \mathcal{A}_{3}, \ldots, \mathcal{A}_{n}$ is a set of events with non-zero probability that partition $\mathcal{S}$. When dealing with more than one random variable it becomes necessary to work 
with conditional distribution and density functions. Setting the event $\mathcal{M}$ such that $\mathcal{M}=\{Y \leq y\}$ in Eqn A.2.2 we get that

$$
F_{X}(x \mid Y \leq y)=\frac{F_{X Y}(x, y)}{F_{Y}(y)}
$$

assuming, $F_{Y}(y) \neq 0$. With a little work, the corresponding density function becomes

$$
p_{X}(x \mid Y \leq y)=\frac{p_{X Y}(x, y)}{p_{Y}(y)}
$$

assuming, $p_{Y}(y) \neq 0$. Using Eqn A.2.2 it is clear that the following relation holds

$$
p_{X}(x \mid Y \leq y) p_{Y}(y)=p_{Y}(y \mid X \leq x) p_{X}(x) .
$$

\section{A.3 Stochastic Processes}

A random variable is a rule that assigns a number to every outcome of an experiment. Similarly, a stochastic process is a rule that assigns a function to every outcome. Hence, a stochastic process is a collection of random variables indexed by the parameter set $\mathcal{T}$,

$$
\left\{X_{t} \mid t \in \mathcal{T}\right\}=X_{t \in \mathcal{T}}
$$

The set $\mathcal{T}$ is referred to as the parameter space. Meanwhile, the set of values that the random variables may assume for some $t \in \mathcal{T}$ is called the state space $\mathcal{X}$.

A stochastic process, $X_{t}$ has a discrete state space if the corresponding family of random variables are discrete, otherwise, if the random variables are continuous, the process possesses a continuous state space. Similarly, if the parameter $\mathcal{T}$ is discrete, $(\mathcal{T}=\{1,2, \ldots, n\}, \mathcal{T}=\{1,2, \ldots\})$, the stochastic process is a discrete parameter pro- 
cess. If the parameter set $\mathcal{T}$ is continuous, $(\mathcal{T}=[0,1], \mathcal{T}=\{t: t \geq 0\})$, the stochastic process is a continuous parameter process. Furthermore, a continuous state space, discrete parameter process is referred to as a random sequence, while a continuous state space, continuous parameter process is known as a stochastic process.

A realization of the process can occur for each $\mathcal{X}$. If the parameter set $\mathcal{T}$ is discrete, the realization is a sample sequence, conversely, if the parameter set $\mathcal{T}$ is continuous, the realization is a sample function. If both $t$ and $\mathcal{X}$ are fixed, then $X_{t}$ is either a scalar or vector of scalars.

Many physical processes are modelled as stochastic processes...for example discrete: random walk...derive continuous: Brownian motion....used for stock market modelling, physics of a particle suspended in solution

Many physical processes are modelled as stochastic processes. Perhaps the simplest example is what is known as the random walk. An extremely basic version of the random walk is a path that is calculated as follows:

1. A starting point is chosen.

2. The distance between any two points in the path is constant.

3. The direction the path takes from one point to another is random with each direction being equally probable.

After reviewing these rules it is easy to see why the random walk is sometimes referred to as the "drunkard's walk." It could easily be used to model the movements of a drunkard as he stumbles around town after leaving the local neighbourhood pub in the early hours of the morning.

Another common, yet, more complicated stochastic process that has proven to be very important is Brownian motion [8, 35, 54,62]. Brownian motion is named after the botanist Robert Brown. In 1828, Brown observed the irregular movement 


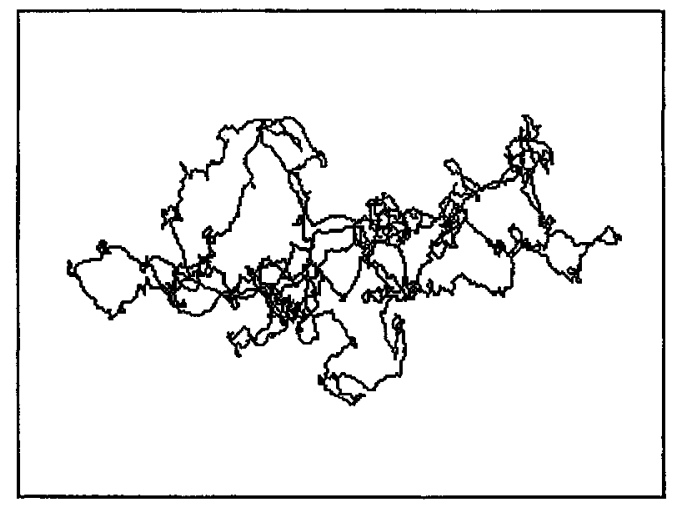

Figure A.2: A sample path of Brownian motion in two dimensions. (Image courtesy of http://classes.yale.edu/fractals/RandFrac/fBm/fBm.html.)

of pollen suspended in water. This random movement results in a diffusion of the pollen in the water.

It was in 1905 that Einstein first postulated that the motion may be caused by the collisions of the particles under consideration with molecules of the surrounding medium. Furthermore, he showed that $p_{X}\left(x, t / x_{0}\right)$ satisfies the partial differential equation

$$
\frac{\partial p}{\partial t}=D \frac{\partial^{2} p}{\partial x^{2}}
$$

where $p_{X}\left(x, t / x_{0}\right)$ is the conditional pdf of $X_{t+t_{0}}$ given that $X_{t_{0}}=x_{0}$. Eqn A.3 is known as the diffusion equation. Heuristically, this can be shown as an extension of a symmetric random walk process. The symmetric random walk of the $\mathrm{x}$-axis only allows the path to either increase or decrease a constant amount along the $\mathrm{x}$-axis. For instance, if the step size is +1 and the path is currently at +3 , the path can either go to +2 or +4 with the next step. Both cases, to +2 and +4 are equally likely. Using the symmetric random walk we define

$$
p_{k}(n+1)=\frac{1}{2} p_{k+1}(n)+\frac{1}{2} p_{k-1}(n)
$$


where $p_{k}(n+1)$ is the probability that the particle is at point $\mathrm{k}$ in stage $\mathrm{n}+1$ of the walk. This implies

$$
\begin{aligned}
p_{k}(n+1)-p_{k}(n) & =\frac{1}{2} p_{k+1}(n)+\frac{1}{2} p_{k-1}(n)-p_{k(n)} \\
& =\frac{1}{2}\left(p_{k+1}(n)-2 p_{k}(n)+p_{k-1}(n)\right)
\end{aligned}
$$

Let $\Delta$ be the time between transitions and $h$ be the length of each step, then

$$
\frac{p_{k h}((n+1) \Delta)-p_{k h}(n \Delta)}{\Delta}=\frac{p_{(k+1) h}(n \Delta)-2 p_{k h}(n \Delta)+p_{(k-1) h}(n \Delta)}{2 \Delta}
$$

Taking the limits as $\Delta=h^{2} \rightarrow 0$ and $n, k \rightarrow \infty$ such that $\rightarrow x$ and $n \Delta \rightarrow t$ we recognize the first term to be the first partial derivative with respect to time while the second term, from the method of finite differences, is an approximation to the second partial derivative with respect to $\mathrm{x}$. Thus, using a symmetric random walk we have shown that $p_{X}\left(x, t / x_{0}\right)$ satisfies the diffusion equation. It is known that the unique solution to the diffusion equation is

$$
p_{X}\left(x, t / x_{0}\right)=\frac{1}{\sqrt{2 \pi t}} e^{-\frac{\left(x-x_{0}\right)^{2}}{2 t}}
$$

which is a form of the Gaussian distribution (see section A.3.2). Brownian motion and its variations have many practical applications in modelling covering a broad range of subjects including option pricing, quantum field theory, and polymer physics $[71]$.

\section{A.3.1 Properties of Stochastic Processes}

In the field of engineering, two of the most important characterizations of a stochastic process are the mean function and the autocorrelation function. Given a stochastic 
process $X_{t}$, the mean function is defined

$$
\eta_{x}(t)=\underset{\mathcal{E}}{\mathcal{E}}\left[X_{t}\right]=\int_{-\infty}^{\infty} x d F_{X}(t ; x)
$$

Meanwhile, given $X_{t}$ and $t_{1}, t_{2} \in \mathcal{T}$, the autocorrelation function is defined

$$
R_{X}\left(t_{1}, t_{2}\right)=\mathcal{E}\left[X_{t_{1}} X_{t_{2}}\right]=\operatorname{cor}\left(X_{t_{1}}, X_{t_{2}}\right)
$$

Using the mean and autocorrelation functions, a third well known characterization can be defined. It is known as the auto-covariance function, denoted

$$
C_{X}\left(X_{t_{1}}, X_{t_{2}}\right)=\operatorname{cov}\left(X_{t_{1}}, X_{t_{2}}\right)=R_{X}\left(X_{t_{1}}, X_{t_{2}}\right)-\eta_{X}\left(t_{1}\right) \eta_{X}\left(t_{2}\right)
$$

A stochastic process is said to be uncorrelated if the auto-covariance is zero, i.e.,

$$
\mathcal{E}\left[X_{t_{1}} X_{t_{2}}\right]=\mathcal{E}\left[X_{t_{1}}\right] \mathcal{E}\left[X_{t_{2}}\right]
$$

Furthermore, if $\mathcal{E}\left[X_{t_{1}} X_{t_{2}}\right]=0$ then the stochastic process is said to be orthogonal.

The statistics of a stochastic process may change with time. A stochastic process is said to be stationary if the statistics do not change with time. A time shifted version of a stationary stochastic process is stochastically equivalent to the original version. This is illustrated in Fig A.3, that was motivated by a similar schematic in [23]. There are certain restrictions governing the mean and autocorrelation functions of a stationary process. The mean function must remain the same with any time shift, and hence, is constant over all $t \in \mathcal{T}$ :

$$
\eta_{X}(t)=\mathcal{E}\left[X_{t}\right]=\mathcal{E}\left[X_{t+\tau}\right]=\eta_{X}(t+\tau)=\eta_{X}
$$




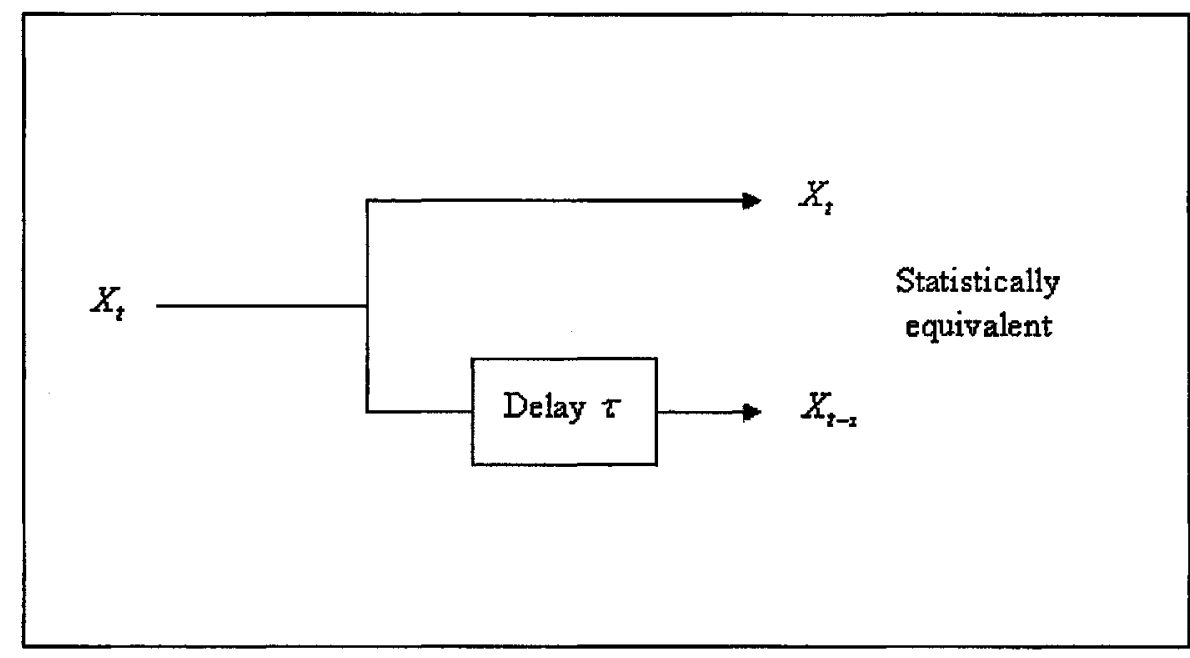

Figure A.3: A graphical interpretation of stationarity.

Similarly, we get

$$
R_{X}\left(t_{1}, t_{2}\right)=\mathcal{E}\left[X_{t_{1}} X_{t_{2}}\right]=\mathcal{E}\left[X_{t_{1}-\tau} X_{t_{2}-\tau}\right]=R_{X}\left(t_{1}+\tau, t_{2}+\tau\right)
$$

for the autocorrelation function when we apply a time shift. Setting the time shift $\tau=-t_{1}$

$$
R_{X}\left(t_{1}, t_{2}\right)=R_{X}\left(0, t_{2}-t_{1}\right)
$$

This is a function of only one argument, thus, we can redefine the autocorrelation function for a stationary process to be

$$
R_{X}(\tau)=\mathcal{E}\left[X_{t} X_{t+\tau}\right]=R_{X}(t, t+\tau)
$$

\section{A.3.2 Gaussian Processes}

As we have seen, the characteristics of random variables are defined by their respective distribution functions. One of the most well known random variables is the Gaussian 
(or Normal) random variable. The Gaussian pdf is defined as

$$
p_{X}(x)=\frac{1}{\sqrt{2 \pi \sigma^{2}}} \exp ^{-\frac{(x-\mu)^{2}}{2 \sigma^{2}}}
$$

where $\mu$ is the mean of $\sigma^{2}$ is the variance. A common shorthand notation for a Gaussian random variable $\mathrm{X}$ is $X N\left(\mu, \sigma^{2}\right)$.

The graph of the Gaussian pdf is the well known bell curve. The Gaussian distribution's importance in probability theory and statistics is made clear by the Central Limit Theorem which is discussed in section A.4.2.

A Gaussian process is a stochastic process $X_{t \in \mathcal{T}}$ such that every finite linear combination of the process is normally distributed. A Gaussian process is uniquely specified by its mean and autocorrelation functions. It is important to note that Gaussian random processes remain Gaussian processes under any linear transformation, however, this is not the case for nonlinear transformations.

It is quite frequent that models of physical or real-world systems come up short when trying to completely encapsulate the dynamics of a system. Unpredictable fluctuations ofter separates the real data from the simulation data. This is known as noise.

In many cases, white noise is used to model the real-world system's noisy behaviour. Mathematically, a white random sequence $\left\{X_{n}, n=1,2,3, \ldots\right\}$ is a Markov sequence whereby all the $X_{i}^{\prime}$ 's are mutually independent, i.e.,

$$
\mathcal{P}\left(X_{i} / X_{j}\right)=\mathcal{P}\left(X_{i}\right) \quad(i>j)
$$

The realization of $X_{j}$ has no information that can help predict $X_{i}$, and thus a white sequence is totally unpredictable. Various distributions can be used to model the noise, however, due to the Central Limit Theorem, section A.4.2, noise with a Gaus- 
sian distribution is used most often. A noise sequence of this type is called a white Gaussian random sequence. Similarly, white noise can be defined for the continuous parameter case. Again, all the $X_{t}$ 's are mutually independent, and the white process $X_{t \in \mathcal{T}}$ is formally defined as:

$$
\mathcal{P}\left(X_{t} / X_{\tau}\right)=\mathcal{P}\left(X_{t}\right) \quad(t>\tau \in \mathcal{T})
$$

\section{A.3.3 Markov Processes}

A Markov process is defined as any discrete or continuous parameter stochastic process that satisfies

$$
\mathcal{P}\left\{x_{t_{n}} \leq \lambda / x_{t_{1}}, \ldots, x_{t_{n-1}}\right\}=\mathcal{P}\left\{x_{t_{n}} \leq \lambda / x_{t_{n-1}}\right\}
$$

where $\lambda$ is a real number, and $\mathcal{T}$ is a parameter set such that $\left\{t_{i}: t_{i} \leq t_{i+1}\right\} \in \mathcal{T}$. Basically, the Markov property stated above in Eqn A.46 says that the probability of transition from the current state of the system to a state in the future is independent of how the system arrived in the current state. The assumption that systems are Markovian will be made often throughout this thesis. The Markov property will be used most often in terms of density functions. Given $t_{1} \leq t_{2} \leq \cdots \leq t_{n}$, the Markovian property is written

$$
p\left(x_{t_{n}} / x_{t_{1}}, \ldots, x_{t_{n-1}}\right)=p\left(x_{t_{n}} / x_{t_{n-1}}\right)
$$


Using the conditional density function definition in conjunction with the Markov property:

$$
\begin{aligned}
p\left(x_{t_{n}}, \ldots, x_{t_{1}}\right) & =p\left(x_{t_{n}} / x_{t_{n-1}}, \ldots, x_{t_{1}}\right) p\left(x_{t_{n-1}}, \ldots, x_{t_{1}}\right) \\
& =p\left(x_{t_{n}} / x_{t_{n-1}}\right) p\left(x_{t_{n-1}}, \ldots, x_{t_{1}}\right) \\
& =p\left(x_{t_{n}} / x_{t_{n-1}}\right) p\left(x_{t_{n-1}} / x_{t_{n-2}}\right) p\left(x_{t_{n-2}}, \ldots, x_{t_{1}}\right) \\
& =p\left(x_{t_{n}} / x_{t_{n-1}}\right) p\left(x_{t_{n-1}} / x_{t_{n-2}}\right) \cdots p\left(x_{t_{2}} / x_{t_{1}}\right) p\left(x_{t_{1}}\right)
\end{aligned}
$$

therefore, the joint density function can be completely determined from an initial density, $p\left(x_{t_{1}}\right.$, and the transitional probability densities, $p\left(x_{t} / x_{\tau}\right)$, for all $\{t>\tau\} \in \mathcal{T}$.

The famous Chapman-Kolmogorov Eqn A.49 also follows from the Markov property. Starting with the marginal density for $p\left(x_{n} / x_{n-2}\right)$ and using the Markov property on the integrand:

$$
\begin{aligned}
p\left(x_{n} / x_{n-2}\right) & =\int p\left(x_{n}, x_{n-1} / x_{n-2}\right) d x_{n-1} \\
& =\int p\left(x_{n} / x_{n-1}, x_{n-2}\right) p\left(x_{n-1} / x_{n-2}\right) d x_{n-1} \\
& =\int p\left(x_{n} / x_{n-1}\right) p\left(x_{n-1} / x_{n-2}\right) d x_{n-1}
\end{aligned}
$$

More generally, by replacing $x_{n-2}$ by $x_{m}, m \leq n-2$,

$$
p\left(x_{n} / x_{m}\right)=\int p\left(x_{n} / x_{n-1}\right) p\left(x_{n-1} / x_{m}\right) d x_{n-1}
$$


Next, by taking the expectation,

$$
\begin{aligned}
E\left[p\left(x_{n} / x_{m}\right)\right] & =E\left[\int p\left(x_{n} / x_{n-1}\right) p\left(x_{n-1} / x_{m}\right) d x_{n-1}\right] \\
& =\iint p\left(x_{n} / x_{n-1}\right) p\left(x_{n-1} / x_{m}\right) p\left(x_{m}\right) d x_{n-1} d x_{m} \\
& =\int p\left(x_{n} / x_{n-1}\right) p\left(x_{n-1}\right) d x_{n-1} . \\
& =p\left(x_{n}\right)
\end{aligned}
$$

Thus, given the initial condition of a system, its density $x_{0}$, the probability density of $x_{n}$ can be determined via the Chapman-Kolmogorov equation.

\section{A.4 Basic Statistical Properties of Random Vari- ables}

\section{A.4.1 The Strong Law of Large Numbers}

From a statistical approach, laws of large numbers suggest that the average of a set of random samples from a large population is likely to be similar to the mean of the whole population. In a probabilistic context, the Strong Law of Large numbers is as follows: Given a sequence of $n$ random variates, $X_{1}, X_{2}, \ldots, X_{n}$, each with a finite expectation in a probability space, the Strong Law of Large Numbers is satisfied if:

$$
\frac{1}{n} \sum_{i=1}^{n}\left(X_{i}-E\left[X_{i}\right]\right) \stackrel{\text { a.s. }}{\longrightarrow} 0
$$

where a.s. means converges almost surely, i.e., with probability one. If the random variates are identically distributed, and all have an expectation $\mu$, the Strong Law of 


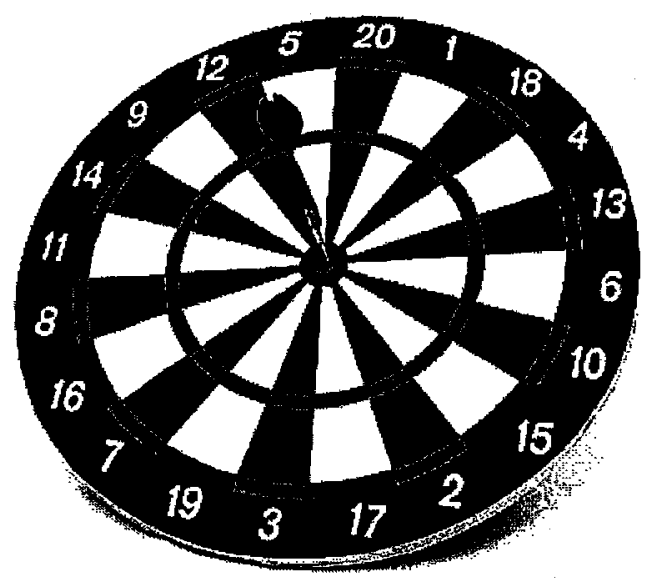

Figure A.4: Almost Surely - The probability of hitting any particular point on a dartboard with a dart is zero.

Large Numbers becomes:

$$
\frac{1}{n} \sum_{i=1}^{n} x_{k} \stackrel{a . s .}{\longrightarrow} \mu \text {. }
$$

Although misleading at first, the term almost surely can be clarified with a simple example. For instance, imagine a square dart board with unit area. The probability of a dart landing in any particular subregion of the dart board is the area of that subregion. The diagonal of the dart board is a particular subregion that has area equal to zero, so the probability of the dart landing on the diagonal is zero. However, the diagonal is not in the empty set, the dart is equally likely to land on any point on the dart board diagonal or not. Thus it can be said a dart thrown at the board will almost surely not land on the diagonal.

\section{A.4.2 The Central Limit Theorem}

According to Ross, the Central Limit Theorem challenges the Strong Law of Large Numbers for the honor of being probability theory's number one result [60]. Given 
a sequence of independent, identically distributed random variables, $X_{1}, X_{2}, \ldots$, each with mean $\mu$ and variance $\sigma^{2}$, the Central Limit Theorem is that the distribution of

$$
\frac{X_{1}+X_{2}+\cdots+X_{n}+n \mu}{\sigma \sqrt{n}}
$$

tends to the standard normal as $n \rightarrow \infty$. That is:

$$
P\left\{\frac{X_{1}+X_{2}+\cdots+X_{n}+n \mu}{\sigma \sqrt{n}} \leq a\right\} \rightarrow \frac{1}{\sqrt{2 \pi}} \int_{-\infty}^{a} e^{-x^{2} / 2} d x
$$

as $n \rightarrow \infty$.

Using white Gaussian noise to model system noise becomes clear with help from the Central Limit Theorem. Assuming the system noise is due to various sources impinging on a measuring device, the Central Limit Theorem implies that their superposition will be somewhat Gaussian. 


\section{Appendix B}

\section{Generating Random Numbers}

\section{B.1 Introduction}

When conducting computer simulations random numbers (RN) are necessary in order to generate synthetic data. RNs are also one of the main elements of particle filters. Particle filters are not possible wihtout efficient random number generation. Similarly, most simulation software uses RNs. Programing languages often have built in routines to generate RNs. For instance, Matlab has the rand() function to produce random numbers according to a uniform distribution on the interval $(0,1)$. This chapter introduces the main concepts in generating random numbers with a digital computer system.

\section{B.2 Properties of Random Numbers}

There are two statistical properties that a sequence of $\mathrm{RNs} R_{1}, R_{2}, R_{3}, \ldots$ must have: uniformity and independence[6]. Consider a uniform distribution on $(0,1)$. Uniformity implies that if the interval $(0,1)$ is divided into $n$ subintervals of length $1 / n$ 
then the expected number of observations in each interval is $N / n$ where $N$ is the total number of observations. The independence property means that the probability of observing a value in a particular subinterval in independent of all the previous observations.

Ideally RNs generated by computer algorithms would satisfy both of the uniformity and independence statistical properties. In reality, however, this is not the case. The next sections describe in detail how the built in Matlab routines generate RNs. Furthermore, how to generate random variates using the built in Matlab routines is also discussed.

\section{B.3 Key Issues in Generating Pseudo-Random Numbers}

As mentioned earlier, it is impossible to generate a truly random sequence. Thus, the goal of pseudo-random number generators (PRNG) is to create sequences of RNs that simulate the ideal properties of uniformity and independence as closely as possible. Although a sequence of pseudo-random numbers (PRN)s may look random they are completely deterministic. In fact, the sequences of PRNs are generated by mathematical formulas. Conversely, a sequence of PRNs that looks random may not mimic the uniformity and independence properties very well. There are many tests that can be used to determine how well a PRNG works. Some of the common tests are frequency tests, runs tests, autocorrelation tests, gap tests, and the Poker test[6]. Similarly, there are many techniques to generate PRNs. The key issues every PRNG should address include:

- Speed - PRNG routines have to be quick. Many simulations require millions of 
random numbers or more.

- Portability - The PRNG should work on many different computer architectures.

- Cycle Period - PRNGs have what is known as a period, that is, the numbers they generate cycle. The cycle period should be sufficient for the application. For example, if your application requires 100 random numbers then the period of the PRNG should be at least greater than 100. A long period is a valuable attribute for PRNGs to have.

- Replicability - When running simulations using sequences of random numbers that can be reproduced is valuable for debugging purposes and comparisons. Similarly, different starting points for the sequence are also desirable.

- Uniformity and Independence - The sequence of PRNs should closely approximate the statistical properties of ideal $\mathrm{RN}$ sequences.

\section{B.4 Generating Uniformly Distributed Pseudo- Random Numbers}

\section{B.4.1 The Linear Congruential Method}

The Linear Congruential Method (LGM) is the the most widely used random number generator [6]. It was initially described by Lehmer in 1951 [47]. It is used to generate a pseudo-random sequence of integers $R_{1}, R_{2}, R_{3}, \ldots$ on the interval $(0, m-1)$. The main relationship of the LGM is

$$
R_{i+1}=\left(a R_{i}+c\right) \bmod m, i=0,1,2, \ldots
$$


where $a$ is called the constant multiplier, $c$ is known as the increment, and finally, $m$ is the modulus. To start generating a sequence of PRNs, the seed $R_{0}$ must be chosen. Seeds are used in many PRNGs. They can be very useful when conducting experiments. For instance, to generate the same sequence of RNs in order to compare results of multiple experiemnts, the same seeds can be used in each simulation. The seed affectively defines a starting point for the $\mathrm{RN}$ sequence and since the generators are deterministic, the sequence will always be that same for a particular seed.

Selecting the values $a, c$, and $m$ affects the statistical properties and cycle length of the sequence. The maximum period possible for the LGM is $2^{b}$. This occurs when $m=2^{b}, c \neq 0$ and is relatively prime to $m$ (i.e., the greatest common factor of $c$ and $m$ is 0$)$, and $a=1+4 k$ where $k$ is an integer.

The following example illustrates how the LGM works. It is from [6]. The variables are set such that $R_{0}=27, a=17, c=43$, and $m=100$. Thus, in this example integer values between 0 and 99 will be produced. Random numbers uniformly distributed between 0 and 1 can be generated using the additional formula

$$
R_{i}^{*}=\frac{R_{i}}{m}, i=1,2,3, \ldots
$$

The algorithm works as follows:

- $R_{0}=27$

- $R_{1}=(17 \cdot 27+43) \bmod 100=2$

$$
R_{1}^{*}=\frac{2}{100}=0.02
$$

- $R_{2}=(17 \cdot 2+43) \bmod 100=77$

$$
R_{2}^{*}=\frac{77}{100}=0.77
$$

- $R_{3}=(17 \cdot 777+43) \bmod 100=52$ 


$$
R_{3}^{*}=\frac{52}{100}=0.52
$$

\section{B.4.2 The Lagged Fibonacci Algorithm with the Shift Reg- ister Random Integer Scheme}

Before Matlab 5, the uniform RNG was a conguential generator with parmarters: $a=7^{5}, c=0$, and $m=2^{31}-1$. Hence the period was just over 2 billion numbers. With the computational power available with the new hardware recetly developed, however, demand for a RNG with a larger period led MathWorks to switch to a RNG with a larger period. The new algorthim is based on George Marsaglia's work [51]. It does not use the congruential method. It is designed specifically to generate floating point numbers rather than integers. The rest of this section is dedicated to explaning how the algorithm works. For more details please see chapter 9 of [55]. The following description is based on that book chapter.

Rather than using an initial seed, the lagged Fibonacci generator has an initial state that is composed of 35 words of memory. Of the 35 words, 32 make up a cache of floating point numbers, $\mathrm{z}$, between 0 and 1 . The rest of the state is composed of two integers, $i$ and $j$, and a borrow flag $b$. The integer $i$ is restrained to be from 0 to 31. The main equation of the generator is

$$
z_{i}=z_{i+20}-z_{i+5}-b
$$

such that the $i^{\text {th }}$ floating point number is the difference of two others. The indices $i$, $i+20$, and $i+5$ are interpreted as $\bmod 32$. If $z_{i}$ is computed to be positive, $b$ is set to zero for the next step. Alternatively, if $z_{i}$ is found to be negative it is made positive 
by adding 1 to it. It this case $b$ is set to $2^{-53}$ for the next step.

As described, the aforementioned RNG has a period of approximately $2^{1430}$. Another bitwise logical operation is applied to increase the period further. The floating point fraction of $z_{i}$ is XORed with the value $j$ in the state before $z_{i}$ is returned. This makes it theoretically possible to generate any floating point number between $2^{-53}$ and $1-2^{-53}$. Additionally, this bit fiddling set increases the period of the RNG to approximately $2^{1492}$ (which leads some people to refer to it as the Christopher Columbus Generator).

\section{B.5 Random Variate Generation}

\section{B.5.1 The Ziggurat Method: Gaussian Distribution}

The Ziggurat method is a sophisticated table lookup routine developed by Marsaglia and Tsang [50]. It is named after the ancient Mesopotamian terraced temple mounds that resemble two-dimensional step functions. There are two main components of the Ziggurat algorithm: initialization and generation.

The initialization stage is used to approximate the pdf of the Gaussian distribution with $N$ horrizontal rectangles, each with equal areas. This stage only needs to be completed once. An illustation is shown in Fig B.1. There are two sets of data that need to be calculated and kept in a table. The first is the set of the right-hand edges of the rectangles, indicated by circles in Fig B.1. The second is the set of $\sigma$ 's that represent fractional areas calculated $\sigma_{k}=z_{k-1} / z_{k}$. Once the data for the table is calculated the rountine to calculate Gaussian random numbers is computationally simple. First a random integer $J$ between 1 and $N$ is calculated. Also, a uniform random number $U$ between -1 and 1 is calculated. A test to see if $U$ lies in the core of the $J^{\text {th }}$ section is conducted, $\left(a b s(U)<\sigma_{J}\right)$. If $U$ does lie in the core then return 


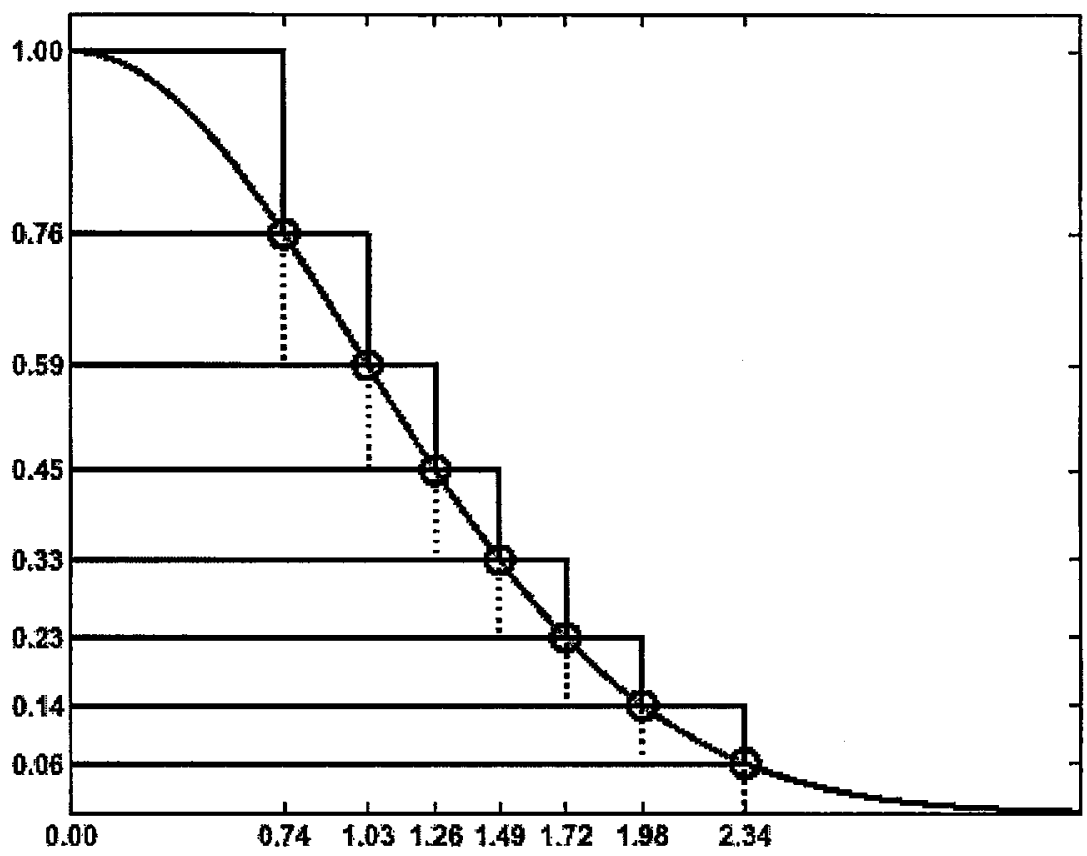

Figure B.1: An illustration of the Ziggurat method with $N=8$. The cirlces represent the $z$ points. (Image from: http://www.mathworks.com/moler/random.pdf [55])

$U z_{J}$

This method returns Gaussian distributed random numbers 97 of the time when $N=128$ [55]. Computationally, the Ziggurat method provided by Maltab randn() is equivallent to the uniform random number generator provided by Matlab rand().

\section{B.5.2 The Acceptance-Rejection Technique: Gamma Distri- bution}

The idea of the Acceptance-Rejection technique is to generate a $\mathrm{RN}$ and then either accept or reject it based on some condition. The acception/rejection step helps make the random variate follow a specific distribution. The efficiency of this algorithm depends on how many rejections are made. The steps to generate a Gamma random variate, $X \sim G a(\alpha, \beta)$, are as follows [6]: 
- Compute $a=(2 \alpha-1)^{\frac{1}{2}}, b=2 \alpha-\ln (4)+\frac{1}{a}$

- Generate RNs $R_{1}$ and $R_{2}$

- Compute $X=\alpha\left(\frac{R_{1}}{1-R_{1}}\right)^{2}$

- If $X>b-\ln \left(R_{1}^{2} R_{2}\right)$ reject $\mathrm{X}$, else use $\mathrm{X}$ as variate

- Set $X=\frac{X}{\alpha \beta}$

\section{B.5.3 Inverse Transform Technique: Cauchy Distribution}

The Inverse Transform technique is a relavtively straight forward method that can be applied to any random distribution that has a closed form inverse CDF. Examples of such distrbutions include exponential, Weibull, and triangular distributions. Examples of distributions who do not include the Gaussian and Gamma distribution. The idea of the Inverse Transform method is to set a variable $R$ equal to the CDF of the random variable, i.e., $R=F(X)$. The to solve the equation in terms of $\mathrm{R}$ such that $X=F^{-1}(R)$. By drawing random numbers $R_{1}, R_{2}, R_{3} \ldots$ the random variates, $X_{i}$, can then be calculated as

$$
X_{i}=F^{-1}\left(R_{i}\right)
$$

In terms of the Cauchy distribution, the CDF is calcutated as

$$
F(x)=\frac{1}{2}+\frac{\arctan \left(\frac{x-x_{0}}{\gamma}\right)}{\pi} .
$$

Hence, using the Inverse Transform method the random variates are

$$
X_{i}=x_{0}+\gamma \tan \left[\pi\left(R_{i}+\frac{1}{2}\right)\right]
$$

